\title{
Localization for equivariant cohomology with varying polarization
}

\author{
Megumi Harada And Yael Karshon \\ Dedicated to the memory of Hans Duistermaat
}

\begin{abstract}
The main contribution of this paper is a generalization of several previous localization theories in equivariant symplectic geometry, including the classical Atiyah-Bott/Berline-Vergne localization theorem, as well as many cases of the localization via the norm-square of the momentum map as initiated and developed by Witten, Paradan, and Woodward. Our version unifies and generalizes these theories by using noncompact cobordisms as in previous work of Guillemin, Ginzburg, and Karshon, and by introducing a more flexible notion of "polarization" than in previous theories. Our localization formulae are also valid for closed 2-forms $\omega$ that may be degenerate. As a corollary, we are able to answer a question posed some time ago by Shlomo Sternberg concerning the classical Brianchon-Gram polytope decomposition,. We illustrate our theory using concrete examples motivated by our answer to Sternberg's question.
\end{abstract}

2 Taming maps and Hamiltonian spaces

2.1 Taming maps and $G$-manifolds

2.2 Taming maps on Hamiltonian $G$-manifolds 
5 Localization formulae for twisted DH distributions

6 The Brianchon-Gram polytope decomposition and symplectic toric manifolds

7 Example: a circle action on the 2-sphere

7.1 Example: a constant taming map

7.2 Example: the norm-square of the momentum map

7.3 Example: the negative of the norm-square of the momentum map

Acknowledgments

Appendix A. Construction on a simple polytope of a smooth function with a unique critical point on the relative interior of each face

References

\section{Introduction}

The main results of this manuscript, Theorems 4.24 and 5.20, are part of a long chain of localization results in topology. Here, by a localization result we mean a formula that expresses a global topological or geometric quantity on a manifold $M$ as a sum of local contributions near a subset of $M$ such as the fixed point set of a torus action or of a diffeomorphism, the zero set of a vector field, or the critical set of a function. This idea has a long history; early results of this general nature are, for instance, the Poincaré-Hopf index theorem, the Lefschetz fixed point theorem, and the early works $[5,6]$ of Bott. Other results that relate to our current work are $[2-4,13,14,16,18$, $24,29,33,34]$. To place our results in the appropriate context, below we give a very brief sketch of this circle of ideas, focusing on aspects that directly relate to our results.

We begin by discussing the classical Duistermaat-Heckman (DH) exact stationary phase formula, which is probably the first such localization result 
in modern symplectic geometry. We will go back and forth between two different perspectives that are frequently encountered in the literature and which are related by a Fourier transform. Let $(M, \omega)$ be a compact symplectic manifold, equipped with an action of a torus $G$ with associated momentum map $\Phi: M \rightarrow \mathfrak{g}^{*}$. In their original papers $[13,14]$, Duistermaat and Heckman consider the oscillatory integral

$$
\int_{M} \frac{\omega^{n}}{n !} \mathrm{e}^{\mathrm{i}\langle\Phi, X\rangle}
$$

over $M$ of the function $\mathrm{e}^{\mathrm{i}\langle\Phi, X\rangle}$ times the Liouville measure, where $\langle\Phi, X\rangle$ denotes the component of the momentum map for $X \in \mathfrak{g}$. They then prove an exact stationary phase formula, which expresses this integral as the sum of local contributions near the fixed points of the $G$-action.

Recall that Liouville measure on $M$ is obtained by integration of $\omega^{n} / n$ !, the symplectic volume form. The DH measure on $\mathfrak{g}^{*}$, which we denote $\mathrm{DH}_{(\mathrm{M}, \omega, \Phi)}$, is the push-forward of Liouville measure via the momentum map $\Phi: M \rightarrow \mathfrak{g}^{*}$. The integral (1.1) is essentially the Fourier transform, denoted $\widehat{\mathrm{DH}}_{(M, \omega, \Phi)}$, of the DH measure. This can be seen from the following computation: for $X \in \mathfrak{g}$ :

$$
\begin{aligned}
\widehat{\mathrm{DH}}_{(M, \omega, \Phi)}(-X) & =\int_{\xi \in \mathfrak{g}^{*}} \mathrm{DH}_{(\mathrm{M}, \omega, \Phi)} \mathrm{e}^{\mathrm{i}\langle\xi, \mathrm{X}\rangle} \\
& =\int_{M} \frac{\omega^{n}}{n !} \mathrm{e}^{\mathrm{i}\langle\Phi, X\rangle},
\end{aligned}
$$

where the first equality follows from the definition of Fourier transform and the second equality follows from the definition of the DH measure.

The integral (1.1) can also be interpreted as a push-forward in equivariant cohomology. With this interpretation, the exact stationary phase formula becomes a special case of a localization formula in equivariant cohomology. This important formula was observed by Berline and Vergne and by Atiyah and Bott, and hence it is often referred to as the "ABBV formula". For details see [2-4].

It is possible to relax some of the assumptions on the manifold $M$ and the 2-form $\omega$ and still have versions of the exact stationary phase formula, as we now describe. One such relaxation is to allow the closed 2-form $\omega$ to be degenerate. The manifold $M$ must then be equipped with an orientation, so that we can still integrate the symplectic volume form. If $M$ is compact then the DH measure remains well defined as a signed measure. The exact 
stationary phase formula continues to hold in this generality; indeed, it still follows from the ABBV formula.

If the manifold $M$ is not compact, but the momentum map $\Phi$ is proper, then the DH measure can be defined as a distribution, even if $\omega$ is degenerate. In this paper we mainly work with this DH distribution (see Definition 4.1). In this situation, there might not exist a localization formula to the fixed point set; there might not even be any fixed points. (This can be seen, for instance, from the example of the action of a torus $G$ on its cotangent bundle $T^{*} G \cong G \times \mathfrak{g}^{*}$ with the momentum map being the projection to $\mathfrak{g}^{*}$.) However, if we additionally assume that $\Phi$ has a component that is proper and bounded from below, then there does exist a localization formula which expresses the DH distribution as a sum of contributions given in terms of infinitesimal data along the components of the $G$-fixed point set. Guillemin, Lerman, and Sternberg derived a formula of this form for the DH measure when $M$ is compact and $G$ is a torus acting with isolated fixed points [18]. Their proof uses an (inverse) Fourier transform applied to the left and righthand sides of the original DH formula for (1.1). The case of non-isolated fixed points is worked out by Guillemin and Cannas da Silva in [11]; the case that a component of the momentum map is proper and bounded from below is analyzed by Prato and $\mathrm{Wu}[30]$.

With these historical remarks in hand, we can describe the motivations and main contributions of the present manuscript. One of our major motivations was to further develop the point of view (initiated in $[15,25]$ and developed in [16]) that it is possible to derive the above-mentioned localization formulae $[11,18]$ directly, without passing through Fourier transforms. The main technique for doing so is an appropriate notion of noncompact cobordism, under the hypothesis that a component of the momentum map is proper and bounded below. Indeed, using cobordisms, one can prove a Guillemin-Lerman-Sternberg-type formula in the more general situation of non-isolated fixed points and non-compact $M$ ([25, Section 11], [16, Chap. 4, Section 6]).

Here we take a moment to sketch some of the history of cobordisms in symplectic geometry. The idea originates from the work of Guillemin and Sternberg, who observe in [21] that two compact Hamiltonian symplectic manifolds have the same DH measure if they are Hamiltonianly cobordant through a compact manifold $W$. This is a simple consequence of Stokes' theorem. Shaun Martin used cobordisms in the context of equivariant localization in [28]. Guillemin, Ginzburg, and the second author, in [16, 25], allow for non-compact Hamiltonian manifolds and use noncompact cobordisms to derive localization results. In this setting, to deal with the lack of 
compactness, one restricts attention to momentum maps that are proper. Moreover, one often makes the stricter requirement that the momentum map is "polarized", i.e., it has a component that is proper and bounded from below. This important technical condition on momentum maps first appeared in the work of Prato and $\mathrm{Wu}[30]$.

Our work generalizes and extends the cobordism ideas in [16] by introducing the notions of a taming map and a $v$-polarized momentum map, as we now explain. The basic idea is that we allow the component $v$ of the momentum map $\Phi$ - with respect to which the function $\langle\Phi, v\rangle$ must be proper and bounded from below (i.e., "polarized") — to vary along the manifold. More precisely, suppose $M$ is a $G$-manifold. We fix a taming map $v: M \rightarrow \mathfrak{g}$, so named because we use it to control situations when the manifold is not compact. Then we require the function obtained by pairing the $\mathfrak{g}^{*}$-valued momentum map with this taming map to be proper and bounded from below, giving us the notion of a $v$-polarized momentum map. In the special case that $v \equiv \eta \in \mathfrak{g}$ is constant, we recover the $\eta$-polarized condition of [16]. Our notion of a taming map is motivated by work of Maxim Braverman [7], although our assumptions on the map $v$ slightly differ from his.

We now describe in more detail the main results of this paper. Let $G$ be a compact Lie group, $M$ an even-dimensional oriented $G$-manifold, $\omega$ a $G$-invariant closed 2-form (not necessarily symplectic), and $\Phi: M \rightarrow \mathfrak{g}^{*}$ a $G$-equivariant function such that Hamilton's equation

$$
d \Phi^{X}=\iota\left(X^{\sharp}\right) \omega
$$

holds for all $X \in \mathfrak{g}$. (We call such a triple a Hamiltonian $G$-manifold, although - contrary to the standard use of the term in the literature - we do not require $(M, \omega)$ to be symplectic.) A taming map $v$ determines a vector field $v^{\sharp}$ on $M$ via the infinitesimal action of the Lie algebra $\mathfrak{g}$ on $M$. The localizing set in our theory is the zero set $Z:=\left\{v^{\sharp}=0\right\} \subseteq M$ of this vector field. The main results of our manuscript, Theorems 4.24 and 5.20, express the DH distribution of the Hamiltonian $G$-manifold $(M, \Phi, \omega)$ (respectively twisted DH distribution) in terms of local data on arbitrarily small neighborhoods of $Z$. Namely, let $Z=\bigcup_{i \in \mathcal{I}} Z_{i}$ be the decomposition of $Z$ into its connected components. Then our Theorem 4.24 takes the form

$$
\mathrm{DH}_{(\mathrm{M}, \Phi, \omega)}=\sum_{\mathrm{i}} \mathrm{DH}_{\mathrm{germ}_{\mathrm{Z}_{\mathrm{i}}}(\mathrm{M}, \omega, \Phi)}^{\mathrm{v}}
$$


and, in the twisted case, our Theorem 5.20 takes the form

$$
\mathrm{DH}_{(\mathrm{M}, \omega, \Phi)}(\mathrm{A})=\sum_{\mathrm{i}} \mathrm{DH}_{\mathrm{germ}_{\mathrm{Z}_{\mathrm{i}}}(\mathrm{M}, \omega, \Phi)}^{\mathrm{v}}\left(\left.\mathrm{A}\right|_{\mathrm{Z}_{\mathrm{i}}}\right)
$$

where $A$ is an equivariant cohomology class on $M$. The $i$ th summand on the right-hand side is defined to be the $\mathrm{DH}$ distribution (respectively twisted $\mathrm{DH}$ distribution) of a $v$-polarized completion relative to $Z_{i}$ (made precise in Definition 3.3) of the restriction of $(M, \omega, \Phi)$ to an arbitrarily small $G$-invariant neighborhood of the connected component $Z_{i}$ of $Z$. The nota-

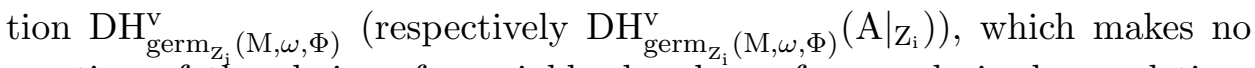
mention of the choice of a neighborhood or of a $v$-polarized completion, is justified since we prove in Section 4 (respectively Section 5) that under appropriate hypotheses this DH distribution (respectively twisted DH distribution) is in fact independent of these choices. In this sense the right-hand sides of (1.3) and (1.4) depend only on data that is localized near $Z$, and thus it is valid to view our results as a "localization to $Z$ ". We emphasize that the specific form of the right-hand side varies according to choice of $v$ (and hence $Z$ ), so actually each of (1.3) and (1.4) is a family of formulae; we explicitly demonstrate this using a simple example in Section 7.

Next we relate our main results to another related circle of ideas, namely that of the so-called nonabelian localization and localization with respect to the norm-square of the momentum map. We begin with a brief historical account. The "nonabelian localization" theory was initiated by Witten, who considers integrals of the form

$$
\int_{\mathfrak{g}} \mathrm{e}^{-\varepsilon\|X\|^{2}} \int_{M} \eta(X) \mathrm{e}^{\omega+\mathrm{i} \Phi} d X
$$

where $\eta$ is an equivariant differential form, $d X$ is a volume form on $\mathfrak{g}$, the $\varepsilon$ is a real parameter, and $\Phi$ again denotes the momentum map for a Hamiltonian $G$-action (see [33, p. 311] and [27, Section 2]). The group $G$ may be nonabelian. The integral (1.5) can be interpreted as the evaluation on the Gaussian $\mathrm{e}^{-\varepsilon\|X\|^{2}}$ of a twisted $\mathrm{DH}$ distribution $\widehat{\mathrm{DH}}_{(M, \omega, \Phi)}(\eta)$ on $\mathfrak{g}$. (Mild assumptions on $M$ guarantee that this distribution is a temperate (a.k.a tempered) distribution, so its evaluation on the Gaussian is well defined.) Witten then gives a formula similar in spirit to the abelian ABBV localization formula mentioned above; he expresses (1.5) as a sum of local contributions from the components of the critical set of $\|\Phi\|^{2}$, where the dominant contribution as $\varepsilon \rightarrow \infty$ is from the absolute minimum $\Phi^{-1}(0)$. (In fact Witten's theory is slightly more general: he begins with a localization formula 
which depends on a choice of closed invariant 1-form $\lambda$ on $M$ and leads to a sum of local contributions from the components of the set

$$
\left\{x \mid\left\langle\lambda, \xi^{\sharp}\right\rangle=0\right\}
$$

for appropriate vector fields $\xi^{\sharp}$. He then specializes to the case $\lambda=d\|\Phi\|^{2}$, for which the set (1.6) is Crit $\|\Phi\|^{2}$.)

Witten's results may be considered an extension of the abelian DH theory, in that it firstly introduces more general integrands (which correspond to the twisted DH distributions of Section 5), and secondly it produces formulae that localize to $\operatorname{Crit}\left(\|\Phi\|^{2}\right)$, the components of the critical set of the norm-square of $\Phi$, instead of the fixed points of the action. Some years later, Jeffrey and Kirwan derived similar formulae by working through the maximal torus $T$ of a compact nonabelian Lie group $G$; they also explain the relation of their formula to that of Witten's in [24]. Kefeng Liu gives simplified proofs of some of these results in [27]. More recently, Paradan [29] and Woodward [34] develop a localization theory for the norm-square $\|\Phi\|^{2}$ of the momentum map which incorporates the Witten integrals above, deriving formulae for (the Fourier transforms of) the twisted DH distributions as sums of local contributions associated to components of the critical set of $\|\Phi\|^{2}$.

Our main theorems also generalize (the inverse Fourier transforms of) the nonabelian localization formulae to $\operatorname{Crit}\left(\|\Phi\|^{2}\right)$, under the assumption that the connected components of $\operatorname{Crit}\left(\|\Phi\|^{2}\right)$ are smooth. (We expect this assumption to not be necessary; see Remark 4.23.) Namely, we express the (twisted) DH distribution as a sum of local contributions. Our formulae rely on a choice of taming map $v: M \rightarrow \mathfrak{g}$, which can be obtained from a choice of a real valued function $\rho: \mathfrak{g}^{*} \rightarrow \mathbb{R}$ by $(2.20)$. The localizing set is then the critical set of the composition $\rho \circ \Phi$. When $G$ is a torus and $\rho$ is a linear functional, as described in our Example 2.26, our Theorem 4.24 recovers the so-called GLS formula [18]. When $\rho$ is the norm square function $\|\cdot\|^{2}: \mathfrak{g}^{*} \rightarrow \mathbb{R}$, as described in our in Example 2.27, we recover the localizing set $\operatorname{Crit}\left(\|\Phi\|^{2}\right)$ of Witten, Paradan, and Woodward [29, 33, 34].

Our results also answer a question asked some time ago by Shlomo Sternberg concerning the Brianchon-Gram polytope decomposition. It is known that the Atiyah-Bott-Berline-Verge localization theorem in equivariant cohomology [2-4], when applied to the exponent of the equivariant symplectic form of a compact symplectic toric manifold, yields the measuretheoretic version of the Lawrence-Varchenko polytope decomposition [26, $32]$, when applied to the corresponding momentum polytope. Sternberg had 
asked whether there is a similar "localization-theoretic" interpretation of the classical Brianchon-Gram polytope decomposition. We can answer Sternberg's question in the affirmative: a special case of Theorem 4.24, applied to the exponent of the equivariant symplectic form on a compact symplectic toric manifold, yields (the measure-theoretic version of) the BrianchonGram polytope decomposition for the momentum polytope. However, this application requires the fact that, for any simple polytope, there exists a smooth function with a unique critical point on the relative interior of every face of that polytope (and satisfies some additional technical conditions). If the polytope satisfies a technical assumption (recorded in (6.15)), then the norm-square function has this property. Surprisingly, proving that for any simple polytope there exists such a function turned out to be not entirely trivial, and our proof (by brute-force differential topology on $\mathbb{R}^{n}$ ) occupies Appendix 7.3.

In future work, it would be interesting to investigate whether our results generalize to $G$-spaces that are not manifolds. (Such a generalization may correspond to polytope decompositions for non-simple polytopes, such as Haase's generalization of the Lawrence-Varchenko decomposition to nonsimple polytopes [22], or the Brianchon-Gram decomposition applied to non-simple polytopes.) It would also be interesting to see if a combination of our results with Braverman's work in [7] would yield new index formulae.

We now give an outline of the contents of this paper. In Section 2, we define the taming map and the corresponding localizing set and we describe some of our motivating examples. In Section 3 , we define $v$-polarized completions and prove that they exist. We note that polarized completions are used both in the formulation of our localization formulae (1.3) and (1.4) and in their proof. We prove the untwisted version of our localization formula, Theorem 4.24, in Section 4 and the twisted version, Theorem 5.20, in Section 5. In Section 6 we discuss the Brianchon-Gram polytope decomposition and answer Sternberg's question in the affirmative. In Section 7, we use the standard $S^{1}$-action on $S^{2}$ in order to illustrate in detail how our equation (1.3) can yield different localization formulae by choosing different taming maps. We prove a technical lemma required in Section 6 in Appendix 7.3.

\section{Taming maps and Hamiltonian spaces}

The "varying polarization" of our localization formulae is controlled by a so-called taming map. In this section, we introduce the notion of a taming map, its associated vector field, and the corresponding notion of a polarization. For a symplectic manifold with Hamiltonian $G$ action and momentum 
map $\Phi: M \rightarrow \mathfrak{g}^{*}$, we explain that a choice of an invariant smooth function $\rho: \mathfrak{g}^{*} \rightarrow \mathbb{R}$ gives rise to a taming map whose localizing set is the critical set of the composition $\rho \circ \Phi$.

\subsection{Taming maps and $G$-manifolds}

Throughout this manuscript, we use $G$ to denote a compact Lie group, $\mathfrak{g}$ its Lie algebra, $\mathfrak{g}^{*}$ the dual space of $\mathfrak{g}$, and $\langle\cdot, \cdot\rangle: \mathfrak{g}^{*} \times \mathfrak{g} \rightarrow \mathbb{R}$ the natural pairing between a vector space and its dual. We equip $\mathfrak{g}$ with an Ad-invariant inner product and $\mathfrak{g}^{*}$ with the induced inner product. We also fix an $\mathrm{Ad}_{G}$-invariant inner product on the Lie algebra $\mathfrak{g}$, and we denote the resulting isomorphism $\mathfrak{g} \rightarrow \mathfrak{g}^{*}$ by $\xi \mapsto \widehat{\xi}$.

Let $N$ be a manifold, possibly with boundary. ${ }^{1}$ A smooth $G$-action on $N$ is a homomorphism $G \rightarrow \operatorname{Diff}(N)$ that is smooth in the diffeological sense, i.e., the map $(g, x) \mapsto g \cdot x$ is smooth as a map from $G \times N$ to $N$. A manifold $N$ equipped with a $G$-action is called a $G$-manifold. If $N$ has boundary, then its boundary $\partial N$ is a manifold, any diffeomorphism of $N$ restricts to a diffeomorphism of $\partial N$, and a smooth $G$-action on $N$ restricts to a smooth $G$-action on $\partial N$.

The following simple notion will be crucial in what follows.

Definition 2.1. Let $N$ be a $G$-manifold, possibly with boundary. A taming map on $N$ is a smooth $G$-equivariant function $v: N \rightarrow \mathfrak{g}$.

We now associate to a taming map $v: N \rightarrow \mathfrak{g}$ a vector field $v^{\sharp}$ on $N$. Recall that a Lie algebra element $X \in \mathfrak{g}$ gives rise to a vector field $X^{\sharp} \in$ $\operatorname{Vect}(N)$ by

$$
\left.X^{\sharp}\right|_{x}:=\left.\frac{d}{d t}\right|_{t=0}(\exp (t X) \cdot x)
$$

for all $x \in N$. The resulting map

$$
\mathfrak{g} \rightarrow \operatorname{Vect}(N), \quad X \mapsto X^{\sharp},
$$

is $G$-equivariant with respect to the adjoint action of $G$ on $\mathfrak{g}$ and the $G$-action on the space of vector fields $\operatorname{Vect}(N)$ that is induced from the $G$-action on $N$. Also, if $N$ has boundary, then the restriction of the vector field $X^{\sharp}$ to the boundary $\partial N$ is a vector field on $\partial N$. Using this notion, we associate to a

\footnotetext{
${ }^{1}$ In this manuscript, manifolds are $C^{\infty}$-smooth, Hausdorff, and second-countable.
} 
taming map $v: N \rightarrow \mathfrak{g}$ a vector field $v^{\sharp}$ on $N$ by

$$
\left.v^{\sharp}\right|_{x}:=\left.v(x)^{\sharp}\right|_{x} \in T_{x} N
$$

for all $x \in N$.

The zero set of this vector field $v^{\sharp}$ will serve as the localizing set for our theory, in the sense that our localization formulae give expressions for global invariants (namely, DH distributions) in terms of data near the localizing sets:

Definition 2.4. The localizing set associated to the taming map $v: N \rightarrow$ $\mathfrak{g}$ is defined by

$$
Z:=\left\{x \in N\left|v^{\sharp}\right|_{x}=0\right\}
$$

If $N$ is a $G$-manifold with boundary, $v: N \rightarrow \mathfrak{g}$ is a taming map, and $x$ is in the boundary of $N$, then $\left.v^{\sharp}\right|_{x}$ is tangent to the boundary and $\left.\left(v^{\sharp}\right)\right|_{\partial N}=$ $\left(\left.v\right|_{\partial N}\right)^{\sharp}$.

The $G$-equivariance of the taming map implies that the associated vector field $v^{\sharp}$ and localizing sets also behave well with respect to the $G$-action:

Lemma 2.5. Let $N$ be a G-manifold, possibly with boundary, and let $v: N \rightarrow \mathfrak{g}$ be a taming map. Then the associated vector field $v^{\sharp}$ is $G$-equivariant, and the localizing set $Z=\left\{v^{\sharp}=0\right\}$ is $G$-invariant.

Proof. For all $x \in N$,

$$
\begin{aligned}
g_{*}\left(\left.v^{\sharp}\right|_{x}\right) & =\left(\operatorname{Ad}_{g} v(x)\right)_{g \cdot x}^{\sharp} & \text { by } G \text {-equivariance of }(2.2) \\
& =(v(g \cdot x))_{g \cdot x}^{\sharp} & \text { by } G \text {-equivariance of } v: N \rightarrow \mathfrak{g} \\
& =\left.v^{\sharp}\right|_{g \cdot x} & \text { by }(2.3),
\end{aligned}
$$

where $g_{*}: T N \rightarrow T N$ denotes the differential of the diffeomorphism $g: N \rightarrow$ $N$. Hence the vector field $v^{\sharp}$ is $G$-invariant, as desired. The $G$-invariance of $Z$ follows from the equivariance of $v^{\sharp}$.

Remark 2.6. We are not the first to use the term taming map. In [7] Braverman considers a complete Riemannian manifold $N$, equipped with an action of a compact Lie group $G$ by isometries. In this setting, he calls a function $v: N \rightarrow \mathfrak{g}$ a taming map if the zero set of the induced vector field is compact. In [7, Def. 3.2] he requires a cobordism of such structures to be consistent in a suitable sense with a choice of tubular neighborhood of the 
boundary. Our definitions are slightly different from Braverman's in that we do not equip $N$ with a Riemannian metric, and, more significantly, we do not require the localizing set to be compact.

Remark 2.7. The localizing set $Z$ is not necessarily smooth. See Remark 2.32.

As in the book [16], we work with possibly noncompact $G$-manifolds $N$ equipped with maps $\Phi: N \rightarrow \mathfrak{g}^{*}$. We deal with the non-compactness of $N$ by requiring a properness condition:

Definition 2.8. Let $N$ be a $G$-manifold, possibly with boundary, and let $v: N \rightarrow \mathfrak{g}$ be a taming map. We say that a continuous function $\Phi: N \rightarrow \mathfrak{g}^{*}$ is $\boldsymbol{v}$-polarized if the function

$$
\Phi^{v}:=\langle\Phi, v\rangle: N \rightarrow \mathbb{R}
$$

is proper and bounded from below.

Definition 2.8 is well-suited for our purposes for two reasons. First, being $v$-polarized frequently implies that the original map $\Phi$ is proper (see Lemma 2.9 below). Second, being $v$-polarized is preserved under patchings by a partition of unity or averaging with respect to compact group actions (see Section 3).

We make the following purely topological observations:

Lemma 2.9. Let $N$ be a $G$-manifold, possibly with boundary. Let $v: N \rightarrow \mathfrak{g}$ be a taming map and let $\Phi: N \rightarrow \mathfrak{g}^{*}$ be a continuous function.

(1) If $N$ is compact, then $\Phi$ is v-polarized.

(2) Suppose that $\Phi: N \rightarrow \mathfrak{g}^{*}$ is v-polarized. Let $Y$ be a subset of $N$. Then the restriction to $Y$ of $\Phi$ is v-polarized if and only if $Y$ is closed in $N$.

(3) Suppose that $v$ is bounded. Then

$$
\Phi \text { is } v \text {-polarized } \Rightarrow \Phi \text { is proper. }
$$

Proof. Part (1) follows from the fact that every continuous function on a compact set is bounded and proper.

For Part (2) recall that for a proper map $\psi: N \rightarrow \mathbb{R}$ on a Hausdorff space $N$ and a subset $Y$ of $N$, the restriction $\left.\psi\right|_{Y}: Y \rightarrow \mathbb{R}$ is proper if and only if $Y$ is closed. Indeed, if $Y$ is closed then $\psi^{-1}([a, b]) \cap Y$ is compact 
for any interval $[a, b]$. This implies $\left.\psi\right|_{Y}$ is proper. Now suppose that $\left.\Psi\right|_{Y}$ is proper, and let $x$ be an accumulation point of $Y$. Let $a$ and $b$ be such that $a<\psi(x)<b$. Then $x$ is also an accumulation point of $\Psi^{-1}([a, b]) \cap Y$. Because $\left.\psi\right|_{Y}$ is proper, $\Psi^{-1}([a, b]) \cap Y$ is closed. So $x$ is in $Y$. Because $x$ was arbitrary, this shows that $Y$ is closed. Claim (2) follows.

We now prove part (3). Choose an inner product on $\mathfrak{g}$, and consider the induced inner product on $\mathfrak{g}^{*}$. Then for any $x \in N$,

$$
\begin{aligned}
|\langle\Phi(x), v(x)\rangle| & \leq\|\Phi(x)\| \cdot\|v(x)\| \quad \text { by the Cauchy-Schwarz inequality } \\
& \leq c\|\Phi(x)\|
\end{aligned}
$$

where $c:=\sup _{x \in N}\|v(x)\|<\infty$ exists because $v$ is bounded by assumption. Now let $K$ be a compact subset of $\mathfrak{g}^{*}$. By (2.10), $\Phi(x) \in K$ implies that $|\langle\Phi(x), v(x)\rangle| \leq c r$, where $r=\sup _{\alpha \in K}\|\alpha\|$. So the $\Phi$-preimage of $K$ is contained in the $\langle\Phi, v\rangle$-preimage of the interval $[-c r, c r]$, which is compact because $\langle\Phi, v\rangle$ is proper by assumption. Being a closed subset of a compact set, $\Phi^{-1}(K)$ is also compact, as required.

Remark 2.11. The converse of part (3) of Lemma 2.9 is generally false: a proper map to $\mathfrak{g}^{*}$ need not be $v$-polarized, even if $v$ is bounded. For example, the identity map on $N=\mathfrak{g}^{*}$ is proper but is not $v$-polarized if $v: N \rightarrow \mathfrak{g}$ is constant.

Remark 2.12. In Sections 4 and 5, we derive localization formulae that depend on a choice of taming map. However, the role played by this choice is quite loose in the sense that many choices of $v$ give the same localization formulae. Specifically, we may define two taming maps $v_{1}$ and $v_{2}$ on a $G$-manifold $N$ to be equivalent if there exists a $G$-invariant positive function $f: N \rightarrow \mathbb{R}_{>0}$ such that both $f$ and $1 / f$ are bounded and such that $v_{2}=f v_{1}$. If $v_{1}$ and $v_{2}$ are equivalent taming maps then

- they have the same localizing set; (see Definition 2.4);

- a function $\Phi: N \rightarrow \mathfrak{g}^{*}$ is $v_{1}$-polarized if and only if it is $v_{2}$-polarized.

Equivalent bounded taming maps give rise to the same localization formulae in Sections 4 and 5. See Remarks 4.29 and 5.25.

Remark 2.13. We often require taming maps to be bounded, because if $v: N \rightarrow \mathfrak{g}$ is bounded then every $v$-polarized map $N \rightarrow \mathfrak{g}^{*}$ is proper (by 
Part (3) of Lemma 2.9). On the other hand, sometimes it is more natural to begin with a taming map $v: N \rightarrow \mathfrak{g}$ that is unbounded (see Example 2.27). In this situation, we can replace $v$ with a bounded taming map $v^{b}: N \rightarrow \mathfrak{g}^{*}$ by defining

$$
v^{b}(x):=h(\|v(x)\|) v(x),
$$

where $\|\cdot\|$ is an $\operatorname{Ad}_{G}$-invariant norm on $\mathfrak{g}$ and $h: \mathbb{R}_{\geq 0} \rightarrow \mathbb{R}_{\geq 0}$ is a smooth function such that $h(r)=1$ for $r$ near $0, h(r)=1 / r$ for $r \geq 1$, and such that the function $r \mapsto h(r) \cdot r$ is weakly monotone. We can then derive a localization formula using the taming map $v^{b}$. Different choices of the function $h$ result in bounded taming maps $v^{b}$ that are equivalent in the sense of Remark 2.12. Moreover, if $v$ was already bounded, then $v^{b}$ is also equivalent to $v$. In this sense we can get a localization formula from any taming map $v: N \rightarrow \mathfrak{g}$.

Remark 2.14. Braverman works with a similar though not identical freedom in [7]. He needs his map $v: N \rightarrow \mathfrak{g}$ to be sufficiently large in a suitable sense. He achieves this by multiplying $v$ by a real valued function that grows sufficiently fast, but his formulae are independent of the choice of this function.

Remark 2.15. The equivalence relation of Remarks 2.12 and 2.13 is still finer than necessary for our purposes in the sense that many inequivalent taming maps still give rise to the same localization formula. For example, suppose that a torus $T$ acts on a compact symplectic manifold with a finite fixed point set. Let $\eta$ be an element of the Lie algebra of $T$ whose pairings with all the isotropy weights at all the fixed points are nonzero. When the taming map takes the constant value $\eta$, our localization formula, Theorem 4.24, boils down to the Guillemin-Lerman-Sternberg formula [18]. The right-hand side of this formula depends only on the signs of the pairings of $\eta$ with the isotropy weights.

\subsection{Taming maps on Hamiltonian $G$-manifolds}

In this section we focus our attention on Hamiltonian $G$-manifolds, and we discuss some motivating examples. As before, we denote by $\Phi^{X}$ the $X$-component of a function $\Phi: N \rightarrow \mathfrak{g}^{*}$, i.e., $\Phi^{X}(\cdot):=\langle\Phi(\cdot), X\rangle: N \rightarrow \mathbb{R}$ for $X \in \mathfrak{g}$.

We note that in our definition of Hamiltonian $G$-manifold the 2 -form is allowed to be degenerate. 
Definition 2.16. Let $N$ be an oriented $G$-manifold, possibly with boundary. Let $\omega$ be a $G$-invariant closed 2-form, and let $\Phi: N \rightarrow \mathfrak{g}^{*}$ be a $G$-equivariant function such that Hamilton's equation

$$
d \Phi^{X}=\iota\left(X^{\sharp}\right) \omega
$$

holds for all $X \in \mathfrak{g}$. Such a triple $(N, \omega, \Phi)$ is called a Hamiltonian $\boldsymbol{G}$-manifold, and the map $\Phi: N \rightarrow \mathfrak{g}^{*}$ is called a momentum map.

In the symplectic geometry literature, the term "Hamiltonian $G$-manifold" is usually reserved for $G$-actions on symplectic manifolds, i.e., the closed 2-form $\omega$ is additionally required to be nondegenerate. When the form $\omega$ is allowed to be degenerate as in our Definition 2.16, some authors (e.g., Woodward in [34, Section 3.1]) call the structure a "degenerate Hamiltonian G-manifold". As in the book [16] (see, e.g., [16, Chap. 2, Section 1.1]), we deviate slightly from this terminology, for two reasons. First, our localization formulae are also valid for closed 2-forms that are somewhere degenerate. Second, because the derivation of our formulae uses cobordisms, we work with both even- and odd-dimensional manifolds, and a closed 2-form on an odd-dimensional manifold is everywhere degenerate.

Remark 2.18. In Definition 2.16, if $N$ is a manifold with boundary, the restrictions of $\omega$ and $\Phi$ to the boundary also satisfy Hamilton's equations.

Since our definition of Hamiltonian $G$-spaces does not include assumptions of properness of the momentum map nor nondegeneracy of the 2-form, we use the following additional terminology:

Definition 2.19. We say that a Hamiltonian $G$ manifold $(N, \omega, \Phi)$ is proper if the momentum map $\Phi: N \rightarrow \mathfrak{g}^{*}$ is proper; we say that it is nondegenerate if the closed 2 -form $\omega$ is nondegenerate and, unless we say otherwise, the orientation of $N$ is induced from $\omega$.

Given a Hamiltonian $G$-space $N$, the main technical idea of our constructions in the next section is to associate to a momentum map $\Phi: N \rightarrow \mathfrak{g}^{*}$ a taming map $v$ such that $\Phi$ is $v$-polarized, and then to vary the 2 -form and momentum map (on $N$ as well as on an appropriate cobording manifold) while maintaining the taming map that was built from the original momentum map. As a first step we now describe a way to obtain a taming map from a momentum map. 
We can view the differential of a smooth function $\rho: \mathfrak{g}^{*} \rightarrow \mathbb{R}$ as a function $d \rho: \mathfrak{g}^{*} \rightarrow \mathfrak{g}$, since for any $\alpha \in \mathfrak{g}^{*}$ the differential $\left.d \rho\right|_{\alpha}$ at $\alpha$ is an element of $\operatorname{Hom}\left(T_{\alpha} \mathfrak{g}^{*}, \mathbb{R}\right) \cong \mathfrak{g}$. If $\rho$ is $G$-invariant, then $d \rho$ is $G$-equivariant. Thus, given a $G$-invariant smooth function $\rho: \mathfrak{g}^{*} \rightarrow \mathbb{R}$ and a momentum map $\Phi: N \rightarrow \mathfrak{g}^{*}$, we may compose $\Phi$ with the differential $d \rho$ to obtain a taming map

$$
v:=d \rho \circ \Phi: N \rightarrow \mathfrak{g} .
$$

Notice that if $G$ is abelian then the $G$ action on $\mathfrak{g}^{*}$ is trivial so every function $\rho: \mathfrak{g}^{*} \rightarrow \mathbb{R}$ is $G$-invariant. We will need the following two technical lemmas:

Lemma 2.21. Let $(N, \Phi, \omega)$ be a Hamiltonian $G$-manifold and let $\rho: \mathfrak{g}^{*} \rightarrow$ $\mathbb{R}$ be a $G$-invariant smooth function. Let $v=d \rho \circ \Phi$ be the corresponding taming map as in (2.20) and let $v^{\sharp}$ be the associated vector field on $N$. Then

(1) $v^{\sharp}$ satisfies Hamilton's equation for the function $\rho \circ \Phi: N \rightarrow \mathbb{R}$, and

(2) if $\omega$ is non-degenerate, the localizing set coincides with the critical set of the function $\rho \circ \Phi$ :

$$
Z:=\left\{v^{\sharp}=0\right\}=\operatorname{Crit}(\rho \circ \Phi) .
$$

Proof. At each point $x \in N$,

$$
\begin{aligned}
\left.d(\rho \circ \Phi)\right|_{x} & =\left.d \rho\right|_{\Phi(x)}\left(\left.d \Phi\right|_{x}\right) \quad \text { by the chain rule } \\
& =\left\langle v(x),\left.d \Phi\right|_{x}\right\rangle \quad \text { by the definition of } v \\
& =\left.\iota\left(v^{\sharp}\right) \omega\right|_{x} \text { by the definition of } v^{\sharp} \text { and Hamilton's equation for } \Phi .
\end{aligned}
$$

The second assertion follows from the first assertion by the non-degeneracy of $\omega$.

The next lemma describes the localizing set $Z$ in terms of orbit type strata. We will need the following terminology. Let $(N, \omega, \Phi)$ be a nondegenerate Hamiltonian $G$-manifold. Given $x$ in $N$, let $G_{x}$ denote the stabilizer subgroup in $G$ of $x$, let $\mathfrak{g}_{x}$ denote the Lie algebra of $G_{x}$ and let $\mathfrak{g}_{x}^{0}$ denote the annihilator of $\mathfrak{g}_{x}$ in $\mathfrak{g}^{*}$. Since $\omega$ is non-degenerate, Hamilton's equation (2.17) implies that

$$
\text { image }\left.d \Phi\right|_{x}=\mathfrak{g}_{x}^{0} \text {. }
$$


Now suppose that $G$ is a torus and let $S$ denote the orbit type stratum through a point $x \in N$. Then $S$ is the connected component of $x$ in the subset

$$
\left\{x^{\prime} \in N \mid G_{x^{\prime}}=G_{x}\right\}
$$

consisting of points with the same stabilizer as $x$. The image of $S$ under $\Phi$ is an open subset of the affine plane $\Phi(x)+\mathfrak{g}_{x}^{0} \subset \mathfrak{g}^{*}[20]$. (For example, if $N$ is a toric variety, $\Phi(S)$ is the relative interior of a face of the momentum map polytope.) Hence

$$
T_{\Phi(x)} \Phi(S)=\mathfrak{g}_{x}^{0}
$$

Lemma 2.25. Let $G$ be a torus, $(N, \omega, \Phi)$ a non-degenerate Hamiltonian $G$-manifold, $\rho: \mathfrak{g}^{*} \rightarrow \mathbb{R}$ a G-invariant smooth function, $v=d \rho \circ \Phi$ the corresponding taming map as in (2.20), and $Z=\left\{v^{\sharp}=0\right\}$ the corresponding localizing set. Let $x \in N$, and let $S$ be the orbit type stratum that contains $x$. Then

$$
x \in Z \text { if and only if } \Phi(x) \text { is a critical point for }\left.\rho\right|_{\Phi(S)} \text {. }
$$

Proof. We have

$$
\begin{aligned}
v^{\sharp}(x)=0 & \text { if and only if }\left.d(\rho \circ \Phi)\right|_{x}=0 \quad \text { by }(2.22) \\
& \text { if and only if }\left(\left.d \rho\right|_{\Phi(x)}\right)\left(\mathfrak{g}_{x}^{0}\right)=0 \quad \text { by }(2.23) \\
& \text { if and only if } \Phi(x) \text { is a critical point for }\left.\rho\right|_{\Phi(S)} \quad \text { by }(2.24) .
\end{aligned}
$$

The main localization results for Hamiltonian $G$-manifolds that occur in the current literature involve two different localizing sets $Z$ : the critical set for a component of the momentum map, and the critical set for the normsquare of the momentum map. We now show that these localizing sets (and accompanying properness conditions often used in the theory) are special cases of our general construction.

Example 2.26. Let $G$ be a torus. Fix a Lie algebra element $\eta \in \mathfrak{g}$, and consider the corresponding linear functional on the dual space, $\rho(\cdot):=\langle\cdot, \eta\rangle$ : $\mathfrak{g}^{*} \rightarrow \mathbb{R}$. Since $G$ is abelian, the coadjoint $G$-action on $\mathfrak{g}^{*}$ is trivial, so $\rho$ is a $G$-invariant function. Moreover, the differential of $\rho$ is the function $\mathfrak{g}^{*} \rightarrow \mathfrak{g}$ with constant value $\eta$. Then, for a Hamiltonian $G$-manifold $(N, \omega, \Phi)$, the corresponding taming map is the function $v: N \rightarrow \mathfrak{g}$ with constant value 
$\eta$, and the function $\Phi^{v}: N \rightarrow \mathbb{R}$ is just the $\eta$-component of the momentum map $\Phi$. For a generic choice of $\eta \in \mathfrak{g}$, the zero set of the vector-field $v^{\sharp}=\eta^{\sharp}$ coincides with the set of fixed points for the entire torus $G$, so

$$
Z=N^{G}
$$

Thus, we recover the classical localizing set of the original DH theorem. Moreover, for this choice of $v$, the momentum map $\Phi$ is $v$-polarized exactly when its $\eta$-component $\langle\Phi, \eta\rangle$ is proper and bounded below. This important condition in the theory of momentum maps was first introduced and analyzed by Prato and $\mathrm{Wu}$ in [30]. In the book [16], a function $\Phi$ that satisfies this condition with respect to an element $\eta \in \mathfrak{g}$ is said to be $\eta$-polarized.

In the next example we allow $G$ to be non-abelian.

Example 2.27. Let $G$ be a compact Lie group and let $(N, \Phi, \omega)$ be a Hamiltonian $G$-space. Consider the norm-square function on $\mathfrak{g}^{*}$

$$
\rho: \mathfrak{g}^{*} \rightarrow \mathbb{R}, \quad \rho(\xi)=\|\xi\|^{2}
$$

Composing $\Phi$ with the differential of $\rho$, it is straightforward to compute that

$$
v(x):=d \rho \circ \Phi(x)=2 \widehat{\Phi}(x),
$$

where $\widehat{\Phi}(x)$ is the element of $\mathfrak{g}$ that corresponds to the element $\Phi(x)$ of $\mathfrak{g}^{*}$ under the identification $\mathfrak{g} \cong \mathfrak{g}^{*}$. So $\Phi^{v}=\langle\Phi, 2 \widehat{\Phi}\rangle=2\|\Phi\|^{2}$. Thus, when $\omega$ is nondegenerate, our theory recovers the localizing set of Witten [33], Paradan [29], and Woodward [34]:

$$
Z:=\left\{v^{\sharp}=0\right\}=\operatorname{Crit}\left(\|\Phi\|^{2}\right) .
$$

Since the norm-square $\|\Phi\|^{2}$ is proper if and only if $\Phi$ is proper, the momentum map $\Phi$ is $v$-polarized if and only if it is proper. (Contrast with Remark 2.11.)

Remark 2.30. In each of the above examples, the $v$-component of $\Phi$ is in fact a multiple of the Hamiltonian function $\rho \circ \Phi$ : indeed, in Example 2.26, we have $\Phi^{v}=\langle\Phi, \eta\rangle=\rho \circ \Phi$, while in Example 2.27, we have $\Phi^{v}=\langle\Phi, 2 \widehat{\Phi}\rangle=2\|\Phi\|^{2}=2(\rho \circ \Phi)$. These are instances of the following more 
general statement: if $\rho: \mathfrak{g}^{*} \rightarrow \mathbb{R}$ is homogeneous of degree $k$, then

$$
\Phi^{v}=k(\rho \circ \Phi) .
$$

To see this, for given $x \in N$, setting $\alpha=\Phi(x)$,

$$
\Phi^{v}(x)=\langle\Phi(x), v(x)\rangle=\left\langle\Phi(x),\left.d \rho\right|_{\Phi(x)}\right\rangle=\left(L_{\alpha} \rho\right)(\alpha) \stackrel{(\star)}{=} k \rho(\alpha)=k(\rho \circ \Phi)(x) .
$$

The equality $(\star)$ is Euler's formula, which holds for any homogeneous function $\rho$ of degree $k$ on a vector space.

We close the section with some observations concerning the smoothness of the localizing set.

Remark 2.32. A localizing set that is associated with a constant taming map as in Example 2.26 is always smooth. Indeed, for a torus $G$ and a constant taming map $v \equiv \eta \in \mathfrak{g}$, the localizing set $Z$ is the fixed point set of the closure in $G$ of the one-parameter subgroup generated by $\eta$. Because $Z$ is the fixed point set of a compact group action, its connected components are smooth submanifolds.

On the other hand, if the taming map $v$ is associated to the norm-square of a momentum map as in Example 2.27, then $Z=\operatorname{Crit}\left(\|\Phi\|^{2}\right)$ need not be smooth. For example, consider $S^{2} \times S^{2}$ equipped with the standard area form on each factor and the diagonal circle action. Denote by $N$ and $S$ the north and south poles of $S^{2}$. By the local normal form theorem, we can identify a neighborhood of the point $(N, S)$ in $S^{2} \times S^{2}$ with a neighborhood of the origin in $\mathbb{C}^{2}$, where the circle group acts on $\mathbb{C}^{2}$ with the weights $1,-1$ and with the momentum map $\Phi(z, w)=-\frac{1}{2}\|z\|^{2}+\frac{1}{2}\|w\|^{2}$. The critical set of $\|\Phi\|^{2}$ on $\mathbb{C}^{2}$ is the zero level set $\{(z, w) \mid\|z\|=\|w\|\}$, which is a cone over $S^{1} \times S^{1}$. Thus, $Z$ is not smooth at $(N, S)$.

\section{Polarized completions}

In this section, we use the taming maps introduced in Section 2 in order to introduce and develop the notion of polarized completions. This notion is the technical tool that allows us to both state and prove our localization formulae in Sections 4 and 5.

We begin with some motivation. Recall from Section 2 that the property of being $v$-polarized is crucial for our theory due to its link to the properness of $\Phi$. On the other hand, in the course of our analysis below, we will 
encounter Hamiltonian $G$-manifolds $(N, \omega, \Phi)$ and taming maps $v: N \rightarrow \mathfrak{g}$ such that the restriction to $\Phi$ to a closed subset $Y$ of $N$ is $v$-polarized, but $\Phi$ is not $v$-polarized on all of $N$. For example, this may happen if $Y$ is a closed subset (e.g., a localizing set) of a $v$-polarized Hamiltonian $G$-manifold and $N$ is a small open neighborhood of $Y$. In such situations we wish to find a closed 2-form $\widetilde{\omega}$ and momentum map $\widetilde{\Phi}$ on $N$ that agree with $\omega$ and $\Phi$ on $Y$ and such that $\widetilde{\Phi}$ is $v$-polarized on $N$. Since we do not require $Y$ to be a manifold (see Remark 2.32), we must first make precise what we mean by the condition that differential forms "agree on $Y$ ". We take the diffeological approach:

Definition 3.1. Let $N$ be a manifold and $Y$ a subset of $N$. Let $\alpha_{0}$ and $\alpha_{1}$ be differential forms on $N$, possibly of mixed degree and with coefficients in a vector space other than $\mathbb{R}$ (such as in the case of equivariant differential forms, as recalled in Section 4). We say that $\alpha_{0}$ and $\alpha_{1}$ agree on $\mathbf{Y}$ if for any positive integer $k$, any open subset $U$ of $\mathbb{R}^{k}$, and any smooth map $p: U \rightarrow N$ whose image is contained in $Y$, the pullbacks of $\alpha_{0}$ and $\alpha_{1}$ to $U$ coincide, i.e., $p^{*} \alpha_{0}=p^{*} \alpha_{1}$ as differential forms on $U$.

Remark 3.2. If $\alpha_{0}$ and $\alpha_{1}$ agree on a neighborhood of $Y$ in $N$, then they agree on $Y$. If $Y$ is a submanifold of $N$, then $\alpha_{0}$ and $\alpha_{1}$ agree on $Y$ exactly if their pullbacks to $Y$ coincide. In practice, these are the only two cases that we need.

We can now define $v$-polarized completions:

Definition 3.3. Let $(N, \omega, \Phi)$ be a Hamiltonian $G$-manifold, possibly with boundary, and let $v: N \rightarrow \mathfrak{g}$ be a taming map. Let $Y$ be a $G$-invariant closed subset of $N$. Suppose that the restriction of $\Phi$ to $Y$ is $v$-polarized. A $v$-polarized completion of $(\mathbf{N}, \boldsymbol{\omega}, \boldsymbol{\Phi})$ relative to $\mathbf{Y}$ is a Hamiltonian $G$ manifold $(N, \widetilde{\omega}, \widetilde{\Phi})$ with the same underlying manifold $N$, such that $\widetilde{\Phi}$ is $v$-polarized and such that $\widetilde{\omega}+\widetilde{\Phi}$ agrees with $\omega+\Phi$ on $Y$.

The following proposition is the main result of this section. By Definition 3.1 and Remark 3.2, the proposition gives a $v$-polarized completion of $(N, \omega, \Phi)$ relative to $Y$.

Proposition 3.4. Let $G$ be a compact Lie group, let $N$ be a $G$-manifold, possibly with boundary, and let $v: N \rightarrow \mathfrak{g}$ be a taming map. Let $Z=\left\{v^{\sharp}=0\right\}$ be the corresponding localizing set. Let $Y$ be a closed $G$-invariant subset of $N$ that contains $Z$. Let $\omega$ be a G-invariant closed 2 -form on $N$ and $\Phi$ a 
corresponding momentum map. Suppose that the restriction of $\Phi$ to $Y$ is $v$-polarized. Then there exists an invariant closed 2 -form $\widetilde{\omega}$ on $N$ and corresponding momentum map $\widetilde{\Phi}$ that coincide with $\omega$ and $\Phi$ on a G-invariant neighborhood of $Y$ and such that $\widetilde{\Phi}$ is v-polarized on $N$.

The remainder of this section is devoted to the proof of Proposition 3.4. We begin with four elementary lemmas about real valued functions. The first lemma asserts that a convex combination of functions that are proper and bounded from below is still proper and bounded from below:

Lemma 3.5. Let $N$ be a topological space. Let $f, g: N \rightarrow \mathbb{R}$ be continuous functions that are proper and bounded from below. Let $\rho_{1}, \rho_{2}: N \rightarrow \mathbb{R}$ be continuous functions that satisfy $\rho_{1} \geq 0, \rho_{2} \geq 0$, and $\rho_{1}+\rho_{2} \equiv 1$. Then the function

$$
\rho_{1} f+\rho_{2} g: N \rightarrow \mathbb{R}
$$

is proper and bounded from below.

Proof. Let $\psi$ denote the function $\rho_{1} f+\rho_{2} g$. The function $\psi$ is bounded from below by $\min (\inf f$, inf $g)$. For any $x \in N$ and $b \in \mathbb{R}$, if $\psi(x) \leq b$ then either $f(x) \leq b$ or $g(x) \leq b$. So, for any $a<b$,

$$
\psi^{-1}([a, b]) \subset f^{-1}([\inf f, b]) \cup g^{-1}([\inf g, b]) .
$$

The union on the right-hand side is compact because $f$ and $g$ are proper. Being a closed subset of a compact set, $\psi^{-1}([a, b])$ is compact. Because the interval $[a, b]$ was arbitrary, this shows that $\psi$ is proper.

The second lemma states that if a function is proper and bounded from below then its average with respect to a compact group action is also proper and bounded below:

Lemma 3.6. Let $G$ be a compact Lie group and $N$ a topological space with a $G$-action. Let $f: N \rightarrow \mathbb{R}$ be a continuous function that is proper and bounded from below. Then its $G$-average $\bar{f}: N \rightarrow \mathbb{R}$, defined by

$$
\bar{f}(x):=\int_{g \in G} f(g \cdot x) d g
$$

where $d g$ denotes the Haar probability measure on $G$, is also proper and bounded from below. 
Proof. Every lower bound for $f$ is also a lower bound for $\bar{f}$. For any $x \in N$ and $b \in \mathbb{R}$, if $\bar{f}(x) \leq b$, then there exists $g \in G$ such that $f(g \cdot x) \leq b$, and so $x \in g^{-1} \cdot\left(f^{-1}([\inf f, b])\right)$. Hence, for any $a<b$,

$$
\bar{f}^{-1}([a, b]) \subset \bigcup_{g \in G} g^{-1} \cdot f^{-1}([\inf f, b]) .
$$

The right-hand side of (3.7) is the image of the compact set $G \times f^{-1}([\inf f, b])$ under the continuous map $G \times N \rightarrow N,(g, \alpha) \mapsto g^{-1} \cdot \alpha$. Being a closed subset of a compact set, the left-hand side is also compact, as desired.

Next, we show that it is possible to expand slightly the set on which a function is proper and bounded from below:

Lemma 3.8. Let $N$ be a locally compact topological space and $f: N \rightarrow \mathbb{R}$ a continuous function. Let $Y \subset N$ be a closed subset, and suppose that the restriction $\left.f\right|_{Y}: Y \rightarrow \mathbb{R}$ is proper and bounded from below. Then there exists an open neighborhood $U_{Y}$ of $Y$ in $N$ such that the restriction of $f$ to the closure of $U_{Y}$ in $N$,

$$
\left.f\right|_{\bar{U}_{Y}}: \bar{U}_{Y} \rightarrow \mathbb{R},
$$

is also proper and bounded from below.

Proof. By local compactness, for each point $y$ in $Y$ we may choose an open neighborhood $U_{y}$ in $N$ whose closure in $N$ is compact and such that $\mid f(u)-$ $f(y) \mid<1$ for all $u \in U_{y}$.

Let $\ell$ be any integer. Because $f$ is proper on $Y$, the intersection $f^{-1}([\ell, \ell+$ 1]) $\cap Y$ is compact, so it is covered by finitely many of the sets $U_{y}$ for $y \in f^{-1}([\ell, \ell+1]) \cap Y$. Let $U_{\ell}$ denote the union of the elements of such a finite cover. Then $f\left(U_{\ell}\right) \subset[\ell-1, \ell+2]$, and the closure $\overline{U_{\ell}}$ is compact, by construction of the sets $U_{y}$.

Consider $U_{Y}:=\bigcup\left\{U_{\ell} \mid \ell \in \mathbb{Z}\right\}$. Because the $U_{\ell}$ form a locally finite collection of subsets of $N$, the closure of their union is the union of their closures: $\overline{U_{Y}}=\bigcup\left\{\overline{U_{\ell}} \mid \ell \in \mathbb{Z}\right\}$.

Let $[a, b] \subset \mathbb{R}$ be any interval in $\mathbb{R}$. We wish to show that $f^{-1}([a, b]) \cap \overline{U_{Y}}$ is compact. First, observe that $f^{-1}([a, b]) \cap \overline{U_{\ell}}$ is non-empty only if $[a, b]$ meets $[\ell-1, \ell+2]$, which occurs for only finitely many integers $\ell$. Hence, the intersection $f^{-1}([a, b]) \cap \overline{U_{Y}}$ is contained in a finite union of the sets $\overline{U_{\ell}}$. Being a closed subset of a finite union of compact sets, this intersection is compact. This shows that $f$ is proper on $\overline{U_{Y}}$, as desired. 
Finally, let $B$ be a lower bound for $f$ on $Y$; then $B-1$ is a lower bound for $f$ on $\overline{U_{Y}}$, by construction of the sets $U_{y}$. Hence $f$ is also bounded below on $\overline{U_{Y}}$. This completes the proof.

The previous lemmas are quite general and apply to topological spaces that are not necessarily manifolds. In preparation for proving Proposition 3.4, we now return to the setting of manifolds and prove a variant of Proposition 3.4 that applies to real-valued functions:

Lemma 3.9. Let $N$ be a G-manifold, possibly with boundary. Let $Y$ be a $G$-invariant closed subset of $N$, and let $f: N \rightarrow \mathbb{R}$ be a smooth $G$-invariant function such that the restriction $\left.f\right|_{Y}: Y \rightarrow \mathbb{R}$ is proper and bounded from below. Then there exists a smooth real valued $G$-invariant function on $N$ that is proper and bounded from below and that coincides with $f$ on some $G$-invariant open neighborhood of $Y$ in $N$.

Proof. By Lemma 3.8, there exists an open neighborhood $U_{Y}$ of $Y$ such that the restriction of $f$ to $\overline{U_{Y}}$ is proper and bounded from below. Let $g: N \rightarrow \mathbb{R}$ be an arbitrary smooth function that is proper and bounded from below (see $\left[19\right.$, p. 53]). Let $\rho_{1}$ and $\rho_{2}$ be a smooth partition of unity subordinate to the open covering $\left\{U_{Y}, N \backslash Y\right\}$ of $N$ and let

$$
\psi^{\prime}:=\rho_{1} f+\rho_{2} g: N \rightarrow \mathbb{R}
$$

The manifold $N$ decomposes as the union of the two closed subsets

$$
N=\bar{U}_{Y} \cup\left(N \backslash U_{Y}\right)
$$

On $N \backslash U_{Y}$, the function $\psi^{\prime}$ coincides with $g$. Because $g$ is proper and bounded from below and $N \backslash U_{Y}$ is closed in $N$, it follows that $\left.\psi^{\prime}\right|_{N \backslash U_{Y}}$ is proper and bounded from below. On the set $\bar{U}_{Y}$, the function $\psi^{\prime}$ is a convex combination of the functions $f$ and $g$, both of which are proper and bounded from below on $\bar{U}_{Y}$. By Lemma 3.5, it follows that $\left.\psi^{\prime}\right|_{\bar{U}_{Y}}$ is also proper and bounded from below and hence that $\psi^{\prime}$ is proper and bounded from below on all of $N$.

Finally, since supp $\rho_{2} \subset N \backslash Y$ and $N$ is Hausdorff, there exists some open neighborhood $U^{\prime}$ of $Y$ such that supp $\rho_{2} \cap U^{\prime}=\emptyset$, hence in particular $\psi^{\prime} \equiv f$ on $U^{\prime}$.

We now define $\psi$ to be the $G$-average of $\psi^{\prime}$. We claim that the function $\psi$ satisfies the conditions of the lemma. By Lemma 3.6, $\psi$ is still proper 
and bounded from below on $N$. Moreover, since $\psi^{\prime} \equiv f$ on $U^{\prime}$ and $f$ is $G$-invariant, $\psi$ coincides with $f$ on the intersection

$$
\bigcap_{g \in G} g \cdot U^{\prime} .
$$

This intersection is clearly $G$-invariant. It is a neighborhood of $Y$ because its complement, being the image of the closed set $G \times\left(N \backslash U^{\prime}\right)$ under the proper map $(g, x) \mapsto g \cdot x$ from $G \times N$ to $N$, is closed. This concludes the proof.

We are ready to prove the main result of this section:

Proof of Proposition 3.4. We first observe that the function $\Phi^{v}:=\langle\Phi, v\rangle$ : $N \rightarrow \mathbb{R}$ is $G$-invariant, since both $\Phi$ and $v$ are $G$-equivariant by assumption. Applying Lemma 3.9 to $\Phi^{v}$ and $Y$, we conclude that there exists a $G$-invariant function $\psi: N \rightarrow \mathbb{R}$ that is proper, bounded from below, and coincides with $\Phi^{v}$ on a $G$-invariant neighborhood $U$ of $Y$.

Let $Z=\left\{v^{\sharp}=0\right\}$ be the localizing set and let $g_{N}$ denote a choice of a $G$-invariant Riemannian metric on $N$. Consider the $G$-invariant 1-form $\Theta$ on $N \backslash Z$ defined by

$$
\Theta(\cdot)=\frac{g_{N}\left(\cdot, v^{\sharp}\right)}{g_{N}\left(v^{\sharp}, v^{\sharp}\right)} .
$$

Note that $\Theta$ has the property that $\Theta\left(v^{\sharp}\right) \equiv 1$ on $N \backslash Z$. Such a 1 -form is sometimes called a Bott projector (see, e.g., [10]), following Bott [5]. Since $\psi-\Phi^{v}$ is identically zero on an open set that contains $Y$ and hence $Z$, the product $\left(\psi-\Phi^{v}\right) \Theta$ defines a $G$-invariant 1-form on all of $N$ that vanishes on $Z$.

We will now explicitly construct a 2 -form $\widetilde{\omega}$ and momentum map $\widetilde{\Phi}$ that satisfy the conditions of the proposition. Let

$$
\widetilde{\omega}:=\omega-d\left(\left(\psi-\Phi^{v}\right) \Theta\right)
$$

and

$$
\widetilde{\Phi}^{X}:=\Phi^{X}+\left(\psi-\Phi^{v}\right) \Theta\left(X^{\sharp}\right) \quad \text { for } X \in \mathfrak{g} .
$$

On the neighborhood $U$ of $Y$ on which $\psi$ coincides with $\Phi^{v}$, we have that $\widetilde{\omega}$ and $\widetilde{\Phi}$ coincide with $\omega$ and $\Phi$, respectively. Since $\omega$ is closed, the form $\widetilde{\omega}$ is also closed. Moreover, since both $\omega$ and $\left(\psi-\Phi^{v}\right) \Theta$ are $G$-invariant, 
it follows that $\widetilde{\omega}$ is $G$-invariant. The $G$-equivariance of $\widetilde{\Phi}$ follows from the $G$-equivariance of $\Phi$, the $G$-invariance of $\left(\psi-\Phi^{v}\right) \Theta$, and the $G$-equivariance of the map $X \mapsto X^{\sharp}$. Hamilton's equation (2.17) is satisfied by $\widetilde{\omega}$ and $\widetilde{\Phi}$, as can be checked as follows:

$$
\begin{aligned}
d\left(\widetilde{\Phi}^{X}\right) & =d\left(\Phi^{X}+\left(\psi-\Phi^{v}\right) \Theta\left(X^{\sharp}\right)\right) \\
& =d\left(\Phi^{X}\right)+d\left(\imath\left(X^{\sharp}\right)\left(\left(\psi-\Phi^{v}\right) \Theta\right)\right) \\
& =\imath\left(X^{\sharp}\right) \omega-\imath\left(X^{\sharp}\right) d\left(\left(\psi-\Phi^{v}\right) \Theta\right) \\
& =\imath\left(X^{\sharp}\right) \widetilde{\omega},
\end{aligned}
$$

where the second to last equality uses the $G$-invariance of the 1 -form $(\psi-$ $\left.\Phi^{v}\right) \Theta$ and the Cartan formula for the Lie derivative, $L_{X^{\sharp}}=d \iota\left(X^{\sharp}\right)+\iota\left(X^{\sharp}\right) d$. On the set $N \backslash Z$, by definition of the Bott projector,

$$
\begin{aligned}
\widetilde{\Phi}^{v} & =\Phi^{v}+\left(\psi-\Phi^{v}\right) \Theta\left(v^{\sharp}\right) \\
& =\Phi^{v}+\left(\psi-\Phi^{v}\right) \\
& =\psi .
\end{aligned}
$$

On the set $U$, we have $\psi-\Phi^{v} \equiv 0$, so $\widetilde{\Phi}^{v}=\Phi^{v}=\psi$. Since $N=U \cup(N \backslash Z)$, we conclude that $\widetilde{\Phi}^{v} \equiv \psi$ on all of $N$. Since $\psi$ is proper and bounded below, this implies $\widetilde{\Phi}$ is $v$-polarized on $N$. The result follows.

\section{Localization formulae for the DH distribution}

The main result of this section, Theorem 4.24, is a localization formula that expresses the DH distribution of a Hamiltonian $G$-manifold in terms of data near a localizing set arising from an arbitrary taming map. We begin by recalling the definition of the $\mathrm{DH}$ distribution:

Definition 4.1. Let $(M, \omega, \Phi)$ be a $2 n$-dimensional proper Hamiltonian $G$-manifold (see Definition 2.19). The DH distribution, $\mathrm{DH}_{(\mathrm{M}, \omega, \Phi)}$ : $\mathrm{C}_{\mathrm{c}}^{\infty}\left(\mathfrak{g}^{*}\right) \rightarrow \mathbb{R}$, is the distribution on $\mathfrak{g}^{*}$ that associates to any compactly supported test function $\varphi \in C_{\mathrm{C}}^{\infty}\left(\mathfrak{g}^{*}\right)$ on $\mathfrak{g}^{*}$ the real number

$$
\operatorname{DH}_{(M, \omega, \Phi)}(\varphi):=\int_{M}(\varphi \circ \Phi) \frac{\omega^{n}}{n !} .
$$

(The right-hand side is well defined because $\varphi$ is compactly supported and $\Phi$ is proper.) 
In the next lemma we observe that the $\mathrm{DH}$ distribution associated to the boundary of an odd-dimensional Hamiltonian $G$-manifold must be identically zero. The lemma is an easy consequence of Stokes' theorem. The argument is the same as that given in [16, Section 2.3]; we briefly recount the proof here for the reader's convenience (compare also to the arguments in Section 5) and since this idea is central to our cobordism arguments.

Lemma 4.2. Let $(W, \omega, \Phi)$ be a $(2 n+1)$-dimensional proper Hamiltonian $G$-manifold with boundary $\partial W$. Let $\omega_{\partial W}$ and $\Phi_{\partial W}$ denote the pullbacks of $\omega$ and $\Phi$ to the boundary. Then the DH distribution associated to the Hamiltonian $G$-manifold $\left(\partial W, \omega_{\partial W}, \Phi_{\partial W}\right)$ is identically zero.

Proof. Let $\varphi \in C_{c}^{\infty}\left(\mathfrak{g}^{*}\right)$. We have

$\mathrm{DH}_{\left(\partial \mathrm{W}, \omega_{\partial \mathrm{W}}, \Phi_{\partial \mathrm{W}}\right)}(\varphi)=\int_{\partial W} \varphi \circ \Phi \frac{\omega^{n}}{n !} \quad$ by definition of the $\mathrm{DH}$ distribution

$$
\begin{aligned}
& =\int_{W} d\left(\varphi \circ \Phi \frac{\omega^{n}}{n !}\right) \quad \text { by Stokes' theorem } \\
& =\int_{W} d(\varphi \circ \Phi) \wedge \frac{\omega^{n}}{n !} \quad \text { since } \omega \text { is closed } \\
& =\int_{W} d \varphi \circ\left(d \Phi \wedge \frac{\omega^{n}}{n !}\right) \quad \text { by the chain rule, }
\end{aligned}
$$

where $d \Phi$ is understood to be a $\mathfrak{g}^{*}$-valued 1 -form on $W$. For every $X \in \mathfrak{g}$, we have $\langle d \Phi, X\rangle=d \Phi^{X}=\imath\left(X^{\sharp}\right) \omega$, so

$$
\left\langle d \Phi \wedge \frac{\omega^{n}}{n !}, X\right\rangle=\imath\left(X^{\sharp}\right) \omega \wedge \frac{\omega^{n}}{n !}=\imath\left(X^{\sharp}\right) \frac{\omega^{n+1}}{(n+1) !}=0,
$$

because $\omega^{n+1}$ is a form of degree $2 n+2$ on the $(2 n+1)$-dimensional manifold $W$. Thus, the $\mathfrak{g}^{*}$-valued $(2 n+1)$-form $d \Phi \wedge \frac{\omega^{n}}{n !}$ vanishes when paired with any $X \in \mathfrak{g}$. We conclude that (4.3) vanishes for any test function $\varphi$, as required.

In view of Lemma 4.2 above, we recall the following definition from [16, Chap. 2, Def. 2.20]:

Definition 4.5. A proper Hamiltonian cobordism between Hamiltonian $G$-manifolds $\left(M_{0}, \omega_{0}, \Phi_{0}\right)$ and $\left(M_{1}, \omega_{1}, \Phi_{1}\right)$ is a proper Hamiltonian 
$G$-manifold with boundary $(W, \widetilde{\omega}, \widetilde{\Phi})$ and an orientation preserving diffeomorphism

$$
i: \quad-M_{0} \sqcup M_{1} \rightarrow \partial W
$$

(where the negative sign denotes opposite orientation) such that

$$
i^{*}(\widetilde{\omega}+\widetilde{\Phi})=\left(\omega_{0}+\Phi_{0}\right) \sqcup\left(\omega_{1}+\Phi_{1}\right) .
$$

The next proposition is essential for what follows. The idea of our main theorem (Theorem 4.24) is to construct a proper Hamiltonian cobordism between a given Hamiltonian $G$-manifold $(M, \omega, \Phi)$ and another Hamiltonian $G$-manifold that is described only in terms of local data near $Z$.

Proposition 4.6. Let $\left(M_{0}, \omega_{0}, \Phi_{0}\right)$ and $\left(M_{1}, \omega_{1}, \Phi_{1}\right)$ be two evendimensional Hamiltonian $G$-manifolds. Suppose that there exists a proper Hamiltonian cobordism between them. Then $\left(M_{0}, \omega_{0}, \Phi_{0}\right)$ and $\left(M_{1}, \omega_{1}, \Phi_{1}\right)$ are proper Hamiltonian $G$-manifolds and

$$
\mathrm{DH}_{\left(\mathrm{M}_{0}, \omega_{0}, \Phi_{0}\right)}=\mathrm{DH}_{\left(\mathrm{M}_{1}, \omega_{1}, \Phi_{1}\right)} .
$$

Proof. The first assertion is immediate from the definition of a proper Hamiltonian cobordism, since each boundary component is a closed subset of the cobording manifold. The second assertion follows from Lemma 4.2.

Remark 4.7. We can compose cobordisms (see [9]) after choosing "trivializations" of tubular neighborhoods of the boundary components. Thus the existence of a proper Hamiltonian cobordism is an equivalence relation on Hamiltonian $G$-manifolds with proper momentum maps.

In the previous section, we define the notion of a polarized completion of a Hamiltonian $G$-manifold with respect to a closed subset. This notion is used in the statement of the main theorem of this section, since the righthand side of the formula (4.25) is the $\mathrm{DH}$ distribution $\mathrm{DH}_{\left(\mathrm{U}_{\mathrm{Z}}, \omega_{\mathrm{Z}}, \Phi_{\mathrm{Z}}\right)}$ of a polarized completion $\left(U_{Z}, \omega_{Z}, \Phi_{Z}\right)$ of a neighborhood $U_{Z}$ of the localizing set $Z$ (see Def. 4.21). Moreover, an implicit assertion in the statement of Theorem 4.24 is that this $\mathrm{DH}$ distribution $\mathrm{DH}_{\left(\mathrm{U}_{Z}, \omega_{Z}, \Phi_{Z}\right)}$ is in fact independent of the choice of polarized completion in a sense that we make precise below. The justification of this last assertion will involve two main ingredients. First, we need to place restrictions on the neighborhood $U_{Z}$. Second, we use the invariance of equivariant cohomology under equivariant homotopy and the Cartan model for equivariant cohomology. Definition 4.8 explains the first of these two ingredients: 
Definition 4.8. Let $N$ be a $G$-manifold and $Z$ a $G$-invariant closed subset. A smooth equivariant weak deformation retraction of $N$ to $Z$ is an equivariant smooth homotopy $p_{t}: N \rightarrow N$, for $0 \leq t \leq 1$, such that

- $p_{0}$ is equal to the identity map on $N$,

- $p_{1}$ maps $N$ to $Z$,

- for all $t$, the map $p_{t}$ sends $Z$ to $Z$.

(A smooth homotopy is a homotopy such that the map $[0,1] \times N \rightarrow N$ defined by $(t, x) \mapsto p_{t}(x)$ is smooth. The homotopy is equivariant if this $\operatorname{map}[0,1] \times N \rightarrow N$ is equivariant, where $G$ acts on the product $[0,1] \times N$ by the given action on $N$ and trivially on the first factor.)

Remark 4.9. When $t=1$, the map $p_{1}$ may be viewed as a map from $N$ to $Z$. This map $p_{1}$ is, in particular, a homotopy inverse to the inclusion map $i: Z \rightarrow N$ since, by assumption, the composition $i \circ p_{1}: N \rightarrow N$ is homotopic through the maps $p_{t}: N \rightarrow N$ to the identity map on $N$, and the composition $p_{1} \circ i: Z \rightarrow Z$ is homotopic through the maps $p_{t} \circ i: Z \rightarrow Z$ to the identity map on $Z$. In particular, this implies that the restriction map $H_{G}^{*}(N) \rightarrow H_{G}^{*}(Z)$ is an isomorphism. Here $H_{G}^{*}(Z)$ is understood to be the singular (not de Rham) Borel-equivariant cohomology of $Z$, since we do not assume that $Z$ is a manifold.

The next two remarks compare Definition 4.8 to related notions that appear in the literature.

Remark 4.10. In Definition 4.8, the map $p_{1}: N \rightarrow Z$ is not required to be a retraction, i.e., its restriction to $Z$ is not required to be the identity map on $Z$. Such a requirement is too stringent for our purposes. The difference between the two notions may be seen in the following example. There is no smooth retraction from $\mathbb{R}^{2}$ to the union of the two coordinate axes, since if $p$ is a smooth map that fixes the two coordinate axes, then its differential at the origin must be the identity, so $p$ must be a diffeomorphism near the origin and it cannot be a retraction to the union of the axes. However, it is possible to construct a smooth weak deformation retraction in the sense of Definition 4.8 from $\mathbb{R}^{2}$ to the union of the coordinate axes.

Remark 4.11. Definition 4.8 is the smooth equivariant analogue of a "deformation retraction in the weak sense", in the sense of Hatcher in [23, Chapter 0, Exercise 4]. We also note that the phrase "weak deformation 
retraction" sometimes refers to a continuous map $p: N \rightarrow Z$ that is a homotopy inverse to the inclusion map $Z \hookrightarrow N$, but in which the intermediate maps $N \rightarrow N$ in the homotopy of $i \circ p$ to the identity are not required to carry $Z$ to itself.

We will work with situations in which $Z$ is an invariant closed subset of a $G$-manifold $M$ and $N$ is a $G$-invariant neighborhood of $Z$ in $M$ that admits a smooth equivariant weak deformation retraction to $Z$.

We now briefly recall the Cartan model for Borel-equivariant cohomology (with $\mathbb{R}$ coefficients). Let $M$ be a $G$-manifold. Then an equivariant differential form on $M$ is a $G$-equivariant polynomial function from the Lie algebra $\mathfrak{g}$ to the space $\Omega^{*}(M)$ of differential forms on $M$. Identifying polynomial $\mathbb{R}$-valued functions on $\mathfrak{g}$ with the symmetric algebra $S\left(\mathfrak{g}^{*}\right)$, we may think of an equivariant differential form $\alpha$ as an element of $\Omega^{*}(M) \otimes S\left(\mathfrak{g}^{*}\right)$. The $G$-equivariance condition ensures that $\alpha$ is an element of the $G$-invariants in the tensor product, where $G$ acts diagonally on each factor in the standard fashion. Hence

$$
\Omega_{G}^{*}(M) \cong\left(\Omega^{*}(M) \otimes_{\mathbb{R}} S\left(\mathfrak{g}^{*}\right)\right)^{G} .
$$

Since both $\Omega^{*}(M)$ and $S\left(\mathfrak{g}^{*}\right)$ are graded rings, we may equip $\Omega_{G}^{*}(M)$ with the grading

$$
\Omega_{G}^{k}(M):=\bigoplus_{i+2 j=k}\left(\Omega^{i}(M) \otimes_{\mathbb{R}} S^{j}\left(\mathfrak{g}^{*}\right)\right)^{G} .
$$

The equivariant differential $d_{G}: \Omega_{G}^{*}(M) \rightarrow \Omega_{G}^{*+1}(M)$, the equivariant analogue of the ordinary exterior derivative operator on $\Omega^{*}(M)$, is defined by the formula

$$
\left(d_{G} \alpha\right)(X)=d(\alpha(X))-\iota_{X^{\sharp}}(\alpha(X))
$$

where $X \in \mathfrak{g}$ is a Lie algebra element and $X^{\sharp} \in \operatorname{Vect}(M)$ denotes its corresponding vector field on $M$. The equivariant differential satisfies $d_{G} \circ d_{G}=0$, so we may define the equivariant cohomology (with $\mathbb{R}$ coefficients) $H_{G}^{*}(M ; \mathbb{R})$ as the cohomology of the complex $\left(\Omega_{G}^{*}(M), d_{G}\right)$. This is naturally isomorphic to the Borel-equivariant cohomology of the $G$-space $M$ with $\mathbb{R}$ coefficients, as defined in terms of the Borel construction.

The next lemma, which is one of our main technical tools, states conditions under which a polarized completion is unique up to cobordism.

Lemma 4.12. Let $N$ be an even-dimensional oriented $G$-manifold and $Z$ a closed subset of $N$. Suppose that there exists a smooth equivariant weak deformation retraction from $N$ to $Z$. Let $v: N \rightarrow \mathfrak{g}$ be a bounded taming map 
with corresponding localizing set $\left\{v^{\sharp}=0\right\}$ equal to $Z$. Let $\omega_{0}$ and $\omega_{1}$ be closed $G$-invariant 2 -forms on $N$ and $\Phi_{0}$ and $\Phi_{1}$ corresponding momentum maps. Suppose that $\Phi_{0}$ and $\Phi_{1}$ are $v$-polarized and suppose that $\omega_{0}+\Phi_{0}$ and $\omega_{1}+$ $\Phi_{1}$ agree on $Z$ in the sense of Definition 3.1. Let $W=[0,1] \times N$, equipped with the $G$-action that is trivial on the first factor and is the given action on $N$ on the second factor. Let $i_{0}, i_{1}: N \rightarrow W$ be the inclusions at levels 0 and 1 respectively. Then there exists on $W$ a closed 2 -form $\widetilde{\omega}$ and proper momentum map $\widetilde{\Phi}: W \rightarrow \mathfrak{g}^{*}$ such that $i_{0}^{*}(\widetilde{\omega}+\widetilde{\Phi})=\omega_{0}+\Phi_{0}$ and $i_{1}^{*}(\widetilde{\omega}+\widetilde{\Phi})=$ $\omega_{1}+\Phi_{1}$. In particular, there exists a proper Hamiltonian cobordism between $\left(N, \omega_{0}, \Phi_{0}\right)$ and $\left(N, \omega_{1}, \Phi_{1}\right)$.

Applying Proposition 4.6, we immediately get the following important consequence of the lemma:

Corollary 4.13. Under the assumptions and notation of Lemma 4.12,

$$
\mathrm{DH}_{\left(\mathrm{N}, \omega_{0}, \Phi_{0}\right)}=\mathrm{DH}_{\left(\mathrm{N}, \omega_{1}, \Phi_{1}\right)}
$$

Proof of Lemma 4.12. Let $p_{t}: N \rightarrow N$, for $0 \leq t \leq 1$, be an equivariant smooth weak deformation retraction from $N$ to $Z$ according to Definition 4.8. Because the image of $p_{1}: N \rightarrow N$ is contained in $Z$, and because $\omega_{0}+\Phi_{0}$ and $\omega_{1}+\Phi_{1}$ agree on $Z$ according to Definition 3.1, the pullback $p_{1}^{*}\left(\left(\omega_{1}+\Phi_{1}\right)-\left(\omega_{0}+\Phi_{0}\right)\right)$ is zero on $N$ and in particular is equivariantly exact. Because $p_{1}$ is smoothly equivariantly homotopic to the identity map on $N$, and equivariantly homotopic maps induce the same pullback map on equivariant cohomology, $\left(\omega_{1}+\Phi_{1}\right)-\left(\omega_{0}+\Phi_{0}\right)$ is also equivariantly exact on $N$. Thus there exists a $G$-invariant 1 -form $\alpha$ on $N$ such that

$$
d \alpha=\omega_{1}-\omega_{0} \quad \text { and } \quad \alpha\left(X^{\sharp}\right)=\Phi_{0}^{X}-\Phi_{1}^{X} \quad \text { for every } X \in \mathfrak{g} .
$$

Let $t$ denote the first coordinate on the product $W=[0,1] \times N$, and let $\pi: W \rightarrow N$ denote the projection map to the second factor. Define the closed $G$-invariant 2-form

$$
\widetilde{\omega}:=\pi^{*} \omega_{0}+d\left(t \pi^{*} \alpha\right)
$$

on $W$. This has an associated momentum map $\widetilde{\Phi}: W \rightarrow \mathfrak{g}^{*}$ given by

$$
\widetilde{\Phi}^{X}:=\pi^{*} \Phi_{0}^{X}-t \pi^{*} \alpha\left(X^{\sharp}\right) .
$$

By (4.14), the function $\widetilde{\Phi}$ is equal to the convex combination $(1-t) \pi^{*} \Phi_{0}+$ $t \pi^{*} \Phi_{1}$. The functions $\pi^{*} \Phi_{0}$ and $\pi^{*} \Phi_{1}$ are $\pi^{*} v$-polarized, because $\Phi_{0}$ and 
$\Phi_{1}$ are $v$-polarized and $\pi$ is proper. By Lemma 3.5, the function $\widetilde{\Phi}$ is $\pi^{*} v$ polarized. Since $v$ is bounded by assumption, from Lemma 2.9 we conclude that $\widetilde{\Phi}$ is proper.

We have shown that $(W, \widetilde{\omega}, \widetilde{\Phi})$ is a Hamiltonian $G$-manifold with boundary whose momentum map $\widetilde{\Phi}$ is proper. Identifying the boundary $\partial W$ with $-N \sqcup N$ (where the minus sign denotes reversed orientation), the restriction of $\widetilde{\omega}$ to $\partial W$ is $\omega_{0} \sqcup \omega_{1}$ and the restriction of $\widetilde{\Phi}$ is $\Phi_{0} \sqcup \Phi_{1}$. This completes the proof.

In preparation for our arguments in Section 5 and because the generalization requires no substantial additional argument, we also consider Hamiltonian $G$-manifolds equipped with equivariant cohomology classes, namely, quadruples $(M, \omega, \Phi, A)$, where $(M, \omega, \Phi)$ is a Hamiltonian $G$-manifold and $A \in H_{G}^{*}(M)$ is an equivariant cohomology class on $M$. We define a proper Hamiltonian cobordism between two such quadruples, $\left(M_{0}, \omega_{0}, \Phi_{0}, A_{0}\right)$ and $\left(M_{1}, \omega_{1}, \Phi_{1}, A_{1}\right)$, to be a proper Hamiltonian $G$-manifold with boundary equipped with an equivariant cohomology class $(\widetilde{M}, \widetilde{\omega}, \widetilde{\Phi}, \widetilde{A})$, and a diffeomorphism $i:-M_{0} \sqcup M_{1} \rightarrow \partial \widetilde{M}$, such that

$$
i^{*} \widetilde{\omega}=\omega_{0} \sqcup \omega_{1}, \quad i^{*} \widetilde{\Phi}=\Phi_{0} \sqcup \Phi_{1}, \quad i^{*} \widetilde{A}=A_{0} \sqcup A_{1} .
$$

If there exists a proper Hamiltonian cobordism in the above sense between $\left(M_{0}, \omega_{0}, \Phi_{0}, A_{0}\right)$ and $\left(M_{1}, \omega_{1}, \Phi_{1}, A_{1}\right)$, then in particular there exists a proper Hamiltonian cobordism between $\left(M_{0}, \omega_{0}, \Phi_{0}\right)$ and $\left(M_{1}, \omega_{1}, \Phi_{1}\right)$ in the original sense of Definition 4.5. Thus, by slight abuse of language, henceforth we use the term "proper Hamiltonian cobordism" to refer to the relation defined above between Hamiltonian $G$-spaces, i.e., triples $(M, \omega, \Phi)$, and also between Hamiltonian $G$-spaces equipped with an equivariant cohomology class, i.e., quadruples $(M, \omega, \Phi, A)$ as above.

Remark 4.16. As in Remark 4.7, the existence of a proper Hamiltonian cobordism is an equivalence relation on quadruples $(M, \omega, \Phi, A)$. Indeed, we can compose two cobordisms as in Remark 4.7, and by a Mayer-Vietoris argument there exists an equivariant cohomology class on the composed cobordism that restricts to the given ones on the two pieces.

The next lemma shows that the DH distribution of a polarized completion on a neighborhood of $Z$ with respect to $Z$ is not only independent of the choice of polarized completion, as we saw in Corollary 4.13, but is also independent of the choice of the neighborhood. 
Lemma 4.17. Let $(M, \omega, \Phi)$ be a Hamiltonian $G$-manifold, $Z$ an invariant closed subset, and $A \in H_{G}^{*}(M)$ an equivariant cohomology class. Let $U_{Z}^{0}$ and $U_{Z}^{1}$ be invariant open neighborhoods of $Z$ in $M$, and suppose that there exist smooth equivariant weak deformation retractions from $U_{Z}^{0}$ and $U_{Z}^{1}$ to $Z$. Let $v: M \rightarrow \mathfrak{g}$ be a bounded taming map such that $\left\{v^{\sharp}=0\right\} \cap U_{Z}^{0}=\left\{v^{\sharp}=\right.$ $0\} \cap U_{Z}^{1}=Z$. For $j=0,1$, let $\omega_{j}$ be a closed $G$-invariant 2 -form on $U_{Z}^{j}$ and let $\Phi_{j}$ be a corresponding momentum map. For $j=0,1$, suppose that $\Phi_{j}$ is $\left.v\right|_{U_{Z}^{j}}$-polarized and that $\omega_{j}+\Phi_{j}$ agrees with $\omega+\Phi$ on $Z$ in the sense of Definition 3.1. Then there exists a proper Hamiltonian cobordism between $\left(U_{Z}^{0}, \omega_{0}, \Phi_{0},\left.A\right|_{U_{Z}^{0}}\right)$ and $\left(U_{Z}^{1}, \omega_{1}, \Phi_{1},\left.A\right|_{U_{Z}^{1}}\right)$.

Proof. The union $U:=U_{Z}^{0} \cup U_{Z}^{1}$ is a $G$-invariant open neighborhood of $Z$ in $M$ whose intersection with the localizing set $\left\{v^{\sharp}=0\right\}$ is equal to $Z$. Consider the product $U \times[0,1]$ as a $G$-manifold, with the $G$-action given by the action on the first factor.

Consider the $G$-invariant open subset $W$ of $U \times[0,1]$ defined by

$$
W:=U \times[0,1] \backslash\left(\left(\left(U \backslash U_{Z}^{0}\right) \times\{0\}\right) \sqcup\left(\left(U \backslash U_{Z}^{1}\right) \times\{1\}\right)\right)
$$

Let $\omega_{W}$ and $\Phi_{W}$ denote the pullbacks of $\omega$ and $\Phi$ under the map from $W$ to $U$ given by projecting to the first factor. Then $\left(W, \omega_{W}, \Phi_{W}\right)$ is a Hamiltonian $G$-manifold.

By slight abuse of notation we denote also by $v: W \rightarrow \mathfrak{g}$ the pullback of $v: U \rightarrow \mathfrak{g}$ by the projection $W \rightarrow U$ to the first factor. The localizing set of $(W, v)$ is then $Z \times[0,1]$. Moreover, the restriction of $\Phi_{W}$ to $Z \times[0,1]$ is $v$-polarized.

Now let $(W, \widetilde{\omega}, \widetilde{\Phi})$ be a $v$-polarized completion of $\left(W, \omega_{W}, \Phi_{W}\right)$ relative to $Z \times[0,1]$, which exists by Proposition 3.4. Identifying the boundary $\partial W$ of $W$ with $U_{Z}^{0} \sqcup U_{Z}^{1}$, the restriction of $\widetilde{\omega}+\widetilde{\Phi}$ to $\partial W$ has the form

$$
\left(\omega_{0}^{\prime}+\Phi_{0}^{\prime}\right) \sqcup\left(\omega_{1}^{\prime}+\Phi_{1}^{\prime}\right)
$$

where $\omega_{j}^{\prime}+\Phi_{j}^{\prime}$ is a polarized completion of $\left(U_{Z}^{j},\left.\omega\right|_{U_{Z}^{j}},\left.\Phi\right|_{U_{Z}^{j}}\right)$ with respect to $Z$. Taking $\widetilde{A} \in H_{G}^{*}(W)$ to be the pullback of $A \in H_{G}^{*}(M)$ through the map $(x, t) \mapsto x$, we obtain that $(W, \widetilde{\omega}, \widetilde{\Phi}, \widetilde{A})$ is a proper Hamiltonian cobordism between $\left(U_{Z}^{0}, \omega_{0}^{\prime}, \Phi_{0}^{\prime},\left.A\right|_{U_{Z}^{0}}\right)$ and $\left(U_{Z}^{1}, \omega_{1}^{\prime}, \Phi_{1}^{\prime},\left.A\right|_{U_{Z}^{1}}\right)$.

By Lemma 4.12, there also exist proper Hamiltonian cobordisms between $\left(U_{Z}^{0}, \omega_{0}^{\prime}, \Phi_{0}^{\prime},\left.A\right|_{U_{Z}^{0}}\right)$ and $\left(U_{Z}^{0}, \omega_{0}, \Phi_{0},\left.A\right|_{U_{Z}^{0}}\right)$ and between $\left(U_{Z}^{1}, \omega_{1}^{\prime}, \Phi_{1}^{\prime},\left.A\right|_{U_{Z}^{1}}\right)$ and $\left(U_{Z}^{1}, \omega_{1}, \Phi_{1},\left.A\right|_{U_{Z}^{1}}\right)$. Composing these cobordisms (see Remark 4.16) gives the desired result. 
Applying Proposition 4.6 yields the following consequence.

Corollary 4.18. Under the assumptions and notation of Lemma 4.17,

$$
\mathrm{DH}_{\left(\mathrm{U}_{\mathrm{Z}}^{0}, \omega_{0}, \Phi_{0}\right)}=\mathrm{DH}_{\left(\mathrm{U}_{\mathrm{Z}}^{1}, \omega_{1}, \Phi_{1}\right)} .
$$

Our next step is to find a proper Hamiltonian cobordism between two Hamiltonian $G$-manifolds whose DH distributions yield the left- and righthand sides of our localization formula (4.25) below. For this we again invoke the existence of $v$-polarized completions. The proof uses the same ideas as that of Lemma 4.17: we start with a trivial cobordism, remove irrelevant pieces of the boundary, and take a polarized completion.

Proposition 4.19. Let $(M, \omega, \Phi)$ be an even-dimensional Hamiltonian $G$-manifold without boundary. Let $v: M \rightarrow \mathfrak{g}$ be a bounded taming map, let $Z=\left\{v^{\sharp}=0\right\}$ be the corresponding localizing set, and let $U_{Z}$ be a $G$ invariant neighborhood of $Z$ in $M$. Suppose that $\Phi$ is v-polarized on $M$. Let $A \in H_{G}^{*}(M)$ be an equivariant cohomology class on $M$. Then there exist a $\left.v\right|_{U_{Z}}$-polarized completion $\left(U_{Z}, \omega_{Z}, \Phi_{Z}\right)$ of $\left(U_{Z},\left.\omega\right|_{U_{Z}},\left.\Phi\right|_{U_{Z}}\right)$ relative to $Z$ (in the sense of Definition 3.3) and a proper Hamiltonian cobordism between $(M, \omega, \Phi, A)$ and $\left(U_{Z}, \omega_{Z}, \Phi_{Z},\left.A\right|_{U_{Z}}\right)$.

Proof. Consider $M \times[0,1]$ as a $G$-manifold, with the $G$-action given by the action on the first factor. Let $\pi: M \times[0,1] \rightarrow M$ denote the projection to the first factor. By a slight abuse of notation we denote by $v: M \times[0,1] \rightarrow \mathfrak{g}$ the pullback of $v: M \rightarrow \mathfrak{g}$ by $\pi$. The localizing set of $(M \times[0,1], v)$ is then $Z \times[0,1]$. Consider the open subset $W$ of $M \times[0,1]$ defined by

$$
W:=M \times[0,1] \backslash\left(\left(M \backslash U_{Z}\right) \times\{0\}\right),
$$

and consider the closed subset $Y$ of $W$ defined by

$$
Y:=(Z \times[0,1]) \cup(M \times\{1\}) .
$$

Both $W$ and $Y$ are $G$-invariant since $U_{Z}$ and $Z$ are $G$-invariant. Let $\omega_{W}$ and $\Phi_{W}$ denote the pullbacks of $\omega$ and $\Phi$ under the projection map $\left.\pi\right|_{W}: W \rightarrow$ $M$. Then $\left(W, \omega_{W}, \Phi_{W}\right)$ is a Hamiltonian $G$-manifold. Moreover, since $\Phi$ is assumed to be $v$-polarized on $M$ and $\pi$ is proper, the map $\pi^{*} \Phi$ is $v$-polarized on $M \times[0,1]$, and since $Y$ is closed as a subset of $M \times[0,1]$, the restriction of $\Phi_{W}$ to $Y$ is also $v$-polarized. 
Now let $(W, \widetilde{\omega}, \widetilde{\Phi})$ be a choice of $v$-polarized completion of $\left(W, \omega_{W}, \Phi_{W}\right)$ relative to $Y$, which exists by Proposition 3.4. Identifying the boundary $\partial W$ of $W$ with $-U_{Z} \sqcup M$, the restriction of $\widetilde{\omega}+\widetilde{\Phi}$ to $\partial W$ becomes

$$
\left(\omega_{Z}+\Phi_{Z}\right) \sqcup(\omega+\Phi)
$$

where $\omega_{Z}+\Phi_{Z}$ denotes the restriction of $\widetilde{\omega}+\widetilde{\Phi}$ to the boundary component $U_{Z}$. Because $\widetilde{\Phi}$ is $v$-polarized by construction and this boundary component is a closed subset of $W$, the map $\Phi_{Z}$ is $v$-polarized. Moreover, by the construction of $\widetilde{\omega}$ and $\widetilde{\Phi}$ and by the definition of $Y$, we also have that $\omega_{Z}+\Phi_{Z}$ agrees with $\omega+\Phi$ on $Z$. Thus, $\left(U_{Z}, \omega_{Z}, \Phi_{Z}\right)$ is a $v$-polarized completion of $\left(U_{Z},\left.\omega\right|_{U_{Z}},\left.\Phi\right|_{U_{Z}}\right)$ relative to $Z$. Because $\widetilde{\Phi}$ is $v$-polarized and $v$ is bounded, $\widetilde{\Phi}$ is proper. Finally, note that the restriction to $\partial W$ of the equivariant cohomology class $\widetilde{A}:=\left.\pi\right|_{W} ^{*} A$ is $\left.A\right|_{U_{Z}} \sqcup A$. Thus, $(W, \widetilde{\omega}, \widetilde{\Phi}, \widetilde{A})$ is a proper Hamiltonian cobordism between $(M, \omega, \Phi, A)$ and $\left(U_{Z}, \omega_{Z}, \Phi_{Z},\left.A\right|_{U_{Z}}\right)$.

For the next theorem, we introduce the following notation.

Definition 4.21. Let $(M, \omega, \Phi)$ be a Hamiltonian $G$ manifold, let $v: M \rightarrow \mathfrak{g}$ be a bounded taming map, and let $Z_{i}$ be a connected component of the localizing set. Suppose that there exist arbitrarily small neighborhoods of $Z_{i}$ that admit smooth equivariant weak deformation retractions to $Z_{i}$. (This means that every neighborhood of $Z_{i}$ contains a neighborhood with this property.) Let $U_{i}$ be such a neighborhood, and let $\left(U_{i}, \omega_{i}, \Phi_{i}\right)$ be a $\left.v\right|_{U_{i}{ }^{-}}$ polarized completion of $\left(U_{i},\left.\omega\right|_{U_{i}},\left.\Phi\right|_{U_{i}}\right)$ relative to $Z_{i}$. In this situation we define the notation

$$
\mathrm{DH}_{\mathrm{germ}_{\mathrm{Z}_{\mathrm{i}}}(\mathrm{M}, \omega, \Phi)}^{\mathrm{v}}:=\mathrm{DH}_{\left(\mathrm{U}_{\mathrm{i}}, \omega_{\mathrm{i}}, \Phi_{\mathrm{i}}\right)}
$$

To justify the notation, we note that the distribution on the right-hand side of (4.22) is independent of the choice of $U_{i}$ and polarized completion $\left(\omega_{i}, \Phi_{i}\right)$ by Corollary 4.18. Moreover, it is determined by the restriction of $\omega$ and $\Phi$ to arbitrarily small neighborhoods of $Z_{i}$ because $U_{i}$ can be chosen to be arbitrarily small.

Remark 4.23. In the above discussion we made the technical hypothesis, that there exist arbitrarily small neighborhoods of $Z_{i}$ that admit a smooth equivariant weak deformation retraction to $Z_{i}$. This hypothesis is automatically satisfied when $Z_{i}$ is a manifold by choosing an invariant tubular neighborhood of $Z_{i}$. In many examples, such as those considered in Section 6 , the components $Z_{i}$ of the localizing set $Z$ are indeed smooth and hence 
manifolds. However, there are important situations in which the $Z_{i}$ are not necessarily smooth. Specifically, the critical set for the norm-square of the momentum map for a nondegenerate Hamiltonian $G$-manifold can be singular, as we saw in Remark 2.32. Nevertheless, we expect this critical set to always satisfy our technical hypothesis. A proof would construct such weak deformation retractions locally using local normal forms for Hamiltonian $G$-manifolds, and would then patch them in an appropriate sense using a partition of unity.

We now state and prove the main theorem.

Theorem 4.24. Let $(M, \omega, \Phi)$ be an even-dimensional Hamiltonian $G$-manifold without boundary. Let $v: M \rightarrow \mathfrak{g}$ be a bounded taming map and let $Z=\left\{v^{\sharp}=0\right\}$ be the corresponding localizing set. Suppose that $\Phi$ is $v$-polarized on $M$, hence on $Z$. Let

$$
Z=\bigsqcup_{i \in \mathcal{I}} Z_{i}
$$

be the decomposition of the localizing set $Z$ into its connected components. Suppose that, for every $i \in \mathcal{I}$, there exist arbitrarily small neighborhoods of $Z_{i}$ that admit smooth equivariant weak deformation retractions to $Z_{i}$. Then

$$
\mathrm{DH}_{(\mathrm{M}, \omega, \Phi)}=\sum_{\mathrm{i}} \mathrm{DH}_{\mathrm{germ}_{\mathrm{Z}_{\mathrm{i}}}}^{\mathrm{v}}(\mathrm{M}, \omega, \Phi) .
$$

Proof of Theorem 4.24. For each $i \in \mathcal{I}$, choose an invariant neighborhood $U_{i}$ of $Z_{i}$ that admits an equivariant smooth weak deformation retraction to $Z_{i}$. Moreover, choose these neighborhoods $U_{i}$ sufficiently small so that their closures are disjoint. Let $U_{Z}$ be the union of the neighborhoods $U_{i}$. Let $\left(U_{Z}, \omega_{Z}, \Phi_{Z}\right)$ be a $\left.v\right|_{U_{Z}}$-polarized completion of $\left(U_{Z},\left.\omega\right|_{U_{Z}},\left.\Phi\right|_{U_{Z}}\right)$ relative to $Z$, as obtained from Proposition 4.19. In particular, there exists a proper Hamiltonian cobordism between $(M, \omega, \Phi)$ and $\left(U_{Z}, \omega_{Z}, \Phi_{Z}\right)$. By Proposition 4.6, $(M, \omega, \Phi)$ and $\left(U_{Z}, \omega_{Z}, \Phi_{Z}\right)$ have the same $\mathrm{DH}$ distribution:

$$
\mathrm{DH}_{(\mathrm{M}, \omega, \Phi)}=\mathrm{DH}_{\left(\mathrm{U}_{\mathrm{Z}}, \omega_{\mathrm{Z}}, \Phi_{\mathrm{Z}}\right)} .
$$

Let $\omega_{i}$ and $\Phi_{i}$ be the restrictions of $\omega_{Z}$ and $\Phi_{Z}$ to the component $U_{i}$. Then the DH distribution of $\left(U_{Z}, \omega_{Z}, \Phi_{Z}\right)$ is the sum of the DH distributions 
of $\left(U_{i}, \omega_{i}, \Phi_{i}\right)$ :

$$
\mathrm{DH}_{\left(\mathrm{U}_{\mathrm{Z}}, \omega_{\mathrm{Z}}, \Phi_{\mathrm{Z}}\right)}=\sum_{\mathrm{i}} \mathrm{DH}_{\left(\mathrm{U}_{\mathrm{i}}, \omega_{\mathrm{i}}, \Phi_{\mathrm{i}}\right)} .
$$

Because $\left(U_{i}, \omega_{i}, \Phi_{i}\right)$ is a $\left.v\right|_{U_{i}}$-polarized completion of $\left(U_{i},\left.\omega\right|_{U_{i}},\left.\Phi\right|_{U_{i}}\right)$ relative to $Z_{i}$, by definition

$$
\mathrm{DH}_{\operatorname{germ}_{\mathrm{Z}_{\mathrm{i}}}(\mathrm{M}, \omega, \Phi)}^{\mathrm{v}}=\mathrm{DH}_{\left(\mathrm{U}_{\mathrm{i}}, \omega_{\mathrm{i}}, \Phi_{\mathrm{i}}\right)}
$$

Equation (4.25) follows from (4.26), (4.27), and (4.28).

Remark 4.29. In Definition 4.21, if $\left(U_{i}, \omega_{i}, \Phi_{i}\right)$ is a polarized completion of $\left(U_{i},\left.\omega\right|_{U_{i}},\left.\Phi\right|_{U_{i}}\right)$, and if $v^{\prime}$ is a taming map that is equivalent to $v$ in the sense of Remark 2.12, then $\left(U_{i}, \omega_{i}, \Phi_{i}\right)$ is also a polarized completion of

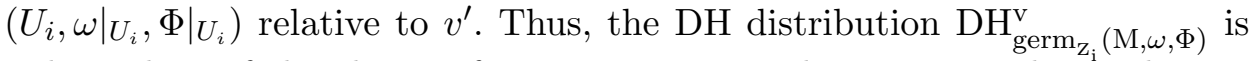
independent of the choice of taming map $v$ within an equivalence class in the sense of Remark 2.12.

\section{Localization formulae for twisted DH distributions}

The main result of this section is a localization theorem for twisted $\mathrm{DH}$ distributions (Theorem 5.20) that is analogous to Theorem 4.24.

Definitions and notation. For any real vector space $V$, there is a natural embedding $V \rightarrow \operatorname{Vect}(V)$ of $V$ into the space of smooth vector fields on $V$ by $v \mapsto \tilde{v}$, where $\tilde{v}$ denotes the constant coefficient vector field $\tilde{v}(x)=v \in$ $T_{x} V \cong V$. Furthermore, a smooth vector field $X$ on $V$ may be interpreted as an element of the space $\operatorname{LDO}(V)$ of linear differential operators on $V$ via the Lie derivative $\mathcal{L}_{X}$, so we also have an embedding $\operatorname{Vect}(V) \rightarrow \operatorname{LDO}(V)$ by the association $X \mapsto \mathcal{L}_{X}$. Because partial derivatives commute, the composition $V \rightarrow \operatorname{Vect}(V) \rightarrow \operatorname{LDO}(V)$ extends to an algebra embedding $S(V) \rightarrow$ $\operatorname{LDO}(V)$, denoted $Q \mapsto D_{Q}$, of the symmetric algebra $S(V)$ into $\operatorname{LDO}(V)$. Given an element $Q \in S(V)$, we denote by

$$
\begin{aligned}
S(V) \otimes_{\mathbb{R}} C^{\infty}(V) & \mapsto C^{\infty}(V), \\
(Q, \varphi) & \mapsto D_{Q} \varphi
\end{aligned}
$$

the pairing obtained by applying the differential operator $D_{Q}$ to the function $\varphi$. 
Consider now the case $V=\mathfrak{g}^{*}$, the dual of the Lie algebra $\mathfrak{g}$ of a compact Lie group $G$. Also, let $M$ be a $G$-manifold and $\Phi: M \rightarrow \mathfrak{g}^{*}$ a smooth map. Composing with the pullback $\Phi^{*}: C^{\infty}\left(\mathfrak{g}^{*}\right) \rightarrow C^{\infty}(M)$ yields the linear map

$$
\begin{aligned}
S\left(\mathfrak{g}^{*}\right) \otimes_{\mathbb{R}} C^{\infty}\left(\mathfrak{g}^{*}\right) & \rightarrow C^{\infty}(M) \\
(Q, \varphi) & \mapsto \Phi^{*}\left(D_{Q} \varphi\right)=D_{Q} \varphi \circ \Phi .
\end{aligned}
$$

Finally, tensoring (5.2) with the identity map on $\Omega^{*}(M)$ and composing with the pointwise multiplication map $\Omega^{*}(M) \otimes_{\mathbb{R}} C^{\infty}(M) \rightarrow \Omega^{*}(M)$, we obtain a linear map $\left(\Omega^{*}(M) \otimes_{\mathbb{R}} S\left(\mathfrak{g}^{*}\right)\right) \otimes_{\mathbb{R}} C^{\infty}\left(\mathfrak{g}^{*}\right) \rightarrow \Omega^{*}(M)$. An element $\eta \in \Omega^{*}(M) \otimes_{\mathbb{R}} S\left(\mathfrak{g}^{*}\right)$ determines via this map a linear transformation

$$
D_{\eta, \Phi}: C^{\infty}\left(\mathfrak{g}^{*}\right) \rightarrow \Omega^{*}(M),
$$

which may be expressed in explicit coordinates as follows.

We fix for the rest of this discussion a choice of basis $\left\{X_{1}, X_{2}, \ldots, X_{r}\right\}$ for $\mathfrak{g}$ and corresponding dual basis $\left\{\beta_{1}, \beta_{2}, \ldots, \beta_{r}\right\}$ of $\mathfrak{g}^{*}$. For a multi-index $\mathbf{a}=$ $\left(a_{1}, a_{2}, \ldots, a_{r}\right) \in \mathbb{Z}_{>0}^{r}$, denote by $\beta^{\mathbf{a}}$ the monomial $\beta_{1}^{a_{1}} \beta_{2}^{a_{2}} \cdots \beta_{r}^{a_{r}} \in S\left(\mathfrak{g}^{*}\right)$. An element $\eta \in \Omega^{*}(M) \otimes_{\mathbb{R}} S\left(\mathfrak{g}^{*}\right)$ may be expressed in these coordinates as

$$
\eta=\sum_{\mathbf{a}} \eta_{\mathbf{a}} \beta^{\mathbf{a}}
$$

where the coefficients $\eta_{\mathbf{a}} \in \Omega^{*}(M)$ are differential forms. Tracing through the definition of the map $D_{\eta, \Phi}$ of (5.3), an explicit computation shows that, for $\varphi \in C^{\infty}\left(\mathfrak{g}^{*}\right)$ and $\eta$ as above, the function (5.3) is given by

$$
D_{\eta, \Phi}(\varphi)=\sum_{\mathbf{a}}\left(\Phi^{*}\left(D_{\beta^{\mathbf{a}}} \varphi\right)\right) \eta_{\mathbf{a}} .
$$

We may now define the twisted DH distribution on $\mathfrak{g}^{*}$. We first place the additional assumption that $\Phi$ is proper. In this case, for any compactly supported function $\varphi$ on $\mathfrak{g}^{*}$ and any $\mathbf{a} \in \mathbb{Z}_{\geq 0}^{r}$, the functions $D_{\beta^{\mathrm{a}}} \varphi$ and $\Phi^{*}\left(D_{\beta^{\mathbf{a}}} \varphi\right)$ are also compactly supported (on $\mathfrak{g}^{*}$ and $M$ respectively), and hence $D_{\eta, \Phi}(\varphi)$ is a compactly supported differential form on $M$. In particular, its integration over $M$ is well-defined. Now let $(M, \omega, \Phi)$ be a proper Hamiltonian $G$-manifold (see Definition 2.19). Let $\eta \in\left(\Omega^{*}(M) \otimes_{\mathbb{R}} S\left(\mathfrak{g}^{*}\right)\right)^{G}$ be an equivariantly closed equivariant form on $M$. We define the twisted DH distribution with respect to $(M, \omega, \Phi)$ and $\eta$ on $\mathfrak{g}^{*}$ as follows:

$$
\operatorname{DH}_{(\mathrm{M}, \omega, \Phi)}(\eta): \varphi \mapsto \int_{\mathrm{M}} \mathrm{e}^{\omega} \wedge \mathrm{D}_{\eta, \Phi}(\varphi) .
$$


When there is no danger of ambiguity, we will occasionally abuse notation and denote by $\mathrm{DH}(\eta)$ the distribution $\mathrm{DH}_{(\mathrm{M}, \omega, \Phi)}(\eta)$.

The explicit formula (5.6) implies that $\varphi \mapsto \mathrm{DH}(\eta)(\varphi)$ is linear and continuous as a map from the space $C_{c}^{\infty}\left(\mathfrak{g}^{*}\right)$ of compactly supported functions, with its $C^{\infty}$ topology, to $\mathbb{R}$. Hence $\mathrm{DH}(\eta)$ is a distribution. Moreover, when $\eta \equiv 1$, the twisted DH distribution reduces to the classical ("untwisted") DH distribution discussed in the previous section.

Remark 5.7. When $M$ is compact, we can integrate $\exp (\omega+\mathrm{i} \Phi) \wedge \eta$ over $M$ to obtain an analytic function on $\mathfrak{g}$. The twisted $\mathrm{DH}$ distribution $\mathrm{DH}_{(\mathrm{M}, \omega, \Phi)}(\eta)$ is essentially the Fourier transform of this function. See [34, Section 3.1].

We now add some extra data to that of a Hamiltonian $G$-manifold: we call Hamiltonian $G$-manifold equipped with a closed equivariant form a quadruple $(M, \omega, \Phi, \eta)$ where $(M, \omega, \Phi)$ is a Hamiltonian $G$-manifold and $\eta \in \Omega_{G}^{*}(M)$ is an equivariant differential form on $M$ that is equivariantly closed. In analogy with the definitions in Section 4 (and analogous slight abuse of language), a proper Hamiltonian cobordism between two such quadruples $\left(M_{0}, \omega_{0}, \Phi_{0}, \eta_{0}\right)$ and $\left(M_{1}, \omega_{1}, \Phi_{1}, \eta_{1}\right)$ is a quadruple $(\widetilde{M}, \widetilde{\omega}, \widetilde{\Phi}, \widetilde{\eta})$ and a diffeomorphism $i:-M_{0} \sqcup M_{1} \rightarrow \partial \widetilde{M}$ such that

$$
i^{*} \widetilde{\omega}=\omega_{0} \sqcup \omega_{1}, \quad i^{*} \widetilde{\Phi}=\Phi_{0} \sqcup \Phi_{1}, \quad i^{*} \widetilde{\eta}=\eta_{0} \sqcup \eta_{1},
$$

and such that $(\widetilde{M}, \widetilde{\omega}, \widetilde{\Phi}, \widetilde{\eta})$ is itself a proper Hamiltonian $G$-manifold (with boundary) equipped with a closed equivariant form. Note that if such a cobordism exists, then $\Phi_{0}$ and $\Phi_{1}$ are necessarily proper.

Remark 5.9. As in Remark 4.16, after possibly modifying the equivariant differential forms on tubular neighborhoods of the boundary components, we can compose such cobordisms. Thus, being cobordant in the sense defined above is an equivalence relation on proper Hamiltonian $G$-manifolds equipped with closed equivariant forms.

We begin with the following "twisted analogue" of Lemma 4.2 and Proposition 4.6:

Lemma 5.10. Let $\left(M_{0}, \omega_{0}, \Phi_{0}, \eta_{0}\right)$ and $\left(M_{1}, \omega_{1}, \Phi_{1}, \eta_{1}\right)$ be proper Hamiltonian G-manifolds equipped with closed equivariant forms. Suppose that there exists a proper Hamiltonian cobordism between $\left(M_{0}, \omega_{0}, \Phi_{0}, \eta_{0}\right)$ and 
$\left(M_{1}, \omega_{1}, \Phi_{1}, \eta_{1}\right)$. Then

$$
\mathrm{DH}_{\left(\mathrm{M}_{0}, \omega_{0}, \Phi_{0}\right)}\left(\eta_{0}\right)=\mathrm{DH}_{\left(\mathrm{M}_{1}, \omega_{1}, \Phi_{1}\right)}\left(\eta_{1}\right)
$$

Proof. Let $(\widetilde{M}, \widetilde{\omega}, \widetilde{\Phi}, \widetilde{\eta})$, with diffeomorphism $i:-M_{0} \sqcup M_{1} \rightarrow \partial \widetilde{M}$, be a proper Hamiltonian cobordism between $\left(M_{0}, \omega_{0}, \Phi_{0}, \eta_{0}\right)$ and $\left(M_{1}, \omega_{1}, \Phi_{1}, \eta_{1}\right)$. To show that the twisted DH distributions are equal, it suffices to show that for any $\varphi \in C_{\mathrm{c}}^{\infty}\left(\mathfrak{g}^{*}\right)$ we have

$$
\operatorname{DH}_{\left(\mathrm{M}_{0}, \omega_{0}, \Phi_{0}\right)}\left(\eta_{0}\right)(\varphi)=\mathrm{DH}_{\left(\mathrm{M}_{1}, \omega_{1}, \Phi_{1}\right)}\left(\eta_{1}\right)(\varphi)
$$

We compute the difference as

$$
\begin{aligned}
\mathrm{DH}_{\left(\mathrm{M}_{1}, \omega_{1}, \Phi_{1}\right)}\left(\eta_{1}\right)(\varphi)-\mathrm{DH}_{\left(\mathrm{M}_{0}, \omega_{0}, \Phi_{0}\right)}\left(\eta_{0}\right)(\varphi) & \\
= & \int_{M_{1}} \mathrm{e}^{\omega_{1}} \wedge D_{\eta_{1}, \Phi_{1}}(\varphi)-\int_{M_{0}} \mathrm{e}^{\omega_{0}} \wedge D_{\eta_{0}, \Phi_{0}}(\varphi) \\
& =\int_{\partial \widetilde{M}} \mathrm{e}^{\widetilde{\omega}} \wedge D_{\widetilde{\eta}, \widetilde{\Phi}}(\varphi) \quad \text { by }(5.8) \\
& =\int_{\widetilde{M}} d\left(\mathrm{e}^{\widetilde{\omega}} \wedge D_{\widetilde{\eta}, \widetilde{\Phi}}(\varphi)\right) \quad \text { by Stokes' theorem. }
\end{aligned}
$$

Thus it suffices to prove that

$$
\int_{\widetilde{M}} d\left(\mathrm{e}^{\widetilde{\omega}} \wedge D_{\widetilde{\eta}, \widetilde{\Phi}}(\varphi)\right)=0 .
$$

We first write

$$
\widetilde{\eta}=\sum_{\mathbf{a}} \widetilde{\eta}_{\mathbf{a}} \beta^{\mathbf{a}}
$$

with respect to the basis $\left\{\beta_{i}\right\}$ of $\mathfrak{g}^{*}$ fixed above, where the $\widetilde{\eta}_{\mathbf{a}}$ are differential forms on $M$ (of mixed degree). Then $D_{\widetilde{\eta}, \widetilde{\Phi}}(\varphi)=\sum_{\mathbf{a}}\left(\widetilde{\Phi}^{*}\left(D_{\beta^{\mathbf{a}}} \varphi\right)\right) \widetilde{\eta}_{\mathbf{a}}$ by (5.5). Since $\widetilde{\omega}$ is closed, $d\left(e^{\widetilde{\omega}}\right)=0$, so

$$
d\left(\mathrm{e}^{\widetilde{\omega}} \wedge D_{\widetilde{\eta}, \widetilde{\Phi}}(\varphi)\right)=\mathrm{e}^{\widetilde{\omega}} \wedge d\left(D_{\widetilde{\eta}, \widetilde{\Phi}}(\varphi)\right)
$$


We compute

$$
\begin{aligned}
d\left(D_{\widetilde{\eta}, \widetilde{\Phi}}(\varphi)\right) & =d\left(\sum_{\mathbf{a}}\left(\widetilde{\Phi}^{*}\left(D_{\beta^{\mathbf{a}}} \varphi\right)\right) \widetilde{\eta}_{\mathbf{a}}\right) \\
& =\sum_{\mathbf{a}} d\left(\widetilde{\Phi}^{*}\left(D_{\beta^{\mathbf{a}}} \varphi\right)\right) \wedge \widetilde{\eta}_{\mathbf{a}}+\sum_{\mathbf{a}}\left(\widetilde{\Phi}^{*}\left(D_{\beta^{\mathbf{a}}} \varphi\right)\right) d \widetilde{\eta}_{\mathbf{a}} \\
& =\sum_{\mathbf{a}} \widetilde{\Phi}^{*}\left(d\left(D_{\beta^{\mathbf{a}}} \varphi\right) \wedge \widetilde{\eta}_{\mathbf{a}}+\sum_{\mathbf{a}}\left(\widetilde{\Phi}^{*}\left(D_{\beta^{\mathbf{a}}} \varphi\right)\right) d \widetilde{\eta}_{\mathbf{a}}\right. \\
& =\sum_{\mathbf{a}} \sum_{i} \widetilde{\Phi}^{*}\left(D_{\beta_{i}}\left(D_{\beta^{\mathbf{a}}} \varphi\right)\right) d\left\langle\widetilde{\Phi}, X_{i}\right\rangle \wedge \widetilde{\eta}_{\mathbf{a}}+\sum_{\mathbf{a}}\left(\widetilde{\Phi}^{*}\left(D_{\beta^{\mathbf{a}}} \varphi\right)\right) d \widetilde{\eta}_{\mathbf{a}} \\
& =\sum_{\mathbf{a}} \sum_{i} \widetilde{\Phi}^{*}\left(D_{\beta_{i}}\left(D_{\beta^{\mathbf{a}}} \varphi\right)\right) \iota\left(X_{i}^{\sharp}\right) \widetilde{\omega} \wedge \widetilde{\eta}_{\mathbf{a}}+\sum_{\mathbf{a}}\left(\widetilde{\Phi}^{*}\left(D_{\beta^{\mathbf{a}}} \varphi\right)\right) d \widetilde{\eta}_{\mathbf{a}} .
\end{aligned}
$$

Now recall that $\tilde{\eta}$ is equivariantly closed, so for any $X \in \mathfrak{g}$, we have

$$
d(\widetilde{\eta}(X))-\iota\left(X^{\sharp}\right) \widetilde{\eta}(X)=0 .
$$

This implies

$$
\sum_{\mathbf{a}} d \widetilde{\eta}_{\mathbf{a}} \beta^{\mathbf{a}}=\sum_{\mathbf{a}} \sum_{i} \iota\left(X_{i}^{\sharp}\right) \widetilde{\eta}_{\mathbf{a}} \beta_{i} \beta^{\mathbf{a}},
$$

where $\left\{X_{i}\right\}$ is the basis for $\mathfrak{g}$ as fixed above. Thus for any $\varphi \in C_{c}^{\infty}\left(\mathfrak{g}^{*}\right)$ we have

$$
\sum_{\mathbf{a}}\left(\widetilde{\Phi}^{*}\left(D_{\beta^{\mathbf{a}}} \varphi\right)\right) d \widetilde{\eta}_{\mathbf{a}}=\sum_{\mathbf{a}} \sum_{i}\left(\widetilde{\Phi}^{*}\left(D_{\beta_{i} \beta^{\mathbf{a}}} \varphi\right)\right) \iota\left(X_{i}^{\sharp}\right) \widetilde{\eta}_{\mathbf{a}} .
$$

Noticing that $D_{\beta_{i} \beta^{\mathrm{a}}} \varphi=D_{\beta_{i}} D_{\beta^{\mathrm{a}}} \varphi$ by definition of $D_{Q}$, we have

$$
\sum_{\mathbf{a}}\left(\widetilde{\Phi}^{*}\left(D_{\beta^{\mathbf{a}}} \varphi\right)\right) d \widetilde{\eta}_{\mathbf{a}}=\sum_{\mathbf{a}} \sum_{i}\left(\widetilde{\Phi}^{*}\left(D_{\beta_{i}} D_{\beta^{\mathbf{a}}} \varphi\right)\right) \iota\left(X_{i}^{\sharp}\right) \widetilde{\eta}_{\mathbf{a}} .
$$

Substituting into the last expression in (5.13), we obtain

$$
d\left(D_{\widetilde{\eta}, \widetilde{\Phi}}(\varphi)\right)=\sum_{i} \sum_{\mathbf{a}} \widetilde{\Phi}^{*}\left(D_{\beta_{i}} D_{\beta^{\mathbf{a}}} \varphi\right)\left(\iota\left(X_{i}^{\sharp}\right) \widetilde{\omega} \wedge \widetilde{\eta}_{\mathbf{a}}+\iota\left(X_{i}^{\sharp}\right) \widetilde{\eta}_{\mathbf{a}}\right) .
$$

Hence

$$
\mathrm{e}^{\widetilde{\omega}} \wedge d\left(D_{\widetilde{\eta}, \widetilde{\Phi}}(\varphi)\right)=\sum_{i} \sum_{\mathbf{a}} \widetilde{\Phi}^{*}\left(D_{\beta_{i}} D_{\beta^{\mathbf{a}}} \varphi\right)\left(\mathrm{e}^{\widetilde{\omega}} \wedge \iota\left(X_{i}^{\sharp}\right) \widetilde{\omega} \wedge \widetilde{\eta}_{\mathbf{a}}+\mathrm{e}^{\widetilde{\omega}} \wedge \iota\left(X_{i}^{\sharp}\right) \widetilde{\eta}_{\mathbf{a}}\right) .
$$


From the definition of the exponential

$$
\mathrm{e}^{\widetilde{\omega}}=1+\widetilde{\omega}+\frac{\widetilde{\omega}^{2}}{2 !}+\frac{\widetilde{\omega}^{3}}{3 !}+\cdots
$$

it can be seen that $\iota\left(X_{i}^{\sharp}\right) \widetilde{\omega} \wedge \mathrm{e}^{\widetilde{\omega}}=\iota\left(X_{i}^{\sharp}\right) \mathrm{e}^{\widetilde{\omega}}=\mathrm{e}^{\widetilde{\omega}} \wedge \iota\left(X_{i}^{\sharp}\right) \widetilde{\omega}$. Hence, we may further simplify (5.14) as

$$
\begin{aligned}
\mathrm{e}^{\widetilde{\omega}} \wedge d\left(D_{\widetilde{\eta}, \widetilde{\Phi}}(\varphi)\right)= & \sum_{i} \sum_{\mathbf{a}} \widetilde{\Phi}^{*}\left(D_{\beta_{i}} D_{\beta^{\mathbf{a}}} \varphi\right)\left(\left(\iota\left(X_{i}^{\sharp}\right) \mathrm{e}^{\widetilde{\omega}}\right) \wedge \widetilde{\eta}_{\mathbf{a}}\right. \\
& \left.+\mathrm{e}^{\widetilde{\omega}} \wedge\left(\iota\left(X_{i}^{\sharp}\right) \widetilde{\eta}_{\mathbf{a}}\right)\right) \\
= & \sum_{i} \sum_{\mathbf{a}} \widetilde{\Phi}^{*}\left(D_{\beta_{i}} D_{\beta^{\mathbf{a}}} \varphi\right) \iota\left(X_{i}^{\sharp}\right)\left(e^{\widetilde{\omega}} \wedge \widetilde{\eta}_{\mathbf{a}}\right) \\
= & \sum_{i} \sum_{\mathbf{a}} \iota\left(X_{i}^{\sharp}\right)\left(\widetilde{\Phi}^{*}\left(D_{\beta_{i}} D_{\beta^{\mathbf{a}}} \varphi\right)\left(e^{\widetilde{\omega}} \wedge \widetilde{\eta}_{\mathbf{a}}\right)\right) .
\end{aligned}
$$

The integral over $M$ of the right-hand side of this last equation is 0 , because the expression is the contraction by a vector field of a differential form, and hence its top degree part (which is the only part which contributes to the integral) must be 0 . Hence (5.12) vanishes, as desired.

The purpose of the next lemma is to show that the twisted DH distribution is independent of the choice of a closed equivariant form $\eta$ within an equivariant cohomology class.

Lemma 5.15. Let $(M, \omega, \Phi)$ be a proper Hamiltonian $G$-manifold. Suppose that $\eta_{0}$ and $\eta_{1}$ are closed equivariant differential forms on $M$ such that $\left[\eta_{0}\right]=\left[\eta_{1}\right] \in H_{G}^{*}(M ; \mathbb{R})$. Then there exists a proper Hamiltonian cobordism between $\left(M, \omega, \Phi, \eta_{0}\right)$ and $\left(M, \omega, \Phi, \eta_{1}\right)$.

Proof. Since $\left[\eta_{0}\right]=\left[\eta_{1}\right]$ there exists an equivariant differential form $\gamma$ on $M$ such that $\eta_{1}-\eta_{0}=d_{G} \gamma$. Equip $\widetilde{M}:=[0,1] \times M$ with the 2 -form $\widetilde{\omega}=\pi^{*} \omega$ and momentum map $\widetilde{\Phi}=\pi^{*} \Phi$, where $\pi: \widetilde{M} \rightarrow M$ is the projection map to the second factor. Consider $\widetilde{M}$ to be a $G$-manifold equipped with the given action on $M$ and the trivial action on $[0,1]$. This makes $(\widetilde{M}, \widetilde{\omega}, \widetilde{\Phi})$ a Hamiltonian $G$-space, and $\pi$ an equivariant map. Moreover, since $\Phi: M \rightarrow$ $\mathfrak{g}^{*}$ is proper, $\widetilde{\Phi}: \widetilde{M} \rightarrow \mathfrak{g}^{*}$ is also proper. 
Let $t$ denote the coordinate on the interval $[0,1]$. Define on $\widetilde{M}$ the equivariant different form

$$
\tilde{\eta}=\pi^{*} \eta_{0}+d_{G}\left(t \pi^{*} \gamma\right)
$$

Since $d_{G} \eta_{0}=0$ by assumption, $\widetilde{\eta}$ is also equivariantly closed. Define $i_{0}: \widetilde{M} \times$ $M$ and $i_{1}: \widetilde{M} \times M$ by $i_{0}(m)=(0, m)$ and $i_{1}(m)=(1, m)$. Then $i_{0}^{*} \widetilde{\omega}=i_{1}^{*} \widetilde{\omega}=$ $\omega, i_{0}^{*} \widetilde{\Phi}=i_{1}^{*} \widetilde{\Phi}=\Phi, i_{0}^{*} \widetilde{\eta}=\eta_{0}$, and $i_{1}^{*} \widetilde{\eta}=\eta_{0}+d_{G} \gamma=\eta_{1}$. This shows that $(\widetilde{M}, \widetilde{\omega}, \widetilde{\Phi}, \widetilde{\eta})$ is a proper Hamiltonian cobordism between $\left(M, \omega, \Phi, \eta_{0}\right)$ and $\left(M, \omega, \Phi, \eta_{1}\right)$, as was to be shown.

By Lemmas 5.10 and 5.15, we have just shown that the following notion is well defined:

Definition 5.16. Let $(M, \omega, \Phi)$ be a proper Hamiltonian $G$-manifold, and let $A$ be an equivariant cohomology class in $H_{G}^{*}(M)$. Let $\eta$ be an equivariant differential form on $M$ representing $A$, i.e., $A=[\eta]$. We define the twisted DH distribution with respect to $(M, \omega, \Phi)$ and the equivariant cohomology class $A$ as

$$
\mathrm{DH}_{(\mathrm{M}, \omega, \Phi)}(\mathrm{A}):=\mathrm{DH}_{(\mathrm{M}, \omega, \Phi)}(\eta)
$$

In the previous section, we introduced the notion of a proper Hamiltonian cobordism between Hamiltonian $G$-manifolds equipped with equivariant cohomology classes. Using this notion we have the following analog of Lemma 5.10:

Lemma 5.17. Let $\left(M_{0}, \omega_{0}, \Phi_{0}\right)$ and $\left(M_{1}, \omega_{1}, \Phi_{1}\right)$ be proper Hamiltonian $G$-manifolds with equivariant cohomology classes $A_{0} \in H_{G}^{*}\left(M_{0}\right)$ and $A_{1} \in$ $H_{G}^{*}\left(M_{1}\right)$. Suppose that there exists a proper Hamiltonian cobordism between the quadruples $\left(M_{0}, \omega_{0}, \Phi_{0}, A_{0}\right)$ and $\left(M_{1}, \omega_{1}, \Phi_{1}, A_{1}\right)$. Then the corresponding twisted $D H$ distributions are equal, i.e.,

$$
\operatorname{DH}_{\left(\mathrm{M}_{0}, \omega_{0}, \Phi_{0}\right)}\left(\mathrm{A}_{0}\right)=\mathrm{DH}_{\left(\mathrm{M}_{1}, \omega_{1}, \Phi_{1}\right)}\left(\mathrm{A}_{1}\right)
$$

Proof. Let $([0,1] \times M, \widetilde{\omega}, \widetilde{\Phi}, \widetilde{A})$ be a cobording quadruple, with diffeomorphism $-M_{0} \sqcup M_{1} \rightarrow \partial([0,1] \times M)$. Let $\widetilde{\eta}$ be an equivariant differential form on $[0,1] \times M$ that represents the class $\widetilde{A}$, and let $\eta_{0}$ and $\eta_{1}$ be its pullbacks to $M_{0}$ and $M_{1}$. Then $\eta_{0}$ and $\eta_{1}$ represent the classes $A_{0}$ and $A_{1}$. The result now follows from Lemma 5.10 and Definition 5.16.

In analogy with the previous section, we introduce the following notation. 
Definition 5.18. With notation as in Definition 4.21, we define

$$
\mathrm{DH}_{\operatorname{germ}_{\mathrm{Z}_{\mathrm{i}}}(\mathrm{M}, \omega, \Phi)}^{\mathrm{v}}\left(\left.\mathrm{A}\right|_{\mathrm{Z}_{\mathrm{i}}}\right):=\mathrm{DH}_{\left(\mathrm{U}_{\mathrm{i}}, \omega_{\mathrm{i}}, \Phi_{\mathrm{i}}\right)}\left(\left.\mathrm{A}\right|_{\mathrm{U}_{\mathrm{i}}}\right)
$$

The justification that this notation is well-defined follows that given in Section 4 for Definition 4.21 except that we use Lemmas 4.17 and 5.17. We may now state and prove the main theorem:

Theorem 5.20. Let $(M, \omega, \Phi)$ be an even-dimensional Hamiltonian $G$-manifold without boundary. Let $A$ be an equivariant cohomology class in $H_{G}^{*}(M)$. Let $v: M \rightarrow \mathfrak{g}$ be a bounded taming map and let $Z=\left\{v^{\sharp}=0\right\}$ be the corresponding localizing set. Suppose that $\Phi$ is v-polarized on $M$, hence on $Z$. Let

$$
Z=\bigsqcup_{i \in \mathcal{I}} Z_{i}
$$

be the decomposition of the localizing set $Z$ into its connected components. Suppose that, for every $i \in \mathcal{I}$, there exist arbitrarily small neighborhoods of $Z_{i}$ that admit smooth equivariant weak deformation retractions to $Z_{i}$. Then

$$
\mathrm{DH}_{(\mathrm{M}, \omega, \Phi)}(\mathrm{A})=\sum_{\mathrm{i}} \mathrm{DH}_{\mathrm{germ}_{\mathrm{Z}_{\mathrm{i}}}(\mathrm{M}, \omega, \Phi)}^{\mathrm{v}}\left(\left.\mathrm{A}\right|_{\mathrm{Z}_{\mathrm{i}}}\right)
$$

Proof. For each $i \in \mathcal{I}$, choose an invariant neighborhood $U_{i}$ of $Z_{i}$ that admits an equivariant smooth weak deformation retraction to $Z_{i}$. Moreover, choose these neighborhoods $U_{i}$ to be sufficiently small so that their closures are disjoint. Let $U_{Z}$ be the union of the neighborhoods $U_{i}$. Let $\left(U_{Z}, \omega_{Z}, \Phi_{Z}\right)$ be a $\left.v\right|_{U_{Z}}$-polarized completion relative to $Z$ that is obtained from Proposition 4.19. In particular, there exists a proper Hamiltonian cobordism between $\left(U_{Z}, \omega_{Z}, \Phi_{Z},\left.A\right|_{U_{Z}}\right)$ and $(M, \omega, \Phi, A)$. By Lemma 5.17, $(M, \omega, \Phi, A)$ and $\left(U_{Z}, \omega_{Z}, \Phi_{Z},\left.A\right|_{U_{Z}}\right)$ have the same twisted DH distribution:

$$
\mathrm{DH}_{(\mathrm{M}, \omega, \Phi)}(\mathrm{A})=\mathrm{DH}_{\left(\mathrm{U}_{\mathrm{Z}}, \omega_{\mathrm{Z}}, \Phi_{\mathrm{Z}}\right)}\left(\left.\mathrm{A}\right|_{\mathrm{U}_{\mathrm{Z}}}\right)
$$

Let $\omega_{i}$ and $\Phi_{i}$ be the restrictions of $\omega_{Z}$ and $\Phi_{Z}$ to the component $U_{i}$. Then the twisted DH distribution of $\left(U_{Z}, \omega_{Z}, \Phi_{Z},\left.A\right|_{U_{Z}}\right)$ is the sum of the twisted DH distributions of $\left(U_{i}, \omega_{i}, \Phi_{i},\left.A\right|_{U_{i}}\right)$ :

$$
\mathrm{DH}_{\left(\mathrm{U}_{\mathrm{Z}}, \omega_{\mathrm{Z}}, \Phi_{\mathrm{Z}}\right)}\left(\left.\mathrm{A}\right|_{\mathrm{U}_{\mathrm{Z}}}\right)=\sum_{\mathrm{i}} \mathrm{DH}_{\left(\mathrm{U}_{\mathrm{i}}, \omega_{\mathrm{i}}, \Phi_{\mathrm{i}}\right)}\left(\left.\mathrm{A}\right|_{\mathrm{U}_{\mathrm{i}}}\right)
$$


Because $\left(U_{i}, \omega_{i}, \Phi_{i}\right)$ is a $\left.v\right|_{U_{i}}$-polarized completion of $\left(U_{i},\left.\omega\right|_{U_{i}},\left.\Phi\right|_{U_{i}}\right)$ relative to $Z_{i}$,

$$
\mathrm{DH}_{\text {germ }_{\mathrm{Z}_{\mathrm{i}}}(\mathrm{M}, \omega, \Phi)}^{\mathrm{v}}(\mathrm{A})=\mathrm{DH}_{\left(\mathrm{U}_{\mathrm{i}}, \omega_{\mathrm{i}}, \Phi_{\mathrm{i}}\right)}\left(\left.\mathrm{A}\right|_{\mathrm{U}_{\mathrm{i}}}\right)
$$

Equation 5.21 follows from (5.22), (5.23), and (5.24).

Remark 5.25. As in the untwisted case (see Remark 4.29), taming maps that are equivalent in the sense of Remark 2.12 give rise to the same localization formula.

\section{The Brianchon-Gram polytope decomposition and symplectic toric manifolds}

This paper was originally motivated by a question that Shlomo Sternberg posed some years ago. We first recall the context of his question in some detail.

As was mentioned in the introduction, it is known that the AtiyahBott-Berline-Verge localization theorem in equivariant cohomology [2-4], when applied to the exponent of the equivariant symplectic form of a compact symplectic toric manifold, yields the measure-theoretic version of the Lawrence-Varchenko polytope decomposition [26, 32], when applied to the corresponding momentum polytope. As an example, figure 1 illustrates a Lawrence-Varchenko polytope decomposition that corresponds to localization on a $\mathbb{C P}^{2}$.

Motivated by this correspondence, Shlomo Sternberg pointed at a different (and classical) polytope decomposition that goes back to Brianchon and Gram [8, 17] (see also [31]) and asked the following question.

Question 6.1. (Shlomo Sternberg) Is there a localization formula on manifolds that corresponds to the Brianchon-Gram polytope decomposition in the same way that the Atiyah-Bott-Berline-Vergne localization formula corresponds to the Lawrence-Varchenko polytope decomposition?

As an example, figure 2 illustrates the Brianchon-Gram decomposition of the same polytope as in figure 1.

These decompositions can be described as follows. Let $\Delta$ be an $n$-dimensional simple polytope in $\mathbb{R}^{n}$ (see Appendix 7.3 for definitions). The 

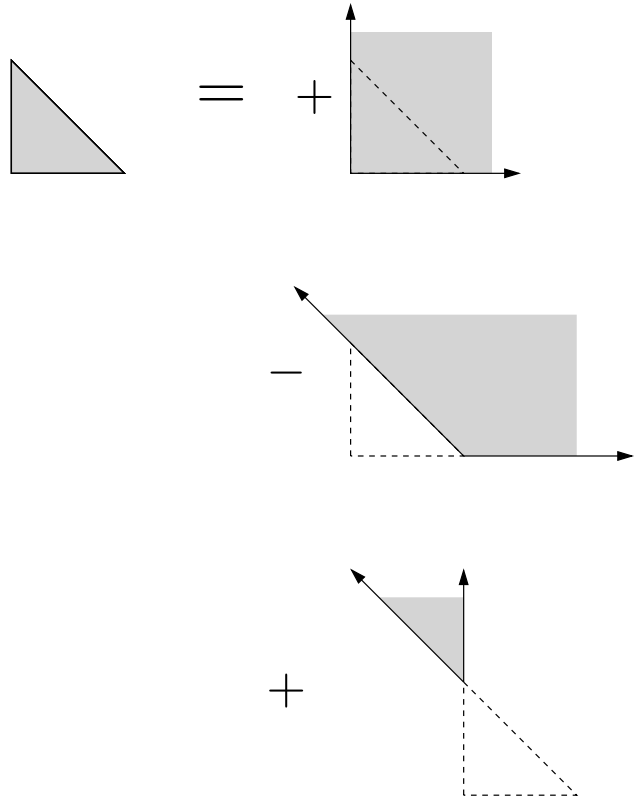

Figure 1: A Lawrence-Varchenko decomposition of a triangle. The summands on the right-hand side correspond to the vertices of the triangle.
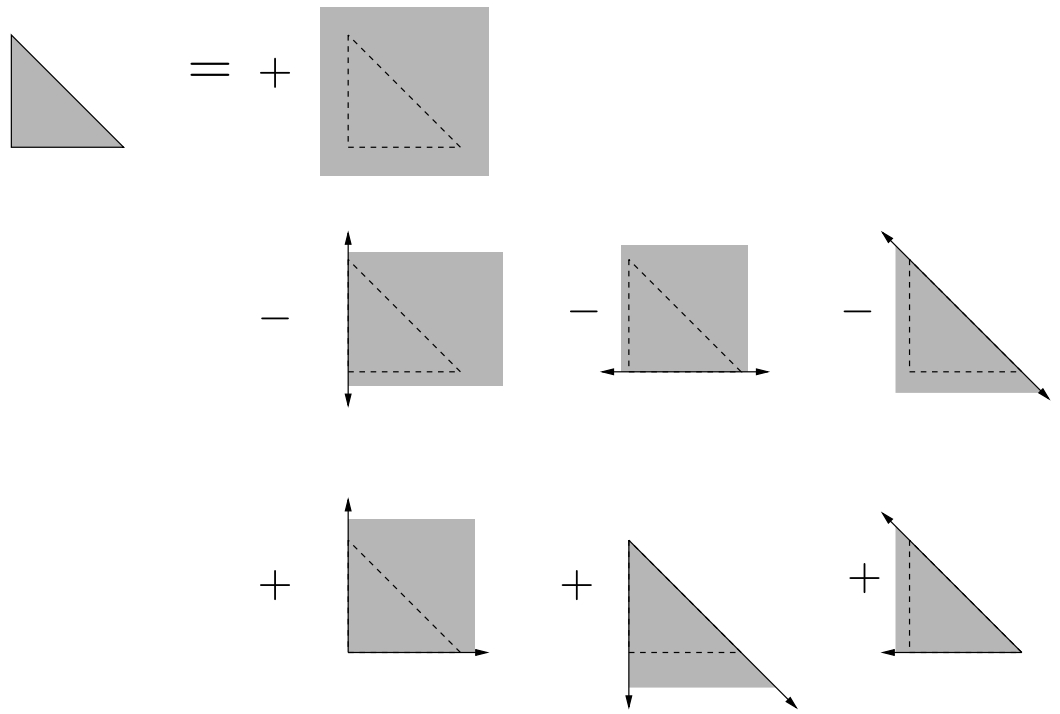

Figure 2: The Brianchon-Gram decomposition of a triangle. The summands on the right-hand side correspond to the faces of various dimensions of the triangle. 
tangent cone of $\Delta$ at a face $F$ is defined to be

$$
C_{F}=\left\{x+\lambda(y-x) \mid y \in \Delta, x \in F, \lambda \in \mathbb{R}_{\geq 0}\right\} .
$$

One may think of this as the polyhedral cone which "a near-sighted person would see" if she stands at a point in the relative interior of the face $F$. Clearly, $C_{F}$ is determined only by the local structure of $\Delta$ near this point. The (measure-theoretic version of the) Lawrence-Varchenko decomposition of $\Delta$ can then be expressed in the equality

$$
\mu_{\Delta}=\sum_{q}(-1)^{\epsilon_{q}} \mu_{C_{q}^{\sharp}}
$$

where the summation is over the vertices $q$ of $\Delta$, where $\mu_{\Delta}$ is Lebesgue measure on $\Delta$, where $\mu_{C_{q}^{\sharp}}$ is Lebesgue measure on the cone obtained from the tangent cone to $\Delta$ at $q$ by flipping some of its edge vectors so that they all pair positively with a pre-chosen "polarizing vector" in the dual space, and where $\epsilon_{q}$ is the number of edge vectors that are flipped. The formula (6.2) has a symplectic-geometric interpretation as follows. Suppose that $\Delta$ is Delzant; this is equivalent to the condition that $\Delta$ is the momentum polytope of a symplectic toric manifold $M$. (See [12] for the definition and basic facts of symplectic toric manifolds and Delzant polytopes.) The DH measure of $M$ is precisely $\mu_{\Delta}$. The fixed points for the torus action on $M$ exactly correspond to the vertices of the polytope $\Delta$ under the momentum map. For each fixed point $p$, the tangent space $T_{p} M$, with the symplectic form, torus action, and orientation induced from those of $M$, is the symplectic toric manifold corresponding to the tangent cone $C_{q}$ of $\Delta$ at the vertex $q$ corresponding to $p$. This tangent space is isomorphic to $\mathbb{C}^{n}$ with its standard symplectic form and with the torus acting by rotations of the coordinates. Flipping the symplectic form on some of the coordinates in $\mathbb{C}^{n}$ and flipping the corresponding summands in the formula for the momentum map yields a symplectic vector space $\left(T_{p} M\right)^{\sharp}$ with a torus action and with momentum image $C_{q}^{\sharp}$. Taking its DH measure with respect to its original orientation, which differs from the symplectic orientation if $\epsilon_{q}$ is odd (the DH measure is then negative), the measure-theoretic Lawrence-Varchenko decomposition (6.2) becomes the assertion that the $\mathrm{DH}$ measure of $M$ is equal to that of

$$
\bigsqcup_{p}\left(T_{p} M\right)^{\sharp} .
$$

In the spirit of this manuscript, this equality of DH measures can be deduced from the fact that $M$ is cobordant to $\bigsqcup_{p}\left(T_{p} M\right)^{\sharp}$ as Hamiltonian $T$-manifolds 
(with $T=\left(S^{1}\right)^{n}$ ) equipped with proper momentum maps [16, Chap. 4, Section 6].

We next recall the Brianchon-Gram polytope decomposition formula $[8$, 17]. Let $\Delta$ be a polytope. The Brianchon-Gram formula is the following relation between the characteristic functions of the polytope and of the tangent cones of its faces:

$$
\mathbf{1}_{\Delta}(x)=\sum_{F}(-1)^{\operatorname{dim}(F)} \mathbf{1}_{C_{F}}(x) .
$$

Note that the summation is now over all faces $F$ of $\Delta$ of all dimensions, in contrast to the Lawrence-Varchenko decomposition. The measure-theoretic version of this decomposition is

$$
\mu_{\Delta}=\sum_{F}(-1)^{\operatorname{dim} F} \mu_{C_{F}},
$$

where $\mu_{\Delta}$ is again Lebesgue measure on $\Delta$, and $\mu_{C_{F}}$ is Lebesgue measure on the tangent cone $C_{F}$. Again, the formula (6.4) has a symplectic-geometric interpretation as follows. Suppose again that $\Delta$ is Delzant and let $M$ be the corresponding symplectic toric manifold. The measure $\mu_{C_{F}}$ is then the $\mathrm{DH}$ measure of the symplectic toric manifold $M_{C_{F}}$ that corresponds to the tangent cone $C_{F}$. When $\operatorname{dim} F=\ell$, this symplectic toric manifold $M_{C_{F}}$ is isomorphic to $\left(S^{1} \times \mathbb{R}\right)^{\ell} \times \mathbb{C}^{n-\ell}$ with the standard symplectic form and with the torus acting by rotations of the $S^{1}$ factors and of the $\mathbb{C}$ factors. By flipping the symplectic form on the first $\ell$ components of this product, we get an oriented symplectic toric manifold which we denote by $M_{C_{F}}^{\sharp}$ whose momentum image is still $C_{F}$. The orientation arising from the symplectic form is consistent with the original orientation only if $\ell$ is even. By taking the $\mathrm{DH}$ measure of $M_{C_{F}}^{\sharp}$ with respect to the original orientation, (6.4) becomes the assertion that the DH measure of $M_{\Delta}$ is equal to that of $\bigsqcup_{F} M_{C_{F}}^{\sharp}$. We show below that this assertion coincides with our localization formula (4.25) when applied to the toric manifold $M$ with an appropriate taming map.

Throughout this section we work with an identification $\mathfrak{t} \cong \mathbb{R}^{n} \cong \mathfrak{t}^{*}$. Suppose as above that $\Delta$ is a Delzant polytope and let $M$ be the corresponding symplectic toric manifold. It turns out that the measure-theoretic formula (6.4) is what we obtain from Theorem 4.24 when applied to $M$ with a taming map that comes from a function that satisfies the conditions described in the following lemma. 
Lemma 6.5. Let $\Delta \subset \mathbb{R}^{n}$ be an $n$-dimensional simple polytope. Then there exists an open neighborhood $U$ of $\Delta$ in $\mathbb{R}^{n}$ and a smooth function $\rho: U \rightarrow \mathbb{R}$ with the following properties:

(1) For each face $F$ of $\Delta$, the restriction $\left.\rho\right|_{\text {rel-int }(F)}$ of $\rho$ to the relative interior of $F$ has a unique critical point $x_{F}$.

(2) Let $F$ be an $\ell$-dimensional face of $\Delta$, and let $x_{F}$ be the critical point of $\left.\rho\right|_{\text {rel-int }(F)}$. Then there exist $\varepsilon>0$ and affine coordinates $\left(x_{1}, \ldots, x_{\ell}\right.$, $\left.y_{1}, \ldots, y_{n-\ell}\right)$ on $\mathbb{R}^{n}$ with respect to which

(a) the point $x_{F}$ becomes the origin, and a neighborhood $x_{F}$ in $F$ becomes the set $U_{x_{F}}:=(-\varepsilon, \varepsilon)^{\ell} \times(-\varepsilon, 0]^{n-\ell}$ for some $\varepsilon>0$.

(b) The function $\left.\rho\right|_{U_{x_{F}}}$ becomes

$$
\rho\left(x_{1}, \ldots, x_{\ell}, y_{1}, \ldots, y_{n-\ell}\right)=\sum_{j=1}^{\ell} x_{j}^{2}+\sum_{j=1}^{n-\ell} y_{j}
$$

after composing it with an affine map of $\mathbb{R}$ (i.e., multiplying by a constant and adding a constant).

The proof of this lemma is technical and unrelated to the arguments in this section so we relegate it to an appendix. We prove (an equivalent version of) the lemma in Appendix 7.3 as Parts (A) and (C) of Proposition A.1.

Now suppose that $(M, \omega, \Phi)$ is a symplectic toric $T$-manifold with momentum polytope $\Delta=\Phi(M)$. Let $\rho$ be a function as specified in Lemma 6.5, and let $v=d(-\rho) \circ \Phi: M \rightarrow \mathfrak{t}$ be the taming map corresponding to $-\rho$. Let $Z=\left\{v^{\sharp}=0\right\}$ be the corresponding localizing set. We begin with the following observation.

\section{Lemma 6.6.}

$$
Z=\bigsqcup_{F} Z_{F},
$$

where the union is over all the faces $F$ of $\Delta$, and where $Z_{F}=\Phi^{-1}\left(\left\{x_{F}\right\}\right)$. Moreover, every $Z_{F}$ consists of exactly one T-orbit.

Proof. We have

(6.7) $Z=\operatorname{Crit}(\rho \circ \Phi)=\bigsqcup_{F}\left\{x \in M: \Phi(x)\right.$ is a critical point of $\left.\left.\rho\right|_{\text {rel-int }(F)}\right\}$.

Indeed, the first equality is the content of (2.22), and the second equality follows from Lemma 2.25, since the orbit type strata of a symplectic toric 
manifold are exactly the preimages of the relative interiors of the faces of its momentum polytope. By the construction of $\rho$, (specifically property (1) of Lemma 6.5), the term in the union (6.7) that corresponds to the face $F$ is exactly $\Phi^{-1}\left(\left\{x_{F}\right\}\right)$. Because in a toric manifold the momentum level sets are exactly the $T$-orbits, $\Phi^{-1}\left(\left\{x_{F}\right\}\right)$ is a $T$-orbit.

Our next task is to explicitly construct a neighborhood $U_{Z}$ of $Z$ and a $v$-polarized completion $\left(U_{Z}, \omega_{Z}, \Phi_{Z}\right)$ of $\left(U_{Z},\left.\omega\right|_{U_{Z}},\left.\Phi\right|_{U_{Z}}\right)$ relative to $Z$ for which an application of Theorem 4.24 and a concrete computation of the right-hand side of (4.25) for our choice of $\left(U_{Z}, \omega_{Z}, \Phi_{Z}\right)$ yield the measuretheoretic Brianchon-Gram formula. By Lemma 6.6, we can construct $U_{Z}$ as a disjoint union, over faces $F$, of neighborhoods $U_{F}$ of $Z_{F}$, and we can construct the polarized completion separately on each $U_{F}$. The following result is the main technical tool that we need:

Proposition 6.8. Let $(M, \omega, \Phi)$ be a compact connected symplectic toric manifold with momentum polytope $\Delta=\Phi(M)$. Let $\rho: \Delta \rightarrow \mathbb{R}$ be a smooth function as in Lemma 6.5, and let $v: M \rightarrow \mathfrak{t}$ be the taming map corresponding to $-\rho$. Let $F$ be a face of $\Delta$ and let

$$
C_{F}=\left\{x+\lambda(y-x) \mid y \in \Delta, x \in F, \lambda \in \mathbb{R}_{\geq 0}\right\}
$$

be the tangent cone of $\Delta$ at $F$. Let $Z_{F}$ be the component of the localizing set that corresponds to the face $F$ as in Lemma 6.6. Then there exist

- an arbitrarily small $T$-invariant tubular neighborhood $U_{F}$ of $Z_{F}$;

- a v-polarized completion $\left(U_{F}, \omega_{F}, \Phi_{F}\right)$ of $\left(U_{F},\left.\omega\right|_{U_{F}},\left.\Phi\right|_{U_{F}}\right)$; and

- an isomorphism of (oriented) Hamiltonian T-manifolds between $\left(U_{F}, \omega_{F}, \Phi_{F}\right)$ and the symplectic toric manifold $\left(M_{C_{F}}, \omega_{C_{F}}, \Phi_{C_{F}}\right)$ corresponding to $C_{F}$, which carries the orientation on $U_{F}$ to the symplectic orientation on $M_{C_{F}}$ if $\operatorname{dim} F$ is even and to the opposite of the symplectic orientation on $M_{C_{F}}$ if $\operatorname{dim} F$ is odd.

Proof. Let $\ell$ denote the dimension of $F$. By Lemma 6.5 we assume that the affine span of $F$ is $\mathbb{R}^{\ell} \times\{0\} \subseteq \mathbb{R}^{n}$, the critical point $x_{F}$ is the origin 0 , the polytope $\Delta$ coincides near $x_{F}$ with the sector $\mathbb{R}^{\ell} \times \mathbb{R}_{\leq 0}^{n-\ell}$, and the function $\rho$ near $x_{F}$ is of the form

$$
\rho\left(x_{1}, \ldots, x_{\ell}, y_{1}, \ldots, y_{n-\ell}\right)=\sum_{j=1}^{\ell} x_{j}^{2}+\sum_{j=1}^{n-\ell} y_{j}
$$


The tangent cone $C_{F}$ is the sector

$$
\left\{\left(x_{1}, \ldots, x_{\ell}, y_{1}, \ldots, y_{n-\ell}\right) \mid y_{1}, \ldots, y_{n-\ell} \leq 0\right\}
$$

The corresponding symplectic toric manifold is

$$
M_{C_{F}}=\left(S^{1} \times \mathbb{R}\right)^{\ell} \times \mathbb{C}^{n-\ell}
$$

where the torus $T \cong T^{\ell} \times T^{n-\ell}$ acts by rotating the $S^{1}$ coordinates and the $\mathbb{C}$ coordinates. The symplectic form on $M_{C_{F}}$, which we denote $\omega_{\text {std }}$, is the split form which is equal to $d \theta \wedge d t$ on every cylinder (parameterized as $\left.\left\{\left(\mathrm{e}^{\mathrm{i} \theta}, t\right)\right\}\right)$ and is standard on the $\mathbb{C}^{n-\ell}$ factor. The momentum map is

$$
\begin{aligned}
\Phi_{C_{F}} & \left(\left(\mathrm{e}^{\mathrm{i} \theta_{1}}, t_{1}\right), \ldots,\left(\mathrm{e}^{\mathrm{i} \theta_{\ell}}, t_{\ell}\right), z_{1}, \ldots, z_{n-\ell}\right) \\
& =\left(t_{1}, \ldots, t_{\ell},-\frac{\left|z_{1}\right|^{2}}{2}, \ldots,-\frac{\left|z_{n-\ell}\right|^{2}}{2}\right) .
\end{aligned}
$$

The local normal form theorem identifies a neighborhood $U_{F}$ of $Z_{F}$ in $M$ with the open subset

$$
\left(S^{1} \times(-\varepsilon, \varepsilon)\right)^{\ell} \times\left(D_{\varepsilon}^{2}\right)^{n-\ell}
$$

of $M_{C_{F}}$, for some $\varepsilon>0$. Here $D_{\varepsilon}^{2}$ is a disc with momentum image $(-\varepsilon, 0]$. Thus its radius is $\sqrt{2 \varepsilon}$.

By the explicit formula (6.9) for $\rho$, and identifying $\mathfrak{t}$ and $\mathfrak{t}^{*}$ with $\mathbb{R}^{n}$, if $\varepsilon$ is sufficiently small, the identification of $U_{F}$ with the open subset (6.10) of $M_{C_{F}}$ carries the taming map $v$ to the pullback via $\Phi_{C_{F}}$ of

$$
\left(x_{1}, \ldots, x_{\ell}, y_{1}, \ldots, y_{n-\ell}\right) \mapsto\left(-2 x_{1}, \ldots,-2 x_{\ell},-1, \ldots,-1\right)
$$

Denote this pullback $v_{\text {std }}$. The pairing of the momentum map $\Phi_{C_{F}}$ with the taming map $v_{\text {std }}$ is the function

$$
-\sum_{j=1}^{\ell} 2 t_{j}^{2}+\sum_{j=1}^{n-\ell} \frac{\left|z_{j}\right|^{2}}{2}
$$

which is neither proper nor bounded from below. Its restriction to the open subset (6.10) is bounded but is not proper.

We now equip $M_{C_{F}}$ with the split symplectic form, which we denote $\omega_{C_{F}}^{\sharp}$, which is the negative of $d \theta \wedge d t$ on every cylinder component and remains standard on the $\mathbb{C}^{n-\ell}$ component. This symplectic form $\omega_{C_{F}}^{\sharp}$ is consistent 
with the original symplectic orientation if $\ell$ is even and inconsistent if $\ell$ is odd, and it has the momentum map

$$
\Phi_{C_{F}}^{\sharp}=\left(-t_{1}, \ldots,-t_{\ell},-\frac{\left|z_{1}\right|^{2}}{2}, \ldots,-\frac{\left|z_{n-\ell}\right|^{2}}{2}\right) .
$$

We will now describe an equivariant diffeomorphism from $U_{F}$ to $M_{C_{F}}$ under which the pullbacks of $\omega_{C_{F}}^{\sharp}$ and $\Phi_{C_{F}}^{\sharp}$ coincide with $\omega$ and $\Phi$ on $Z_{F}$ (in fact, their further pullbacks to $Z_{F}$ are zero) and under which the pullback of $\Phi_{C_{F}}^{\sharp}$ is $v$-polarized.

Let $g:(-\varepsilon, \varepsilon) \rightarrow \mathbb{R}$ be a diffeomorphism such that $g(-x)=-g(x)$ for all $x$ and such that $g(x)=x$ on a neighborhood of $x=0$. Consider the diagram

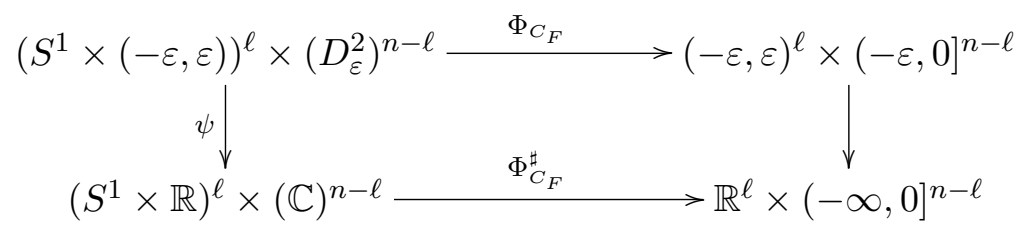

in which the right vertical map is

$$
\left(x_{1}, \ldots, x_{\ell}, y_{1}, \ldots, y_{n-\ell}\right) \mapsto\left(g\left(-x_{1}\right), \ldots, g\left(-x_{\ell}\right), g\left(y_{1}\right), \ldots, g\left(y_{n-\ell}\right)\right)
$$

and the left vertical map $\psi$ is the map that acts on the first $\ell$ factors as $\left(\mathrm{e}^{\mathrm{i} \theta_{k}}, x_{k}\right) \mapsto\left(\mathrm{e}^{\mathrm{i} \theta_{k}}, g\left(x_{k}\right)\right)$ and on the last $n-\ell$ coordinates by $z_{j}=r_{j} \mathrm{e}^{\mathrm{i} \theta_{j}} \mapsto$ $\sqrt{2 g\left(r_{j}^{2} / 2\right)} \mathrm{e}^{\mathrm{i} \theta_{j}}$. From this explicit description of $\psi$ it follows that $\psi$ is a $T$-equivariant diffeomorphism and that the diagram (6.12) commutes.

Let $\omega_{F}:=\psi^{*} \omega_{C_{F}}^{\sharp}$ and $\Phi_{F}:=\psi^{*} \Phi_{C_{F}}^{\sharp}$. The Hamiltonian $T$-manifold

$$
\left(U_{F}, \omega_{F}, \Phi_{F}\right)
$$

is isomorphic to $\left(M_{C_{F}}, \omega_{C_{F}}^{\sharp}, \Phi_{C_{F}}^{\sharp}\right)$ since the equivariant diffeomorphism $\psi$ provides such an isomorphism. To finish the proof we must show that $\Phi_{F}$ is $v$-polarized. Since the diagram (6.12) commutes, this is equivalent to showing that the composition of the top horizontal arrow with the right vertical arrow in (6.12) is $v$-polarized. Recall that the taming map $v$ is the pullback via $\Phi_{C_{F}}$ of (6.11). Since the momentum map $\Phi_{C_{F}}$, taken with the domain and codomain as in the top horizontal arrow of (6.12), is proper, it is enough to show that the pairing of the map (6.11) with the right vertical arrow 
in (6.12) is proper and bounded from below. This pairing is the map

$$
(-\varepsilon, \varepsilon)^{\ell} \times(-\varepsilon, 0]^{n-\ell} \rightarrow \mathbb{R}
$$

that is given by the formula

$$
\begin{aligned}
\left(x_{1}, \ldots, x_{\ell}, y_{1}, \ldots, y_{n-\ell}\right) \mapsto & \left(-2 x_{1}, \ldots,-2 x_{\ell},-1, \ldots,-1\right) \\
& \cdot\left(g\left(-x_{1}\right), \ldots, g\left(-x_{\ell}\right), g\left(y_{1}\right), \ldots, g\left(y_{n-\ell}\right)\right) \\
= & 2 \sum_{j=1}^{\ell} x_{j} g\left(x_{j}\right)-\sum_{j=1}^{n-\ell} g\left(y_{j}\right) .
\end{aligned}
$$

Notice that $y_{j}$ takes values in $(-\varepsilon, 0]$, and $-g\left(y_{j}\right)$ is nonnegative and approaches $\infty$ as $y_{j}$ approaches $-\varepsilon$. Also, $x_{j}$ takes values in $(-\varepsilon, \varepsilon)$, and $x_{j} g\left(x_{j}\right)$ is nonnegative and approaches $\infty$ as $\left|x_{j}\right| \rightarrow \pm \varepsilon$. So the function (6.13) is nonnegative, and for every $L$ there exists $\delta$ such that $0<\delta<\varepsilon$ and such that both $t g(t)$ and $g(t)$ are $\geq L$ whenever $\delta \leq|t|<\epsilon$, and so the preimage of $[0, L]$ under the function (6.13) is contained in the compact subset $[-\delta, \delta]^{\ell} \times[-\delta, 0]^{n-\ell}$ of the domain $(-\varepsilon, \varepsilon)^{\ell} \times(-\varepsilon, 0]^{n-\ell}$. This shows that the function is proper and bounded from below.

This proposition allows us to identify the left and right-hand sides of our localization formula (4.25), applied to the symplectic toric manifold $M$ and the taming map obtained from the function $\rho$, with the left and right-hand sides of the Brianchon-Gram decomposition (6.4), applied to the momentum polytope $\Delta$ of $M$. The right-hand side of our localization formula (4.25) is a summation over the components of the localizing set. These components exactly correspond to the faces $F$ of $\Delta$. By Proposition (6.8), a neighborhood of the component that corresponds to the face $F$ has a polarized completion that is isomorphic to the symplectic toric manifold $M_{C_{F}}$ with an orientation that is consistent with its symplectic form if and only if $\operatorname{dim} F$ is even. Thus, the localization formula (4.25) in this case becomes the equality

$$
\mathrm{DH}_{\mathrm{M}}=\sum_{\mathrm{F}}(-1)^{\operatorname{dim} \mathrm{F}} \mathrm{DH}_{\mathrm{M}_{\mathrm{C}_{\mathrm{F}}}} .
$$

Since the DH measure of a symplectic toric manifold is precisely Lebesgue measure on its momentum polytope, this equality is precisely the BrianchonGram equality (6.4). 
Remark 6.14. Our results are not the first to relate the Brianchon-Gram polytope decomposition to localization. A partial answer to Question 6.1 is given by localization theory using the norm-square $\|\Phi\|^{2}$ of a momentum map for a Hamiltonian G-space, as developed by Paradan [29] and Woodward [34], following Witten [33]. Indeed, the localization formula with respect to $\|\Phi\|^{2}$, applied to the exponent of the equivariant symplectic form of a symplectic toric manifold, yields the Brianchon-Gram decomposition under the following assumption on the momentum polytope $\Delta$ :

for every face $F$ of $\Delta$, of any dimension, the point of $F$ that is closest to the origin lies in the relative interior of $F$.

This correspondence between the localization formula for $\|\Phi\|^{2}$ and the Brianchon-Gram decomposition was also observed by Jonathan Weitsman and was worked out by Agapito and Godinho in [1]. Moreover, when the assumption (6.15) on $\Delta$ fails, Agapito and Godinho show that the localization formula for the norm-square of the momentum map corresponds to a new polytope decomposition that is different from Brianchon-Gram's.

We close by addressing the issue of the difference between the BrianchonGram formula and its measure-theoretic version.

Remark 6.16. Although the Brianchon-Gram formula (6.3) can be proved directly, it can also be derived from its measure-theoretic version, (6.4). Because the measures that appear in (6.4) are constant multiples of Lebesgue measure outside the union of the affine spans of the facets of $\Delta$, this measuretheoretic formula implies the formula (6.3) whenever $x$ is outside the union of these affine spans. To prove (6.3) for an arbitrary $x$, we apply the measuretheoretic formula (6.4) to the polytope that is obtained from $\Delta$ by shifting its facets outward by an amount (depending on $x$ ) that is small enough to not affect the values at $x$ of the left and right-hand sides of (6.3).

\section{Example: a circle action on the 2-sphere}

As an illustration of our methods, we now work out in detail the case of $S^{1}$ acting on the unit sphere $S^{2} \subset \mathbb{R}^{3}$ with the standard rotation action. We begin by setting some notation. The area form on $S^{2}$ can be written in cylindrical coordinates as $\omega=d \theta \wedge d h$ where $h: S^{2} \rightarrow \mathbb{R}$ is the height function; this equips $S^{2}$ with the standard orientation. The $S^{1}$ action is generated by the vector field $\partial / \partial \theta$. We identify the Lie algebra $\operatorname{Lie}\left(S^{1}\right)$ and 


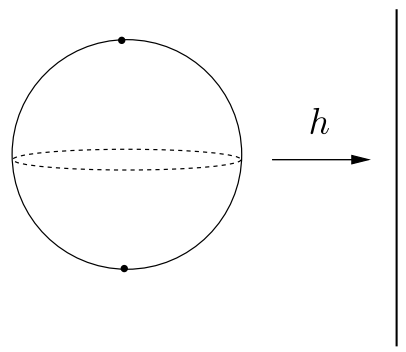

$\mathbb{R}$

Figure 3: The standard action of $S^{1}$ on $S^{2}$ has momentum map the height function $\Phi=h$. The equator has value $h=0$, the north pole has $h=1$, and the south pole has $h=-1$.

its dual $\operatorname{Lie}\left(S^{1}\right)^{*}$ with $\mathbb{R}$ so that the exponential map becomes $\theta \mapsto \mathrm{e}^{\mathrm{i} \theta}$. Then Hamilton's equation (2.17) becomes $d \Phi=\iota(\partial / \partial \theta) \omega$, and the momentum map can be given by the height function $\Phi=h$, as indicated in figure 3 .

Below, we apply our localization theorem to three different choices of taming map $v: S^{2} \rightarrow \operatorname{Lie}\left(S^{1}\right)^{*} \cong \mathbb{R}$, obtaining as a consequence three different polytope decompositions. The first example recovers the classical $\mathrm{DH}$ theorem and hence the measure-theoretic Lawrence-Varchenko decomposition. The second is the decomposition given by Woodward's localization with respect to $\|\Phi\|^{2}$, and finally, the third is the measure-theoretic BrianchonGram decomposition.

\subsection{Example: a constant taming map}

We first consider the case corresponding to Example 2.26, i.e., where the taming map $v$ is equal to a constant $\eta \in \operatorname{Lie}\left(S^{1}\right) \cong \mathbb{R}$. For concreteness we take $\eta=1$. In this case $Z=\left\{\eta^{\sharp}=0\right\}=\left(S^{2}\right)^{S^{1}}=\{N, S\}$, so the localizing set is the classical localizing set consisting of the fixed points of the action. An equivariant tubular neighborhood $U_{Z}$ of $Z$ consists of two components $U_{N}$ and $U_{S}$, equivariant neighborhoods of the north and south poles respectively. In order to apply Theorem 4.24 we must choose $v$-polarized completions of $(\Phi=h, \omega=d \theta \wedge d h)$ on both $U_{N}$ and $U_{S}$. We first consider the north pole $N$. By definition, a $v$-polarized completion $\left(U_{N}, \omega_{N}, \Phi_{N}\right)$ of $\left(\mathbb{N},\left.\omega\right|_{U_{N}},\left.\Phi\right|_{U_{N}}\right)$ must satisfy $\Phi_{N}(N)=\Phi(N)=h(N)=1$ and $\Phi_{N}^{v}=\Phi_{N}$ must be proper and bounded below. (There is no condition on $\omega_{N}$ because $\{N\}$ is 0 -dimensional so the restriction of any 2-form to that component of $Z$ is 0 .) 
In order to make explicit computations, we choose an orientationpreserving $S^{1}$-equivariant diffeomorphism (not symplectomorphism) from an open neighborhood $U_{N}$ of $N$ to (all of) $\mathbb{C}$, equipped with its standard orientation and $S^{1}$-action. The momentum map for the standard symplectic form on $\mathbb{C}$ is $-\frac{1}{2}\|z\|^{2}$ (up to a constant), which is not bounded below. To correct this, we therefore equip $\mathbb{C}$ with the negative of the standard symplectic form, $-\omega_{\text {std }}=-d x \wedge d y$, and we take the momentum map

$$
\Phi_{N}(z)=1+\frac{1}{2}\|z\|^{2}
$$

which is both bounded below and proper. Hence, we can take $\left(U_{N}, \omega_{N}, \Phi_{N}\right)$ to be given by $(7.1)$ and $\omega_{N}=-\omega_{\text {std }}$. Because integration of $-\omega_{\text {std }}=-d x \wedge$ $d y$ with respect to the standard orientation takes negative values, the corresponding DH measure is negative Lebesgue measure on the ray $[1, \infty)$ and zero outside the ray.

Similarly, a neighborhood $U_{S}$ of the south pole can be identified via an orientation-preserving $S^{1}$-equivariant diffeomorphism with $\mathbb{C}$ with its standard orientation and the opposite $S^{1}$-action:

$$
\lambda: z \mapsto \lambda^{-1} z
$$

The momentum map for this action is, up to a constant, $+\frac{1}{2}\|z\|^{2}$, which is already proper and bounded below. To obtain the condition $\Phi_{S}(S)=$ $\Phi(S)=h(S)=-1$ we define

$$
\Phi_{S}(z)=-1+\frac{1}{2}\|z\|^{2}
$$

Here we take the standard symplectic form (and not its negative), so the contribution from the south pole is positive Lebesgue measure on the ray $[-1, \infty)$ and zero outside.

Hence we get the decomposition of the Duistermaat-Heckman measure of $S^{2}$ as illustrated in the following figure 4 . This corresponds to the Lawrence-Varchenko polytope decomposition of the interval $[-1,1]$.

\subsection{Example: the norm-square of the momentum map}

We now consider the case corresponding to Example 2.27, i.e., where $v=$ $\widehat{\Phi}=h$. In this case, the zero set $Z:=\left\{x \in S^{2}: v_{x}^{\sharp}=0\right\}$ of $v^{\sharp}$ is $\{N\} \cup\{S\} \cup$ $\{h=0\}$, so we have an additional component of $Z$ corresponding to the equator in $S^{2}$. 


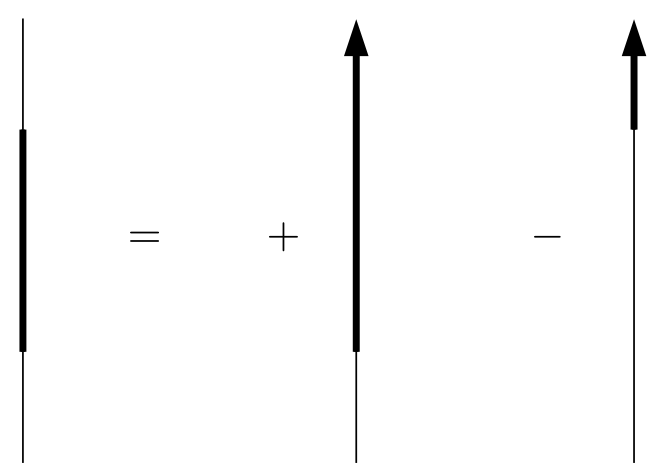

Figure 4: By choosing $v \equiv 1$ constant and applying the localization formula, we obtain the Lebesgue measure on the interval $[-1,1]$ as the difference of Lebesgue measures on two rays. This corresponds to a Lawrence-Varchenko decomposition.

We begin our computations with the north pole. As in the previous example, we must construct a $v$-polarized completion $\left(\Phi_{N}, \omega_{N}\right)$ of $\left(\left.\Phi\right|_{U_{N}},\left.\omega\right|_{U_{N}}\right)$ on $U_{N}$. We may assume that $U_{N}$ is contained in the upper quarter of the sphere, $\{1 / 2<h \leq 1\}$, so $\Phi_{N}^{v}$ is between $\frac{1}{2} \Phi_{N}$ and $\Phi_{N}$. So $\Phi_{N}^{v}$ is proper and bounded from below if and only if $\Phi_{N}$ is proper and bounded from below. The same analysis as in the previous example applies and we take

$$
\Phi_{N}(z)=1+\frac{1}{2}\|z\|^{2}
$$

with $U_{N} \cong \mathbb{C}$ equipped with the negative of the standard symplectic form. Hence the contribution from the north pole is the negative Lebesgue measure on $[1, \infty)$, as in the previous example.

In the case of the south pole, however, the analysis is different from the previous example since we now have $v \approx-1<0$ near the south pole. We may assume that $U_{S}$ is contained in the bottom quarter of the sphere, $\{-1 \leq h \leq-1 / 2\}$. Thus, in order to satisfy the $v$-polarization condition, we must construct $\Phi_{S}$ such that its negative $-\Phi_{S}$ is proper and bounded from below. A similar analysis as in the previous case then shows that we may take the negative of the standard symplectic form on $\mathbb{C}$ and momentum map

$$
\Phi_{S}(z)=-1-\frac{1}{2}\|z\|^{2}
$$

with contribution negative Lebesgue measure on the ray $(-\infty,-1]$. 


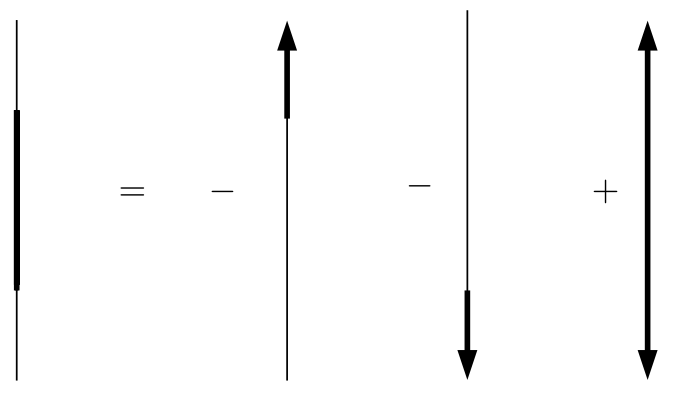

Figure 5: By choosing $v=\hat{\Phi}=h$, we obtain the Lebesgue measure on the interval $[-1,1]$ as the sum of three contributions as indicated. This corresponds to localization via the norm-square of the momentum map.

We now consider the contribution from the equator. The neighborhood $\left\{-\frac{1}{4}<h<\frac{1}{4}\right\}$ of the equator in $S^{2}$ is (non-symplectically) equivariantly diffeomorphic to the cylinder $S^{1} \times \mathbb{R}$, with coordinates $(\theta, s)$. The action of $S^{1}$ is by standard multiplication on the left component of $S^{1} \times \mathbb{R}$. By Hamilton's equation (2.17), and using the standard orientation of the cylinder given by the symplectic form $\omega_{E}=d \theta \wedge d s$, the momentum map $\Phi_{E}$ is given by $\Phi_{E}(\theta, s)=s$, i.e., projection onto the second factor. This momentum map satisfies $\left.\Phi_{E}\right|_{s=0}=0$, so it agrees with the height function at the equator, as required. Moreover, when $s \gg 0$ we have $v \approx \frac{1}{4}$, and when $s \ll 0$ we have $v \approx-\frac{1}{4}$. So we have $\Phi_{E}^{v}(\theta, s) \approx \frac{1}{4} s$ for $s \gg 0$ and $\Phi_{E}^{v}(\theta, s) \approx\left(-\frac{1}{4}\right) s$ for $s \ll 0$. So $\Phi_{E}^{v}(\theta, s) \approx \frac{1}{4}|s|$ for $|s| \gg 0$, and hence $\Phi_{E}$ is also $v$-polarized. The orientation of $\omega_{E}$ is the same as the orientation induced from the standard orientation of $S^{2}$ restricted to $U_{E}$, so this term will appear with no sign change. Hence the contribution from the equator is positive Lebesgue measure on all of $\mathbb{R}$.

In summary, we get that the DH measure for the $S^{1}$-action on $S^{2}$ may be written in terms of these three contributions, as given in figure 5 . This is the decomposition corresponding to the localization via the norm-square of the momentum map.

\subsection{Example: the negative of the norm-square of the momentum map}

Finally, we consider the example where we pick $v=-\widehat{\Phi}=-h$. Since $S^{2}$ is compact, $\Phi^{v}=-h^{2}$ is bounded below so $\Phi=h$ is $v$-polarized on $S^{2}$. We have $Z=\{N\} \cup\{S\} \cup\{h=0\}$ as in the previous case. The analysis of the components of an equivariant tubular neighborhood $U_{Z}=U_{N} \cup U_{S} \cup U_{E}$ is 


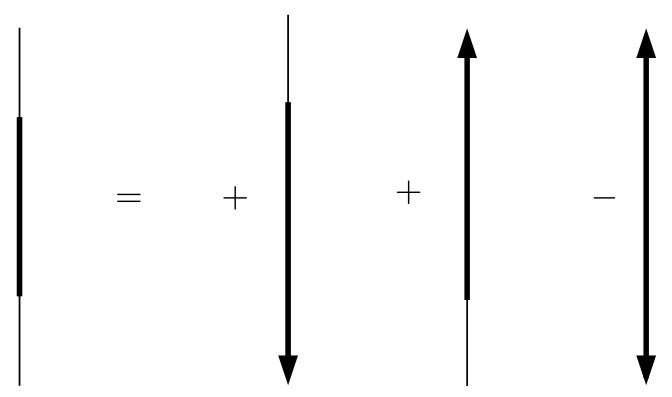

Figure 6: By choosing $v=-\hat{\Phi}=-h$, we obtain the Lebesgue measure on the interval as the sum of three contributions as indicated. This corresponds to the Brianchon-Gram decomposition.

exactly analogous to the previous case and we do not go through the details here, and only note that the choices of direction will differ because of the sign change in $v$.

This choice of $v$ yields the decomposition of the $\mathrm{DH}$ measure as illustrated in figure 6. This is the Brianchon-Gram decomposition.

\section{Acknowledgments}

It is our pleasure to thank Shlomo Sternberg and Jonathan Weitsman for inspiring questions and conversations. We thank both Victor Guillemin and Shlomo Sternberg for their interest and encouragement in this project. We thank Brendan McLellan and Lisa Jeffrey for useful discussions, Chris Woodward for explaining his results to us, and Michèle Vergne for helping to clarify Witten's work. We also thank the two anonymous referees, who read our manuscript very carefully and made many helpful suggestions for improving exposition. Both authors are partially supported by NSERC Discovery Grants. The first author is additionally partially supported by an NSERC University Faculty Award and an Ontario Ministry of Research and Innovation Early Researcher Award.

\section{Appendix A. Construction on a simple polytope of a smooth function with a unique critical point on the relative interior of each face}

In this appendix, we prove the technical Proposition A.1, which asserts the existence of a smooth function on a simple compact polytope $\Delta$ with certain prescribed properties, the most important of which is that it has a unique 
critical point on the relative interior of every face. The existence of a function with such critical points is intuitively quite clear but, firstly, we could not find a reference, and secondly, it is surprisingly difficult to prove rigorously. Our approach is to use brute-force differential topology on $\mathbb{R}^{n}$. In fact, our explicit construction yields a function which, near a critical point in a face $F$, is linear in coordinates transverse to the face, and quadratic in coordinates along the face. These specific properties of our construction are used in Section 6. Moreover, although we do not explicitly use these properties in this manuscript, we can also specify in advance the location and the function value of each of the critical points in the faces of $\Delta$.

We begin with some terminology and notation. By a polytope $\Delta$ we mean the convex hull of a finite set of points in a vector space (or in an affine space). In particular, our polytopes are always compact. A face $F$ of $\Delta$ is its intersection with a supporting hyperplane: $F=\Delta \cap\{L=\lambda\}$ where $L$ is a linear functional and $\left.L\right|_{\Delta} \geq \lambda$. The dimension of a polytope is the dimension of its affine span. Faces of a polytope are themselves polytopes. Facets are faces of codimension one. Every face is an intersection of facets. A polytope is simple if every face of codimension $k$ is contained in no more than $k$ facets. Given a convex subset $X$ of $\mathbb{R}^{n}$, we denote by rel-int $(X)$ the relative interior of $X$, i.e., the interior of $X$ in the affine span of $X$. Finally, we recall that a function $f$ defined on an arbitrary subset of $X$ of $\mathbb{R}^{n}$ (e.g., on a polytope) is said to be smooth if, near each point $x \in X$, there exists a smooth extension $f_{U}$ of $f$ to an open neighborhood $U$ of $x$ such that $\left.f_{U}\right|_{U \cap X}=\left.f\right|_{U \cap X}$. In the case that $X$ is closed as a subset of $\mathbb{R}^{n}$, it then follows that $f$ extends to a global smooth function on all of $\mathbb{R}^{n}$.

The following technical proposition records the results of our explicit construction:

Proposition A.1. Let $\Delta \subset \mathbb{R}^{n}$ be an $n$-dimensional simple polytope. Then there exists a smooth function $f: \Delta \rightarrow \mathbb{R}$ with the following properties.

(A) For every face $F$ of $\Delta$, the minimal value of $f$ on $F$ is attained at exactly one point in the relative interior of $F$, and the restriction of $f$ to the relative interior of $F$ has no other critical points.

(B) Suppose that we are given the data $\left\{\left(x_{F}, \alpha_{F}\right): F\right.$ a face in $\left.\Delta\right\}$, where for each face $F$ we have $x_{F} \in \operatorname{rel}-\operatorname{int}(F)$ and $\alpha_{F} \in \mathbb{R}_{\geq 0}$, and where $\alpha_{F}<\alpha_{F^{\prime}}$ whenever $F^{\prime}$ is a proper subface of $F$. Then the function $f$ may be chosen such that, for each face $F$ of $\Delta$, the restriction $\left.f\right|_{\text {rel-int }(F)}$ attains its minimum at the chosen point $x_{F}$, and the minimum value is the chosen $\alpha_{F}$, i.e., $f\left(x_{F}\right)=\alpha_{F}$. 
(C) Let $F$ be an $\ell$-dimensional face of $\Delta$. Near the above given point $x_{F} \in$ $F$, there exist $\varepsilon>0$, a neighborhood $U_{x_{F}}$ of $x_{F}$ in $F$, and affine local coordinates $\left(x_{1}, \ldots, x_{\ell}, y_{1}, \ldots, y_{n-\ell}\right) \in(-\varepsilon, \varepsilon)^{n}$ on $U_{x_{F}}$ such that

(a) the neighborhood $U_{F}$ is given by $\left\{\left(x_{1}, \ldots, x_{\ell}, y_{1}, \ldots, y_{n-\ell}\right) \in\right.$ $\left.(-\varepsilon, \varepsilon)^{\ell} \times[0, \varepsilon)^{n-\ell}\right\}$ and the point $x_{F}$ is given by the origin $(0,0) \in$ $(-\varepsilon, \varepsilon)^{\ell} \times[0, \varepsilon)^{n-\ell}$

(b) the function $f$ can be chosen such that with respect to these coordinates, $\left.f\right|_{U_{x_{F}}}$ is of the form

$$
f\left(x_{1}, \ldots, x_{\ell}, y_{1}, \ldots, y_{n-\ell}\right)=\sum_{j=1}^{\ell} x_{j}^{2}-\sum_{j=1}^{n-\ell} y_{j}
$$

up to an affine translation in $\mathbb{R}$ (i.e., up to a multiplication and translation by constants).

In order to prove Proposition A.1, we construct the required function $f$ by a recursive procedure. Before delving into technicalities, we first sketch our method. Recall that the $\ell$-skeleton of a polytope $\Delta$ is the union of its $\ell$-dimensional faces. We begin the inductive argument by constructing an appropriate function $f_{0}$ on a neighborhood in $\mathbb{R}^{n}$ of the 0 -skeleton, i.e., of the vertices of $\Delta$. Such a function will automatically have a unique critical point on the relative interior of each vertex, because these relative interiors are just single points. Continuing by induction, suppose that we are given a function $f_{\ell-1}$, satisfying appropriate technical conditions (to be specified below) on a neighborhood in $\mathbb{R}^{n}$ of the $(\ell-1)$-skeleton. After possibly shrinking this neighborhood, we show that there exists a function $f_{\ell}$ on a neighborhood of the $\ell$-skeleton extending $f_{\ell-1}$ and satisfying similar technical conditions. Continuing in this manner, at the final step we then obtain a function $f:=$ $f_{n}$, defined on all of $\Delta$ and that has the desired properties. The concrete implementation of this plan occupies the rest of this appendix.

At each inductive step the functions $f_{\ell}$ that we construct are required to satisfy conditions that are stated in terms of certain vector fields $\xi_{i}$ that are defined along the facets of $\Delta$, point "into" the interior of $\Delta$, and are tangent to lower-dimensional faces. We therefore begin with the construction of these vector fields, for which we need some notation. Let $\Delta$ be a simple polytope in $\mathbb{R}^{n}$ with $N$ facets. We may express the polytope as an intersection of half-spaces, i.e., $\Delta=\bigcap_{i=1}^{N} H_{i}$ where

$$
H_{i}=\left\{x \in \mathbb{R}^{n} \mid \phi_{i}(x) \leq \lambda_{i}\right\}
$$


the $\phi_{i}$ are linear functionals on $\mathbb{R}^{n}$, and the $\lambda_{i}$ are real numbers. We always assume that $\Delta$ has non-empty interior in $\mathbb{R}^{n}$. Let $\left\{\sigma_{1}, \sigma_{2}, \ldots, \sigma_{N}\right\}$ be the facets of $\Delta$, i.e., $\sigma_{i}=\Delta \cap \partial H_{i}$. For a subset $I \subseteq\{1,2, \ldots, N\}$, let $F_{I}$ denote the (possibly empty) face of $\Delta$ obtained by intersecting the facets $\sigma_{i}$ for $i \in I$ :

$$
F_{I}:=\bigcap_{i \in I} \sigma_{i} \subseteq \Delta .
$$

We define $F_{\emptyset}:=\Delta$. If $F_{I}$ is nonempty, then since $\Delta$ is simple, $F_{I}$ is a face of codimension $|I|$. Moreover, if $J \subset I$ then $F_{I} \subset F_{J}$.

With this terminology in place we construct the vector fields $\xi_{j}$ along the facets which we use throughout our construction. Identifying $\left.T \mathbb{R}^{n}\right|_{\sigma_{j}}$ with $\sigma_{j} \times \mathbb{R}^{n}$ in the standard way, we think of these vector fields as functions $\xi_{j}: \sigma_{j} \rightarrow \mathbb{R}^{n}$.

Lemma A.3. Let $\Delta=\bigcap_{i=1}^{N}\left\{x \in \mathbb{R}^{n} \mid \phi_{i}(x) \leq \lambda_{i}\right\}$ be an $n$-dimensional simple polytope in $\mathbb{R}^{n}$ and let $\left\{\sigma_{i}\right\}_{i=1}^{N}$ denote the facets of $\Delta$. Then there exist smooth vector fields $\xi_{j}: \sigma_{j} \rightarrow \mathbb{R}^{n}$ along the facets such that

(1) $d \phi_{j}\left(\left.\xi_{j}\right|_{x}\right)<0$ for all $x \in \sigma_{j}$, and

(2) $d \phi_{i}\left(\left.\xi_{j}\right|_{x}\right)=0$ for all $x \in \sigma_{j} \cap \sigma_{i}$ and $i \neq j$.

In particular, let $F=\sigma_{j_{1}} \cap \cdots \cap \sigma_{j_{n-\ell}}$ and let

$$
\pi=\left(\lambda_{j_{1}}-\phi_{j_{1}}, \ldots, \lambda_{j_{n-\ell}}-\phi_{j_{n-\ell}}\right): \mathbb{R}^{n} \rightarrow \mathbb{R}^{n-\ell}
$$

Then for all $x \in F$ the vectors $\left\{\pi_{*}\left(\left.\xi_{j_{1}}\right|_{x}\right), \ldots, \pi_{*}\left(\left.\xi_{j_{n-\ell}}\right|_{x}\right)\right\}$ are positive multiples of the standard basis elements of $\mathbb{R}^{n-\ell}$.

Moreover, having a priori chosen for each face $F$ a point $x_{F}$ in the relative interior of $F$, the $\xi_{j}$ can be chosen to be constant on a neighborhood of $x_{F}$ for each $F$.

Condition (1) in Lemma A.3 means that $\left.\xi_{j}\right|_{x}$ is transverse to $\sigma_{j}$ and points into $\Delta$. Condition (2) implies that, for any face $F_{I}$ and for the indices $j$ that correspond to relative facets $F_{I} \cap \sigma_{j}$, the restrictions of the vector fields $\xi_{j}$ to $F_{I} \cap \sigma_{j}$ are tangent to $F_{I}$ and point into $F_{I}$.

Proof. Let $x \in \Delta \backslash \operatorname{rel-int}(\Delta)$. Let $j_{1}, j_{2}, \ldots, j_{n-\ell} \in\{1,2, \ldots, N\}$ be the indices of all the facets that pass through $x$. The linear functionals $\phi_{j_{1}}, \ldots, \phi_{j_{n-\ell}}$ are linearly independent in $\left(\mathbb{R}^{n}\right)^{*}$ since the polytope is simple. Let $t_{1}, \ldots, t_{\ell}$ be linear functionals such that $t_{1}, \ldots, t_{\ell}, \phi_{j_{1}}, \ldots, \phi_{j_{n-\ell}}$ is a basis of $\left(\mathbb{R}^{n}\right)^{*}$. Let $t_{\ell+1}:=\lambda_{j_{1}}-\phi_{j_{1}}, \ldots, t_{n}:=\lambda_{j_{n-\ell}}-\phi_{j_{n-\ell}}$. Then there exists a 
neighborhood $U_{x}$ of $x$ in $\Delta$ such that $t_{1}, \ldots, t_{n}: \mathbb{R}^{n} \rightarrow \mathbb{R}^{n}$ carries $U_{x}$ onto a neighborhood of 0 in $\mathbb{R}^{\ell} \times \mathbb{R}_{\geq 0}^{n-\ell}$. Note in particular that for every point $y$ of $U_{x}$, the indices $j_{1}, j_{2}, \ldots, j_{n-\ell} \in\{1,2, \ldots, N\}$ are precisely the indices of all the facets that pass through $y$. of $U_{x}$.

The vectors $\xi_{i}^{(x)}:=\frac{\partial}{\partial t_{\ell+i}}$ satisfy the required properties at all the points

Now fix $j \in\{1,2, \ldots, N\}$ and consider $\sigma_{j}$. The open sets $\left\{U_{x} \cap \sigma_{j}\right\}_{x \in \sigma_{j}}$ form an open cover of $\sigma_{j}$. Since $\Delta$ is compact, so is $\sigma_{j}$, so we may choose a finite subcover $\left\{U_{x_{s}} \cap \sigma_{j}\right\}_{s=1}^{N_{j}}$ for some $N_{j} \in \mathbb{N}$. Without loss of generality we may assume that for each $F$ contained in $\sigma_{j}$, the point $x_{F}$ appears among the $\left\{x_{s}\right\}_{s=1}^{N_{j}}$. We may also assume that for each $x_{F}$, there is a sufficiently small neighborhood $V_{F} \subseteq U_{x_{F}}$ such that $U_{x_{s}} \cap V_{F}=\emptyset$ for all $x_{s} \neq x_{F}$. Let $\rho_{s}: \sigma_{j} \rightarrow \mathbb{R}, 1 \leq s \leq N_{j}$, be a smooth partition of unity with supp $\rho_{s} \subseteq U_{x_{s}} \cap$ $\sigma_{j}$. Define

$$
\xi_{j}:=\sum_{s} \rho_{s} \xi_{j}^{\left(x_{s}\right)} .
$$

Then the vector fields $\xi_{1}, \ldots, \xi_{N}$ satisfy the required properties.

Using the above vector fields $\xi_{j}$, we may now state the recursive conditions on the functions $f_{\ell}$ in our construction:

(f1) $f_{\ell}$ is a smooth function defined on an open neighborhood $U_{\ell}$ in $\Delta$ of the $\ell$-skeleton;

(f2) for each face $F$ of dimension $\leq \ell$, the restriction of $f_{\ell}$ to $\operatorname{rel}-\operatorname{int}(F)$ attains its minimum at the point $x_{F}$, has no critical points other than $x_{F}$, and $f_{\ell}\left(x_{F}\right)=\alpha_{F} ;$ and

(f3) for each face $F$ of dimension $\leq \ell$, we have $d f_{\ell}\left(\left.\xi_{j}\right|_{x}\right)<0$ for all $j$ with $\sigma_{j} \cap F \neq \emptyset$ and for all $x \in \sigma_{j} \cap F$.

(Note that $\sigma_{j} \cap F$ can be either $F$ itself or a relative facet of $F$.)

We now begin the recursive construction of the functions $f_{\ell}$. The base case requires us to construct a function $f_{0}$ near the 0 -skeleton satisfying (f1)-(f3) above. Let $x$ be a vertex of $\Delta$. Since $\Delta$ is simple, there exists an open neighborhood $U_{x} \subset \mathbb{R}^{n}$ of $x$ and an element $A$ of $\operatorname{AGL}(n, \mathbb{R})$ (=affine automorphisms of $\mathbb{R}^{n}$ ) such that the map $A$ takes $x$ to 0 and takes the intersection $U_{x} \cap \Delta$ to a neighborhood of 0 in the positive orthant $\mathbb{R}_{\geq 0}^{n}=$ $\left\{v=\left(v_{1}, \ldots, v_{n}\right) \in \mathbb{R}^{n}: v_{i} \geq 0\right.$ for all $\left.1 \leq i \leq n\right\}$. We may take $\left.f_{0}\right|_{U_{x}}$ to be the composition of the affine transformation $A$ with the function $v \mapsto \alpha_{F}-$ $\sum_{i} v_{i}$. The last condition in Lemma A.3 implies that for each facet $\sigma_{j}$ that contains the vertex $x$ of $\Delta$ the vector field $\xi_{i}$, near $x$, is a positive multiple of 
$\frac{\partial}{\partial v_{i}}$ in the above coordinates for $\mathbb{R}_{\geq 0}^{n}$. Hence the function $f_{0}$ near $x$ satisfies the condition (f3) above. By our formula for $\left.f_{0}\right|_{U_{x}}$, the condition (f2) also holds at this vertex. Repeating this for all vertices (and possibly shrinking the open neighborhoods so that their closures are disjoint), we obtain a function that satisfies the above conditions (f1)-(f3) with $\ell=0$. This completes the base case of the induction.

We now proceed with the recursive step. Let $\ell \geq 1$, and assume that we have already defined a function $f_{\ell-1}$ on a neighborhood of the $(\ell-1)$ skeleton that satisfies the conditions (f1)-(f3). We now construct a function $f_{\ell}$ which (after possibly shrinking the neighborhood on which $f_{\ell-1}$ is defined) extends $f_{\ell-1}$ near each $\ell$-dimensional face $F$ separately. In fact, we will first construct $f_{\ell}$ in the $\ell$-dimensional affine span of $F$ and then extend to a neighborhood of $F$ in $\mathbb{R}^{n}$. Fix an $\ell$-dimensional face $F$ of $\Delta$. By using an affine change of coordinates, we may assume without loss of generality that

- the affine span of $F$ is $\mathbb{R}^{\ell}$, identified with the subspace $\mathbb{R}^{\ell} \times\{0\}^{n-\ell}$ of $\mathbb{R}^{n}$;

- the chosen point $x_{F} \in \operatorname{rel-int} F$ is the origin 0 ; and

- in a neighborhood of 0 the polytope $\Delta$ coincides with $\mathbb{R}^{\ell} \times \mathbb{R}_{\geq 0}^{n-\ell}$.

Furthermore, after permuting the indices if necessary, we may without loss of generality assume that $F=\sigma_{1} \cap \cdots \cap \sigma_{n-\ell}$, so that the vector fields $\xi_{1}, \ldots, \xi_{n-\ell}$ are defined along $F$.

The set of indices

$$
\mathcal{K}:=\left\{k \mid \sigma_{k} \cap F \neq \emptyset, n-\ell<k \leq N\right\}
$$

parameterizes the set of relative facets of $F$. We have on each relative facet $\sigma_{k} \cap F$ a vector field $\xi_{k}$ along which $f_{\ell-1}$ decreases. In Lemma A.4 below we construct a single vector field $\eta^{F}$ on a neighborhood $V_{F} \backslash\{0\}$ of $F \backslash\{0\}$ in $\mathbb{R}^{\ell}$ which interpolates between $-\frac{\partial}{\partial r}$ and the vector fields $\xi_{j}$ constructed in Lemma A.3, and along which $f_{\ell-1}$ decreases where it is defined. The flow along $\eta^{F}$ allows us to reparameterize the relative interior of $F$, which will in turn allow us to explicitly construct the extension $f_{\ell}$ of $f_{\ell-1}$ near $F$.

Let $r$ denote the radial coordinate in $\mathbb{R}^{\ell}$ and let $-\frac{\partial}{\partial r}$ denote the corresponding inward-pointing radial vector field, defined and smooth on $\mathbb{R}^{\ell}$ $\{0\}$. Let $k \in \mathcal{K}$. Denote by $L_{k}$ the restriction to the affine subspace $\mathbb{R}^{\ell}$ of the linear functional $\frac{1}{\lambda_{k}} \phi_{k}$ in the notation of (A.2). Then the face $F$ as a subset 
of $\mathbb{R}^{\ell}$ is given by the intersection

$$
F=\bigcap_{k \in \mathcal{K}}\left\{x \in \mathbb{R}^{\ell} \mid L_{k}(x) \leq 1\right\} \subseteq \mathbb{R}^{\ell} .
$$

Given $\delta>0$, we may also define a smaller polytope $F_{\delta} \subseteq F$ by

$$
F_{\delta}:=\bigcap_{k \in \mathcal{K}}\left\{x \in \mathbb{R}^{\ell} \mid L_{k}(x) \leq 1-\delta\right\} \subseteq \mathbb{R}^{\ell} .
$$

We always assume that $\delta$ is sufficiently small such that $F_{\delta}$ has the same combinatorial type as $F$ (this is possible because $\Delta$ is simple) and such that $0 \in$ rel-int $F_{\delta}$.

Lemma A.4. There exists an open subset $V_{F}$ of $\mathbb{R}^{\ell}$ containing $F$, a smooth vector field $\eta^{F}$ on $V_{F} \backslash\{0\}$, and a constant $\delta, 0<\delta<1$, such that

(1) for all $k \in \mathcal{K}$,

$$
d L_{k}\left(\eta^{F}\right)<-\delta<0 \quad \text { on } \quad L_{k}^{-1}([1-\delta, 1]) \cap V_{F}
$$

(2) $\eta^{F}=-\frac{\partial}{\partial r}$ on $F_{\delta} \backslash\{0\}$; and

(3) $d f_{\ell-1}\left(\eta^{F}\right)<0$ on $\partial F$.

Proof. The proof is by explicit construction. We first construct vector fields locally which satisfy the conditions of the lemma, and then patch them together using a partition of unity.

We begin with the interior of $F$. Consider the open set $U_{0}:=\operatorname{rel}-\operatorname{int}(F) \backslash$ $\{0\}$. The radial vector field $\eta_{0}:=-\frac{\partial}{\partial r}$ is certainly smooth on $U_{0}$. Moreover, since $0 \in \operatorname{rel-int}(F)$ and $F$ is convex, $-\frac{\partial}{\partial r}$ is transverse to all relative facets $F_{k}=\sigma_{k} \cap F$ of $F$. In particular, for each $k \in \mathcal{K}$ we have $d L_{k}\left(\eta_{0}\right)<0$ on $F_{k}$. Since each $F_{k}$ is compact, there exists a neighborhood $V_{k}$ of $F_{k}$ and $\delta_{k}>0$ such that $d L_{k}\left(\eta_{0}\right)<-\delta_{k}<0$ on $V_{k}$. Let $\delta_{k}^{\prime}>0$ be such that $L_{k}^{-1}([1-$ $\left.\left.\delta_{k}^{\prime}, 1\right]\right) \cap U_{0} \subseteq V_{k}$ for each $k$, and set $\delta_{0}:=\min \left(\cup_{k \in \mathcal{K}}\left\{\delta_{k}, \delta_{k}^{\prime}\right\}\right)$. Then, for all $k \in \mathcal{K}$, we have

$$
d L_{k}\left(\eta_{0}\right)<-\delta_{0} \quad \text { on } \quad L_{k}^{-1}\left(\left[1-\delta_{0}, 1\right]\right) \cap U_{0}
$$

We conclude that the vector field $\eta_{0}$ on $U_{0}$ satisfies the conditions (1) and (2) of the lemma. (Since $U_{0}$ does not intersect $\partial F$, the condition (3) is not relevant for this case.) 
We now proceed to the local construction at the boundary. Let $x$ be in the relative boundary $\partial F$ of $F$ in $\mathbb{R}^{\ell}$. Let $\mathcal{K}_{x}:=\left\{k \in \mathcal{K} \mid x \in F_{k}\right\}$. By Lemma A.3(2), the vectors $\left.\xi_{j}\right|_{x}$ for $j \in \mathcal{K}_{x}$ lie in $\mathbb{R}^{\ell}$. Let

$$
\eta_{x}:=\left.\sum_{j \in \mathcal{K}_{x}} \xi_{j}\right|_{x}
$$

Observe that by the property (f3) of $f_{\ell-1}$ and the definition of the $\eta_{x}$,

$$
d f_{\ell-1}\left(\eta_{x}\right)<0
$$

Moreover, by Lemma A.3(1), the derivative $d L_{k}\left(\eta_{x}\right)$ is negative for every $k \in \mathcal{K}_{x}$, since $d L_{k}\left(\xi_{k}\right)<0$ and $d L_{k}\left(\xi_{j}\right)=0$ for all $j \in \mathcal{K}_{x} \backslash\{k\}$. Choose $\delta_{x}^{\prime}$ such that $0<\delta_{x}^{\prime}<\min \left\{-d L_{k}\left(\eta_{x}\right)\right\}_{k \in \mathcal{K}_{x}}$. We now deal with the indices $s$ not in $\mathcal{K}_{x}$. By the definition of $\mathcal{K}_{x}, L_{s}(x)<1$ for all $s \in \mathcal{K} \backslash \mathcal{K}_{x}$. Choose $\delta_{x}^{\prime \prime}$ such that $0<\delta_{x}^{\prime \prime}<\min \left\{1-L_{s}(x)\right\}_{s \in \mathcal{K} \backslash \mathcal{K}_{x}}$. Then $x \notin L_{s}^{-1}\left(\left[1-\delta_{x}^{\prime \prime}, 1\right]\right)$ for all $s \in \mathcal{K} \backslash \mathcal{K}_{s}$. Let $U_{x}^{\prime}$ be a neighborhood of $x$ in $\mathbb{R}^{\ell}$ such that $0 \notin U_{x}^{\prime}$, $U_{x}^{\prime} \cap L_{s}^{-1}\left(\left[1-\delta_{x}^{\prime \prime}, 1\right]\right)=\emptyset$ for all $s \in \mathcal{K} \backslash \mathcal{K}_{x}$, and $d\left(f_{\ell-1}\right)_{y}\left(\eta_{x}\right)(y)<0$ at all points $y$ of $U_{x}^{\prime}$. (Here $\eta_{x}$ is viewed as a vector field on $U_{x}$ with constant coefficients.) Since the relative boundary $\partial F$ of $F$ is compact, there exists a finite set $\left\{x_{1}, \ldots, x_{m}\right\} \subseteq \partial F$ such that $\partial F \subseteq U_{x_{1}}^{\prime} \cup U_{x_{2}}^{\prime} \cup \cdots \cup U_{x_{m}}^{\prime}$. Let $\delta=\min \left\{\delta_{x_{1}}^{\prime}, \delta_{x_{1}}^{\prime \prime}, \ldots, \delta_{x_{m}}^{\prime}, \delta_{x_{m}}^{\prime \prime}\right\}$. Define $V_{F}:=U_{0} \cup U_{x_{1}}^{\prime} \cup \ldots \cup U_{x_{m}}^{\prime}$. Then, by construction, $V_{F}$ contains $F$, and the sets $U_{0}, U_{x_{1}}^{\prime} \backslash\left(U_{x_{1}}^{\prime} \cap F_{\delta}\right), \ldots, U_{x_{m}}^{\prime} \backslash$ $\left(U_{x_{m}}^{\prime} \cap F_{\delta}\right)$ form an open covering of $V_{F}$. Let $\rho_{0}, \rho_{1}, \ldots, \rho_{m}$ be a partition of unity subordinate to this covering, and let

$$
\eta^{F}:=\rho_{0}\left(-\frac{\partial}{\partial r}\right)+\sum_{i=1}^{m} \rho_{i} \eta_{x_{i}} .
$$

Then $\eta^{F}, V_{F}$, and $\delta>0$, as chosen above, satisfy the conditions of the lemma.

We now wish to show that we may use the vector field $\eta^{F}$ constructed in Lemma A.4 to reparameterize the relative interior of $F$. Denote by $\psi(t, x)$ the flow along $-\eta^{F}$, where $t$ is the time parameter of the flow and $x$ is the initial condition. For fixed initial condition $x$, by a maximal trajectory through $x$ we mean the flow $\psi(t, x)$ on the maximal interval $(a, b) \subseteq \mathbb{R}$ on which the flow is defined. As a first step, we wish to show that for any $x$ in $\partial F_{\delta}$, this flow $\psi(t, x)$ takes $x$ to $\partial F$ in finite time:

Lemma A.8. Let $\eta^{F}$ and $\delta$ be as in Lemma A.4 and let $x$ be a point in $\partial F_{\delta}$. Let $X(t):=\psi(t, x)$ be the maximal trajectory of $-\eta^{F}$ with initial 
condition $\psi(0, x)=x$. Then there exists $t_{x}>0$ such that $X(t) \in \operatorname{rel}-i n t F$ for all $0 \leq t<t_{x}$ and $X\left(t_{x}\right) \in \partial F$.

Proof. Since $x \in \partial F_{\delta}$, there exists $k \in \mathcal{K}$ such that $L_{k}(x)=1-\delta$. Fix one such $k$. Let $t>0$. First we claim that

if $X(\tau)$ is defined and takes values in $F$ for all $\tau$ in $[0, t]$ then

$$
L_{k}(X(t))>1-\delta+\delta t
$$

To prove the claim, recall that by the construction of $\eta^{F}$ and $\delta$, if $X(t)$ is defined and belongs to $F$, then

(A.10) if $\quad L_{k}(X(t)) \geq 1-\delta \quad$ then $\frac{d}{d t} L_{k}(X(t))=\left(d L_{k}\right)_{X(t)}\left(-\eta^{F}\right)>\delta$.

Since $X(0)=x$ and $L_{k}(x)=1-\delta$, then by (A.10) we may conclude that the derivative $\frac{d}{d t} L_{k}(X(t))$ is greater than $\delta$ when $t=0$. By continuity, this derivative is greater than $\delta$ for $t$ in a neighborhood of 0 . Integrating, we conclude that (A.9) is true if $t$ is positive and sufficiently small. Observe also that by continuity of solutions of ordinary differential equations, for any $t>0$ for which (A.9) holds, there exists an open interval containing $t$ on which the trajectory is defined and for which (A.9) still holds; in particular, the set of $t$ for which the claim holds is open. Now suppose that there exists a positive $t_{0}$ for which the claim does not hold; then there exists a minimal such $t_{0}$ since the set of $t_{0}$ for which the claim does not hold is closed. By minimality, if $0<t<t_{0}$ then $L_{k}(X(t))>1-\delta+\delta t$. By (A.10), $L_{k}(X(t))>1-\delta+\delta t$ and $t>0$ imply $\frac{d}{d t} L_{k}(X(t))>\delta$. Integrating,

$$
\begin{aligned}
L_{k}\left(X\left(t_{0}\right)\right) & =L_{k}(X(0))+\int_{0}^{t_{0}} \frac{d}{d t} L_{k}(X(t)) d t>L_{k}(X(0))+\int_{0}^{t_{0}} \delta d t \\
& =1-\delta+\delta t_{0},
\end{aligned}
$$

which contradicts the assumption on $t_{0}$. This completes the proof that (A.9) holds for all positive $t$.

Now suppose that the assertion of the lemma is not true, that is, $X(t) \in$ rel-int $F$ for all $t>0$ where $X(t)$ is defined. Then (A.9) implies that $X(t) \notin$ $F_{\delta}$ for all $t>0$ where $X(t)$ is defined. This implies that $X(t)$ is defined for all $t \in[0, \infty)$, because it coincides with the trajectory of a compactly supported vector field that equals $\eta_{F}$ on $F \backslash F_{\delta}$. Taking $t$ big enough so that $1-\delta+\delta t$ is greater than one, (A.9) contradicts the assumption that $X(t)$ is in $F$. This proves the lemma. 
We may now use $\psi(t, x)$ to reparameterize $F$. In order to do so smoothly, we first need to construct a smooth manifold in $F$ which approximates $\partial F$ in $F$. We will construct this smooth approximation by taking a regular level set of a smooth function, which we now construct:

Lemma A.11. There exists a smooth function $h^{F}$ on a neighborhood of $\partial F$ in $F$ such that

- $d h^{F}\left(\eta^{F}\right)<0$ at all points of $\operatorname{rel-int}(F)$ where $h^{F}$ is defined, and

- $\left.h^{F}\right|_{\partial F} \equiv 1$.

Proof. We first construct a function satisfying the conditions of the lemma locally near any point $x \in \partial F$. We then use a partition of unity constant along $\eta^{F}$ to form the global function $h^{F}$ required in the lemma.

Let $x \in \partial F$ and let $U_{x}$ be a neighborhood of $x$ in $\mathbb{R}^{\ell}$ such that $U_{x}$ only intersects facets of $F$ that contain $x$ and such that $U_{x}$ is contained in the set $V_{F}$ where $\eta^{F}$ is defined. By construction, $\left(d L_{k}\right)_{x}\left(\eta^{F}\right)<0$ for all $k \in \mathcal{K}_{x}$, so after possibly shrinking $U_{x}$, we may assume that $d L_{k}\left(\eta^{F}\right)<0$ at all points in $U_{x}$. On this neighborhood $U_{x}$, we define the function

$$
h^{x}:=1-\prod_{k \in \mathcal{K}_{x}}\left(1-L_{k}\right) .
$$

At points $y \in U_{x} \cap \partial F$, at least one of the $L_{k}$ in the right-hand side of (A.12) is equal to one, so $h^{x}(y)=1$. At points $y \in U_{x} \cap$ rel-int $F$, all the $L_{k}$ in (A.12) are less than 1 , so $h^{x}(y)<1$. Since $d L_{k}\left(\eta^{F}\right)<0$ for all $k \in \mathcal{K}_{x}$, each $L_{k}$ is decreasing along the trajectories of $\eta^{F}$. From (A.12) we then see that $h^{x}$ is also decreasing along $\eta^{F}$ at any point $y$ where $L_{k}(y)<1$ for all $k \in \mathcal{K}$. In particular, $d h^{x}\left(\eta^{F}\right)<0$ on $U_{x} \cap \operatorname{rel}$-int $F$.

Secondly, we patch together these functions $h^{x}$ by means of a partition of unity. This will require some extra care since we wish to guarantee that the resulting function still satisfies the first condition of the lemma. To accomplish this, we now construct a partition of unity $\left\{\rho^{x}\right\}$ such that for each $\rho^{x}$ we have $d \rho^{x}\left(\eta^{F}\right)=0$, i.e., the functions $\rho^{x}$ are constant along the flow of $\eta^{F}$. Let $x \in \partial F$. Since $\left.\eta^{F}\right|_{x} \neq 0$ and $\eta^{F}$ is smooth, there exists a neighborhood $V_{x} \subset U_{x}$ of $x$ in $\mathbb{R}^{\ell}$ with a smooth parametrization $\phi: \Omega_{x} \rightarrow$ $V_{x}$, where

$$
\Omega_{x}:=\left\{\left(t_{1}, \ldots, t_{\ell}\right) \in \mathbb{R}^{\ell}|| t_{1} \mid<4 \varepsilon_{1} \text { and } t_{2}^{2}+\cdots+t_{\ell}^{2}<4 \varepsilon_{2}\right\}
$$


for some $\varepsilon_{1}, \varepsilon_{2}>0$, such that $\phi(0)=x$ and $\phi_{*}\left(\frac{\partial}{\partial t_{1}}\right)=\eta^{F}$. Since $\eta^{F}$ is transverse to $\partial F$, after possibly shrinking $\varepsilon_{1}$ and $\varepsilon_{2}$, we may assume without loss of generality that for every value of $t_{2}, \ldots, t_{\ell}$ that occurs in $\Omega_{x}$, the function $t_{1} \mapsto L_{k}\left(\phi\left(\left(t_{1}, t_{2}, \ldots, t_{\ell}\right)\right)\right.$ is defined for $t_{1}$ in some interval, is strictly monotone, and assumes both positive and negative values. It then follows that for every $\left(t_{2}, \ldots, t_{\ell}\right)$ occurring in $\Omega_{x}$, there exists exactly one $t_{1}$ such that $\phi\left(t_{1}, t_{2}, \ldots, t_{\ell}\right) \in \partial F$. Moreover, after possibly further shrinking $\varepsilon_{2}$, we may also assume that

$$
\left\{\left(t_{1}, t_{2}, \ldots, t_{\ell}\right) \mid \phi\left(t_{1}, \ldots, t_{\ell}\right) \in \partial F\right\}
$$

is contained in $\left\{\left|t_{1}\right|<2 \varepsilon_{1}\right\}$.

Now observe that the function

$\rho^{x}(q):= \begin{cases}\mathrm{e}^{-\frac{1}{\varepsilon_{2}^{2}-t_{2}^{2}-\cdots-t_{\ell}^{2}}} & \text { if } q=\phi\left(t_{1}, t_{2}, \ldots, t_{\ell}\right) \text { for some }\left(t_{1}, t_{2}, \ldots, t_{\ell}\right) \in \Omega_{x} \\ 0 & t_{2}^{2}+\cdots+t_{\ell}^{2}<\varepsilon_{2}, \text { and }\left|t_{1}\right|<\frac{5}{2} \varepsilon_{1} \\ 0 & \text { otherwise }\end{cases}$

is smooth on the neighborhood

$$
\widetilde{V}_{x}:=\mathbb{R}^{\ell} \backslash\left\{\phi\left(t_{1}, t_{2}, \ldots, t_{\ell}\right)\left|2 \varepsilon_{1} \leq\right| t_{1} \mid \leq 3 \varepsilon_{1}, \quad t_{2}^{2}+\cdots+t_{\ell}^{2} \leq 2 \varepsilon_{2}\right\}
$$

of $\partial F$ in $\mathbb{R}^{\ell}$. To see this, observe that $\widetilde{V}_{x}$ is the union of the open set $\left\{\phi\left(t_{1}, \ldots, t_{\ell}\right)|| t_{1} \mid<2 \varepsilon_{1}\right\}$ and the open set $\mathbb{R}^{\ell} \backslash\left\{\phi\left(t_{1}, \ldots, t_{\ell}\right)|| t_{1} \mid \leq 3 \varepsilon_{1}\right.$, $\left.t_{2}^{2}+\ldots+t_{\ell}^{2} \leq 2 \varepsilon_{2}\right\}$. On the second set, $\rho^{x} \equiv 0$ by definition. On the first set, $\rho^{x}$ is smooth. Hence $\rho^{x}$ is smooth also on the union.

By slight abuse of notation, we let $\left\{\rho^{x}>0\right\}$ denote the open subset in $\widetilde{V}_{x}$ where $\rho^{x}$ is positive. By definition of $\rho^{x}$, the point $x \in \partial F$ is contained in $\left\{\rho^{x}>0\right\}$. Since $\partial F$ is compact, there exists a finite set $\left\{x_{1}, \ldots, x_{M}\right\} \subseteq \partial F$ such that $\cup_{j=1}^{M}\left\{\rho^{x_{j}}>0\right\}$ contains $\partial F$. In particular $\sum_{j=1}^{M} \rho^{x_{j}}$ is defined and positive on a neighborhood of $\partial F$ in $\mathbb{R}^{\ell}$. Let $\widetilde{V}$ be such a neighborhood. We now define

$$
\rho^{k}:=\frac{1}{\sum_{j=1}^{M} \rho^{x_{j}}} \rho^{x_{k}}
$$

on $\widetilde{V} \cap \widetilde{V}_{x_{k}}$. Since $d \rho^{x_{k}}\left(\eta^{F}\right) \equiv 0$ by construction, it follows that $d \rho^{k}\left(\eta^{F}\right) \equiv 0$ also.

Since $h^{x_{k}}$ is defined on $U_{x_{k}}$ which entirely contains the support of $\rho^{x_{k}}$, the product $\rho^{k} h^{x_{k}}$ may be extended to a smooth function on all of $\widetilde{V}$. Hence 
the sum

$$
h^{F}:=\sum_{i=1}^{M} \rho^{i} h^{x_{i}}
$$

is well-defined and smooth on $\widetilde{V}$.

We now claim that $h^{F} \equiv 1$ on $\partial F$ and $d h^{F}\left(\eta^{F}\right)<0$ on $\widetilde{V} \cap \operatorname{rel-int} F$. To see this, suppose $x \in \partial F$. Let $\mathcal{I}_{x}=\left\{k \mid x \in U_{x_{k}}\right\}$. Consider

$$
\left(\bigcap_{k \in \mathcal{I}_{x}} U_{x_{k}}\right) \cap \tilde{V}
$$

On this set,

$$
h^{F}=\sum_{k \in \mathcal{I}_{x}} \rho^{k} h^{x_{k}}
$$

the $h^{x_{k}}$ for $k \in \mathcal{I}_{x}$ are well-defined, satisfy $d h^{x_{k}}\left(\eta^{F}\right)<0$ on $\operatorname{rel-int}(F)$, and moreover, $\sum_{k \in \mathcal{I}_{x}} \rho^{k} \equiv 1$. Hence for every $y$ in $\partial F \cap\left(\cap_{k \in \mathcal{I}_{x}} U_{x_{k}}\right) \cap \widetilde{V}$ we have

$$
h^{F}(y)=\sum_{k \in \mathcal{I}_{x}} \rho^{k}(y) h^{x_{k}}(y)=\sum_{k \in \mathcal{I}_{x}} \rho^{k}(y)=1 .
$$

Moreover, on rel-int $(F) \cap\left(\cap_{k \in \mathcal{I}_{x}} U_{x_{k}}\right) \cap \tilde{V}$, we have

$$
d h^{F}\left(\eta^{F}\right)=\sum_{k \in \mathcal{I}_{x}} d\left(\rho^{k} h^{x_{k}}\right)\left(\eta^{F}\right)=\sum_{k \in \mathcal{I}_{x}} \rho^{k} d h^{x_{k}}\left(\eta^{F}\right)<0
$$

where the second equality uses that $d \rho^{k}\left(\eta^{F}\right)=0$ by our construction of the $\rho^{k}$, and the last inequality uses that $d h^{x_{k}}\left(\eta^{F}\right)<0$, that $\rho^{k} \geq 0$, and that not all the $\rho^{k}$ vanish. Hence $h^{F}$ satisfies the required conditions of the lemma.

By the construction of $h^{F}$ in Lemma A.11, the differential $d h^{F}$ never vanishes in rel-int $F$. Thus, for any $\varepsilon>0$, the level set

$$
Z_{\varepsilon}:=\left(h^{F}\right)^{-1}(1-\varepsilon) \subset F
$$

is a smooth manifold. This is our smooth approximation to $\partial F$. The next lemma proves smoothness properties of the flow $\psi(t, x)$ along $-\eta^{F}$ with respect to this level set. Using (similar) notation as in Lemma A.8, for $x \in$ rel-int $F_{\delta} \backslash\{0\}$ we let $X(t)$ denote the trajectory for $-\eta_{F}$ that is defined for 
$0 \leq t \leq t_{x}$ and such that $X(0)=x, X\left(t_{x}\right) \in \partial F$, and $X(t) \in \operatorname{rel-int} F \backslash\{0\}$ for all $0<t<t_{x}$. Such a trajectory and $t_{x}$ exist because $\eta^{F}$ coincides with $-\frac{\partial}{\partial r}$ within $F_{\delta}$ and by Lemma A.8.

Lemma A.17. Let $\delta>0$ be as in Lemma A.4. Then there exists $\varepsilon>0$ sufficiently small such that for all $x \in$ rel-int $F_{\delta}$ there exists a unique $t_{x}^{\prime}>$ 0 with $h^{F}\left(X\left(t_{x}^{\prime}\right)\right)=1-\varepsilon$ and such that the function $x \mapsto t_{x}^{\prime}$ is smooth on rel-int $F_{\delta} \backslash\{0\}$.

Proof. Let $V$ be an open neighborhood of $\partial F$ in $F$ whose closure is contained in the open neighborhood of $\partial F$ where $h^{F}$ is defined. Its boundary, $\bar{V} \backslash V$, is a compact subset of rel-int $F$ on which $h^{F}<1$. Choose $\varepsilon$ such that $1-\varepsilon>\left.\max h^{F}\right|_{\bar{V} \backslash V}$. The existence of $t_{x}^{\prime}$ (specified by this choice of $\varepsilon)$ follows from the continuity of the function $t \mapsto h^{F}(X(t))$. The uniqueness of $t_{x}^{\prime}$ follows from the fact that $d h^{F}\left(-\eta^{F}\right)>0$ at all points of rel-int $F$ where $h^{F}$ is defined, which implies that $t \mapsto h^{F}(X(t))$ is monotone increasing.

The derivative $\left.\frac{\partial}{\partial t} h^{F} \circ \psi\right|_{(t, x)}$ is non-zero because it is equal to $d h_{\psi(t, x)}^{F}\left(-\eta^{F}\right)$, which is positive by the construction of $h^{F}$. The smoothness of the function $x \mapsto t_{x}^{\prime}$ now follows by applying the implicit function theorem to the condition $h^{F}(\psi(t, x))=1-\varepsilon$.

We now wish to use the flow along $-\eta^{F}$ to reparameterize the compact region in $F$ with boundary $Z_{\varepsilon}$ so that the region is diffeomorphically identified with the standard closed ball in $\mathbb{R}^{\ell}$ of some radius. Moreover, we want to arrange that under this identification, the vector field $\eta^{F}$ is identified with the standard inward-pointing radial vector field $-\frac{\partial}{\partial r}$. Such a parametrization allows us to construct the function $f_{\ell}$ using explicit coordinates on the standard closed ball.

We now review some properties of $f_{\ell-1}$ useful in the constructions to follow.

- First, $\min _{\partial F} f_{\ell-1}>\alpha_{F}$. Indeed, the induction hypotheses on $f_{\ell-1}$ imply that $\min _{\partial F} f_{\ell-1}=\min _{E \subsetneq F}\left\{\alpha_{E}\right\}$, which is greater than $\alpha_{F}$ by assumption.

- Second, $d f_{\ell-1}\left(\eta^{F}\right)<0$ on $\partial F$ by the construction of $\eta_{F}$ in Lemma A.4.

After possibly shrinking the neighborhood $U_{\ell-1}$ of $\partial F$ on which $f_{\ell-1}$ is defined, we may also assume without loss of generality that 
- $\inf _{U_{\ell-1} \cap \mathbb{R}^{\ell}} f_{\ell-1}>\alpha_{F}$;

- $\eta_{F}$ is well defined everywhere on $U_{\ell-1} \cap \mathbb{R}^{\ell}$; and

- $d f_{\ell-1}\left(\eta^{F}\right)<0$ on $U_{\ell-1} \cap \mathbb{R}^{\ell}$.

Let $\varepsilon>0$ be sufficiently small such that the conclusions of Lemma A.17 apply, such that the level set $Z_{\varepsilon}$ of (A.16) is contained in $U_{\ell-1}$, and such that $\alpha_{F}<\min _{Z_{\varepsilon}} f_{\ell-1}$. In particular, by the properties of $f_{\ell-1}$ listed above,

$$
d f_{\ell-1}\left(\eta^{F}\right)<0 \text { near } Z_{\varepsilon}
$$

To achieve the reparameterization mentioned above, we will need to further rescale $\eta^{F}$. Specifically, choose $r_{2}>r_{1}>0$ such that the set $F_{\delta}$ of Lemma A.4 contains the closed ball of radius $r_{2}$ about the origin, and consider the spheres $S_{r_{1}}^{\ell}$ and $S_{r_{2}}^{\ell}$ with center 0 and radii $r_{1}$ and $r_{2}$ respectively. In the lemma below and in the arguments that follow, we rescale $\eta^{F}$ within the region of $F$ contained between the concentric spheres $S_{r_{1}}^{\ell-1}$ and $S_{r_{2}}^{\ell-1}$. This makes the time required to flow out to $Z_{\varepsilon}$ uniform along $S_{r_{1}}^{\ell-1}$.

Lemma A.18. There exists a smooth function $\sigma: \mathbb{R}^{\ell} \rightarrow \mathbb{R}$ that takes positive values, is equal to 1 outside the set $\left\{x \in \mathbb{R}^{\ell}\left|r_{1}<\right| x \mid<r_{2}\right\}$, and such that for the flow $\psi(t, x)^{\text {new }}$ corresponding to the rescaled vector field $-\eta_{\text {new }}^{F}:=$ $-\sigma \eta^{F}$, there exists a constant $R>0$ such that $\psi(R, x)^{\text {new }}$ is defined and belongs to $Z_{\varepsilon}$ for all $x \in S_{r_{1}}^{\ell-1}$.

Proof. By construction, $\eta^{F}$ agrees with $-\frac{\partial}{\partial r}$ in the region between $S_{r_{1}}^{\ell-1}$ and $S_{r_{2}}^{\ell-1}$. Given a smooth function $h_{T}(r)$ of one variable and vector field $h_{T}(r) \frac{\partial}{\partial r}$ on $\mathbb{R}^{\ell} \backslash\{0\}$, it takes time

$$
\int_{r_{1}}^{r_{2}} \frac{d r}{h_{T}(r)}
$$

to flow along this vector field from a point in $S_{r_{1}}^{\ell-1}$ to $S_{r_{2}}^{\ell-1}$. Let $\beta:\left[r_{1}, r_{2}\right] \rightarrow$ $\mathbb{R}_{\geq 0}$ be a smooth function such that $\beta(r) \equiv 0$ for $r$ near both $r_{1}$ and $r_{2}$ and such that $\int_{r_{1}}^{r_{2}} \beta(r) d r=1$. For $T>0$, define $h_{T}:\left[r_{1}, r_{2}\right] \rightarrow \mathbb{R}$ by

$$
h_{T}(r):=\frac{1}{1+T \beta(r)} .
$$

Then by construction, the travel time (A.19) is equal to $r_{2}-r_{1}+T, h_{T}(r) \equiv$ 1 for $r$ near both $r_{1}$ and $r_{2}$, and $(T, r) \mapsto h_{T}(r)$ is smooth. 
Now, let $T: S_{r_{1}}^{\ell-1} \rightarrow \mathbb{R}_{>0}$ be a smooth function. For $x \in \mathbb{R}^{\ell}$, define

$$
\sigma(x)= \begin{cases}h_{T\left(r_{1} \frac{x}{\|x\|}\right)}(\|x\|) & \text { if } r_{1} \leq\|x\| \leq r_{2} \\ 1 & 0 \leq\|x\|<r_{1} \text { or }\|x\|>r_{2}\end{cases}
$$

This defines a smooth function $\sigma: \mathbb{R}^{\ell} \rightarrow \mathbb{R}$. Define a new vector field $\eta_{\text {new }}^{F}$ by

$$
\eta_{\text {new }}^{F}=\sigma \eta^{F}
$$

This vector field has the same trajectories as $\eta^{F}$, but with different time parameterizations. By the construction of the function $h_{T}$, the travel time along the trajectory from a point $x$ in $S_{r_{1}}^{\ell-1}$ to the sphere $S_{r_{2}}^{\ell-1}$ for the rescaled vector field $-\eta_{\text {new }}^{F}$ is equal to

$$
T(x)+r_{2}-r_{1}=T(x)+\left(\text { the travel time for the vector field }-\eta^{F}\right) .
$$

It follows that the travel time along the trajectory of $-\eta_{\text {new }}^{F}$ from a point $x \in S_{r_{1}}^{\ell-1}$ to $Z_{\varepsilon}$ is equal to $t_{x}^{\prime}+T(x)$ where $t_{x}^{\prime}$ is as in Lemma A.17. To finish the proof, it therefore remains to choose the function $T: S_{r_{1}}^{\ell-1} \rightarrow \mathbb{R}_{>0}$ so that $R:=t_{x}^{\prime}+T(x)$ is independent of $x \in S_{r_{1}}^{\ell-1}$. Pick any $R>\max _{x \in S_{r_{1}}^{\ell-1}} t_{x}^{\prime}$. For $x \in S_{r_{1}}^{\ell-1}$, define $T(x):=R-t_{x}^{\prime}$. Then this function $T$ is smooth, it takes positive values, and $T(x)+t_{x}^{\prime}$ is evidently independent of $x \in S_{r_{1}}^{\ell-1}$, as required.

We can use the modified $\eta_{\text {new }}^{F}$ to create a diffeomorphism between a standard closed ball in $\mathbb{R}^{\ell}$ and a subset of $F$ as follows. Let $\psi^{\text {new }}(t, x)$ denote the flow along $-\eta_{\text {new }}^{F}$. For a real number $r>0$, let $B^{\ell}(r, 0)$ denote the standard closed ball in $\mathbb{R}^{\ell}$ of radius $r$ centered at $0 \in \mathbb{R}^{\ell}$. We define

$$
\Psi: B^{\ell}\left(r_{1}+R, 0\right) \rightarrow F \backslash\left(h^{F}\right)^{-1}((1-\varepsilon, 1])
$$

by

$$
\Psi(x)= \begin{cases}x & \text { if }\|x\|<r_{1} \\ \psi^{\text {new }}(t, x) & \text { if } x=y+t \frac{y}{\|y\|} \text { for some } y \in S_{r_{1}}^{\ell-1} \\ & \text { and } 0 \leq t \leq R\end{cases}
$$

Lemma A.21. (1) $\Psi$ intertwines the vector field $-\frac{\partial}{\partial r}$ with the vector field $\eta_{\text {new }}^{F}$.

(2) $\Psi$ is a diffeomorphism. 
Proof. Since $\eta_{\text {new }}^{F}$ is defined in a neighborhood $V_{F}$ of $F$, the flow along the vector field $-\eta_{\text {new }}^{F}$ defines a smooth map $\left(-r_{1}, R+\varepsilon_{0}\right) \times S_{r_{1}}^{\ell-1} \rightarrow \mathbb{R}^{\ell}$, $(x, t) \mapsto \psi^{\text {new }}(t, x)$, for sufficiently small $\varepsilon_{0}>0$. We use the diffeomorphism $x+t \frac{x}{\|x\|} \mapsto(t, x)$ to identify the interior of $B\left(r_{1}+R+\varepsilon_{0}, 0\right) \backslash\{0\}$ with $\left(-r_{1}, R+\varepsilon_{0}\right) \times S_{r_{1}}^{\ell-1}$. Since $-\eta_{\text {new }}^{F} \equiv \frac{\partial}{\partial r}$ is the standard radial vector field within the ball of radius $r_{1}$, the restriction of this flow to $B^{\ell}\left(r_{1}+R, 0\right) \backslash\{0\}$ is precisely the map $\Psi$. In particular, $\Psi$ is smooth on $B^{\ell}\left(r_{1}+R, 0\right) \backslash\{0\}$. Since $\Psi$ is the identity map near $0, \Psi$ is smooth everywhere.

It follows from the above that $\Psi$ carries the standard radial vector field $\frac{\partial}{\partial r}$ on $B^{\ell}\left(r_{1}+R, 0\right) \backslash\{0\}$ to $-\eta_{\text {new }}^{F}$, which implies (1). Moreover, Lemma A.18 and the definition of $\eta_{\text {new }}^{F}$ imply that image $(\Psi)$ is exactly $F \backslash$ $\left(h^{F}\right)^{-1}((1-\varepsilon, 1])$. In particular, the boundary $S_{r_{1}+R}^{\ell-1}$ is carried to $Z_{\varepsilon}$.

We now show that $\Psi$ is a diffeomorphism. First, by the theory of ordinary differential equations, trajectories of $-\eta_{\text {new }}^{F}$ are disjoint, so $\Psi$ is injective. Next we claim that $\Psi$ is a local diffeomorphism for all $x \in B^{\ell}\left(r_{1}+\right.$ $R, 0)$. It suffices to prove that the differential $d \Psi_{x}$ is always onto. From the definition of $\Psi$, the claim is obvious for any point $x$ in the interior of $B^{\ell}\left(r_{1}, 0\right)$. Hence we may assume that $x \in B^{\ell}\left(r_{1}+R, 0\right)$ is of the form $x=$ $y+t_{0} \frac{y}{\|y\|}$ for $y \in S_{r_{1}}^{\ell-1}$ and $0 \leq t_{0}<R$. For a fixed $t$, denote by $\psi^{\text {new }}(t, \cdot)$ the map $x \mapsto \psi^{\text {new }}(t, x)$. By definition of $\Psi$, the image $(\Psi)_{*}\left(T_{x} S_{r_{1}+t_{0}}^{\ell-1}\right)$ is equal to $\left.\left(\psi^{\text {new }}\left(t_{0}, \cdot\right)\right)_{*}\left(T_{y} S_{r_{1}}^{\ell-1}\right)\right)$, and we have already seen above that $\Psi_{*}\left(\frac{\partial}{\partial r}\right)=$ $-\eta_{\text {new }}^{F}=\left(\psi^{\text {new }}\left(t_{0}, \cdot\right)\right)_{*}\left(\frac{\partial}{\partial r}\right)$. Since $\psi^{\text {new }}\left(t_{0}, \cdot\right)$ is a local diffeomorphism, we conclude $\Psi_{*}$ is also onto at every $x=y+t_{0} \frac{y}{\|y\|}$, as desired.

Since $\Psi$ is a local diffeomorphism, it is in particular an open map. Surjectivity now follows from the general fact that a continuous open map from a nonempty compact space to a connected Hausdorff space is surjective. Hence $\Psi$ is injective, surjective, and locally a diffeomorphism, hence a global diffeomorphism as desired.

We are now in a position to explicitly construct an extension of $f_{\ell-1}$ to the interior rel-int $F$ of $F$. We do so by using the reparameterization to $B\left(r_{1}+R, 0\right)$ given by $\Psi$. Recall that the function $f_{\ell-1}$ is defined on a neighborhood $U_{\ell-1}$ of $\partial F$ in $F$ that contains, by our assumption on $\varepsilon$, the hypersurface $Z_{\varepsilon}=\Psi\left(\partial B_{r_{1}+R}\right)$. Let $\Psi^{*} f_{\ell-1}$ be the pullback of $f_{\ell-1}$ to a neighborhood $U_{\partial B}$ of the boundary in $B\left(r_{1}+R, 0\right)$. Since

- $\Psi$ carries $-\frac{\partial}{\partial r}$ to $\eta_{\text {new }}^{F}$,

- $\eta_{\text {new }}^{F}$ coincides with $\eta^{F}$ near $Z_{\varepsilon}$, 
- $d f_{\ell-1}\left(\eta^{F}\right)<0$ near $Z_{\varepsilon}$, and

- $\alpha_{F}<\min _{Z_{\varepsilon}} f_{\ell-1}$,

after possibly shrinking the neighborhood $U_{\partial B}$ of $\partial B$ in $B\left(r_{1}+R, 0\right)$ we may assume that

$$
d\left(\Psi^{*} f_{\ell-1}\right)\left(-\frac{\partial}{\partial r}\right)<0 \text { on } U_{\partial B},
$$

and that there exists $\gamma \in \mathbb{R}$ such that $\alpha_{F}<\gamma<\inf _{U_{\partial B}} \Psi^{*} f_{\ell-1}$.

We now define a function which, when patched together with $\Psi^{*} f_{\ell-1}$ via a partition of unity, will yield the desired extension. Define

$$
\zeta(x):=\alpha_{F}+\left(\gamma-\alpha_{F}\right)\left(\frac{\|x\|^{2}}{\left|r_{1}+R\right|^{2}}\right) \quad \text { for } x \in B\left(r_{1}+R, 0\right) \subseteq \mathbb{R}^{\ell}
$$

Then by construction $\zeta(x)<\gamma$ for all $x \in B\left(r_{1}+R, 0\right)$, so

$$
\Psi^{*} f_{\ell-1}>\zeta \text { on } U_{\partial B}
$$

Since $\partial B\left(r_{1}+R, 0\right)$ is compact, there exists an $\tilde{R}$ with $0<\tilde{R}<r_{1}+R$ such that the annulus

$$
\left\{x \mid \tilde{R} \leq\|x\| \leq r_{1}+R\right\}
$$

is contained in the neighborhood $U_{\partial B}$ of $\partial B\left(r_{1}+R, 0\right)$. Let $\bar{\rho}:\left[0, r_{1}+R\right] \rightarrow$ $\mathbb{R}$ be a smooth function such that

- $\bar{\rho}$ is weakly monotone increasing, and

- there exist $R_{1}$ and $R_{2}$ such that $0<\tilde{R}<R_{1}<R_{2}<r_{1}+R$ and such that $\bar{\rho}=0$ on $\left[0, R_{1}\right]$ and $\bar{\rho}=1$ on $\left[R_{2}, r_{1}+R\right]$.

Given such a $\bar{\rho}$, define $\rho: B\left(r_{1}+R, 0\right) \rightarrow \mathbb{R}$ by $\rho(x):=\bar{\rho}(\|x\|)$. Note that $\left(-\frac{\partial}{\partial r}\right) \rho \leq 0$ by assumption on $\bar{\rho}$. Consider the function

$$
\rho \Psi^{*} f_{\ell-1}+(1-\rho) \zeta: B^{\ell}\left(r_{1}+R, 0\right) \rightarrow \mathbb{R}
$$

Lemma A.24. The function (A.23)

- is smooth;

- has a unique critical point in the relative interior $\stackrel{\circ}{B}_{r_{1}+R}$, and this critical point is at the origin 0 ;

- at 0 it takes the value $\alpha_{F}$. 
Moreover, there exists a neighborhood of $\partial B\left(r_{1}+R, 0\right)$ in $\mathbb{R}^{\ell}$ on which this function agrees with $\Psi^{*} f_{\ell-1}$.

Proof. The smoothness of (A.23) follows immediately from the fact that it is a smooth convex sum of two smooth functions. To show that the only critical point is at 0 , suppose that $x \neq 0$. We will show that the differential does not vanish at $x$. We compute

$$
\begin{aligned}
-\frac{\partial}{\partial r}\left(\rho \Psi^{*} f_{\ell-1}+(1-\rho) \zeta\right)= & \rho\left(-\frac{\partial}{\partial r} \Psi^{*} f_{\ell-1}\right)+(1-\rho)\left(-\frac{\partial}{\partial r} \zeta\right) \\
& -\frac{d \rho}{d r} \Psi^{*} f_{\ell-1}-\frac{d(1-\rho)}{d r} \zeta \\
= & \rho\left(-\frac{\partial}{\partial r} \Psi^{*} f_{\ell-1}\right)+(1-\rho)\left(-\frac{\partial}{\partial r} \zeta\right) \\
& -\frac{d \rho}{d r}\left(\Psi^{*} f_{\ell-1}-\zeta\right) .
\end{aligned}
$$

Since $-\frac{\partial}{\partial r} \Psi^{*} f_{\ell-1}<0$ where defined (i.e., near the boundary), $-\frac{\partial}{\partial r} \zeta<0$ where defined and for $x \neq 0, \quad \Psi^{*} f_{\ell-1}-\zeta>0$ by construction of $\zeta$, and $-\frac{\partial}{\partial r} \rho \leq 0$ by assumption, the last quantity is always $\leq 0$. In fact, by the above, at least one of the first two terms must be strictly negative for any $x \neq 0$. Hence the quantity is non-zero and we conclude that the points $x \neq 0$ are not critical points of (A.23). On the other hand, for $x=0$, since $\zeta$ is defined in terms of the norm-square $\|x\|^{2}$, it is immediate that $x=0$ is a critical point. Finally, in the neighborhood of $\partial B r_{1}+R$ where $\rho \equiv 1$ the function (A.23) is equal to $\Psi^{*} f_{\ell-1}$.

Now consider the pullback of the function (A.23) to $F \backslash\left(h^{F}\right)^{-1}((1-$ $\varepsilon, 1])$ via the diffeomorphism $\Psi^{-1}$ inverse to $\Psi$. By Lemma A.24, this pullback agrees with $f_{\ell-1}$ on a neighborhood of the boundary $Z_{\varepsilon}$. Thus we may extend this pullback to a smooth function $f_{\ell, F}$ on all of $F$ by setting $f_{\ell, F}:=f_{\ell-1}$ on $\left(h^{F}\right)^{-1}((1-\varepsilon, 1])$. This function $f_{\ell, F}$ has the properties that

- $f_{\ell, F}$ has a unique critical point at the origin $0 \in F$;

- the unique critical point 0 is a global minimum; and

- $f_{\ell, F}(0)=\alpha_{F}$.

We have achieved our goal of extending the initial function $f_{\ell-1}$ (after possibly shrinking its domain of definition) to a function $f_{\ell}$ that is defined on the entire face $F$. Repeating this for every $\ell$ dimensional face, we obtain a function $f_{\ell, F}$ on each $\ell$ dimensional face $F$ such that $F_{\ell, F}$ agrees with 
$f_{\ell-1}$ on some neighborhood $U_{F}$ of $\partial F$ in $F$. We would like to extend these functions further to a whole ( $n$-dimensional) neighborhood of the $\ell$-skeleton of $\Delta$ whole ensuring that the required conditions (f1)-(f3) continue to hold.

Below, we extend each $f_{\ell, F}$ to a smooth function $\tilde{f}_{\ell, F}$ on a neighborhood of $F$ in $\Delta$ such that each function $\tilde{f}_{\ell, F}$ agrees with $f_{\ell-1}$ on a neighborhood of $\partial F$ in $\Delta$ and satisfies the derivative condition

$$
d\left(\tilde{f}_{\ell, F}\right)\left(\xi_{j}\right)<0
$$

on $F$ for all $j$ such that $F \subset \sigma_{j}$. Before proceeding, and supposing for a moment that such functions $\tilde{f}_{\ell, F}$ can be constructed, we first explain how this completes the proof of the statements (A) and (B) of Proposition A.1. Choose open subsets $V_{F}$ of $\Delta$ such that $F \subseteq V_{F}$ and $V_{F} \subseteq U_{F}$. By shrinking the $V_{F}$ if necessary, we may without loss of generality assume that for two distinct nontrivially intersecting faces $F \cap F^{\prime} \neq \emptyset$, the functions $\tilde{f}_{\ell, F}$ and $\tilde{f}_{\ell, F^{\prime}}$ agree on the overlap of the open sets, i.e., $\left.\tilde{f}_{\ell, F}\right|_{V_{F} \cap V_{F^{\prime}}}=\left.\tilde{f}_{\ell, F^{\prime}}\right|_{V_{F} \cap V_{F^{\prime}}}$, since the $\left\{\tilde{f}_{\ell, F}\right\}$ are assumed to agree on a neighborhood in $\Delta$ of the $(\ell-1)$ skeleton (which contains $F \cap F^{\prime}$ ). With this understood, we may therefore define a smooth function $f_{\ell}$ on the open neighborhood $\bigcup_{F} V_{F}$ in $\Delta$ of the $\ell$-skeleton by $\left.f_{\ell}\right|_{V_{F}}:=\tilde{f}_{\ell, F}$. By construction, $f_{\ell}$ satisfies the properties (f1)(f3) listed above. This then completes the inductive step and hence the proof.

Hence, to complete the proof of statements (A) and (B) of Proposition A.1, it remains only to construct these extensions $\tilde{f}_{\ell, F}$. We begin by choosing a convenient (non-linear) coordinate chart. Recall that we are assuming that the affine span of the face $F$ is $\mathbb{R}^{\ell}$, embedded in $\mathbb{R}^{n}$ as $\mathbb{R}^{\ell} \times\{0\}^{n-\ell}$. Fix smooth extensions of the vector fields $\xi_{1}, \ldots, \xi_{n-\ell}$ to $\mathbb{R}^{\ell}$. By Lemma A.3, the vectors $\left.\xi_{1}\right|_{x}, \ldots,\left.\xi_{n-\ell}\right|_{x}$ are linearly independent and span a complementary subspace to $\mathbb{R}^{\ell} \subset \mathbb{R}^{n}$ at each point $x \in F$. Thus, the differential of the map

$$
\begin{aligned}
\varphi: \mathbb{R}^{\ell} \times \mathbb{R}^{n-\ell} & \rightarrow \mathbb{R}^{n} \\
\left(x, y_{1}, \ldots, y_{n-\ell}\right) & \mapsto x+\left.y_{1} \xi_{1}\right|_{x}+\cdots+\left.y_{n-\ell} \xi_{n-\ell}\right|_{x}
\end{aligned}
$$

is a linear isomorphism at each point $x$ of $F$. This implies that there exists $\varepsilon>0$ and a neighborhood $W_{F}$ of $F$ in $\mathbb{R}^{\ell}$ such that the map $\varphi$ restricts to a diffeomorphism of $W_{F} \times(-\varepsilon, \varepsilon)^{n-\ell}$ with an open subset of $\mathbb{R}^{n}$ that carries a neighborhood of $F \times\{0\}^{n-\ell}$ in $F \times \mathbb{R}_{>0}^{n-\ell}$ to a neighborhood of $F$ in $\Delta$. In the argument below, we therefore use these coordinates $\left(x, y_{1}, \ldots, y_{n-\ell}\right) \in$ $F \times(-\varepsilon, \varepsilon)^{n-\ell} \subseteq F \times \mathbb{R}^{n-\ell}$ to parameterize a neighborhood of $F$ in $\mathbb{R}^{n}$. 
By assumption on $f_{\ell-1}$, there exists some $0<\varepsilon^{\prime}<\varepsilon$ and a neighborhood $W_{1}$ of $\partial F$ in $F$ such that $\varphi^{*} f_{\ell-1}$ is defined on $W_{\partial F}:=W_{1} \times\left(-\varepsilon^{\prime}, \varepsilon^{\prime}\right)^{n-\ell}$. Let $W_{2}$ be the relative interior of $F$ in $\mathbb{R}^{\ell}$. Then $\left\{W_{1}, W_{2}\right\}$ form an open cover of $F$. Let $\left\{\rho_{1}, \rho_{2}\right\}$ be a partition of unity subordinate to this cover. For $(x, y) \in F \times\left(-\varepsilon^{\prime}, \varepsilon^{\prime}\right)^{n-\ell}$, we define

(A.26) $\quad \tilde{f}_{\ell, F}(\varphi(x, y)):=\rho_{1}(x) \varphi^{*} f_{\ell-1}(x, y)+\rho_{2}(x)\left(\varphi^{*} f_{\ell, F}(x)-\sum_{i=1}^{n-\ell} y_{i}\right)$.

Since $\rho_{2}$ is supported in $W_{2}$, there exists a neighborhood of $\partial F$ in $\mathbb{R}^{\ell}$ on which $\rho_{2} \equiv 0$. This means that on a neighborhood in $\mathbb{R}^{n}$ of the $(\ell-1)$-skeleton near $F, \quad \tilde{f}_{\ell, F} \equiv f_{\ell-1}$, as desired. The only remaining claim needing proof is that $\tilde{f}_{\ell, F}$ satisfies the derivative condition $d\left(\tilde{f}_{\ell, F}\right)\left(\xi_{j}\right)<0$ on $F$ for $1 \leq j \leq n-\ell$. Since $\tilde{f}_{\ell, F}$ agrees with $f_{\ell-1}$ on a neighborhood of $\partial F$, it suffices to check this condition on rel-int $(F)$. Since $\varphi_{*}\left(\frac{\partial}{\partial y_{j}}\right)=\xi_{j}$ by construction of $\varphi$, and the three functions $\rho_{1}, \rho_{2}$, and $f_{\ell, F}$ are independent of the $y_{j}$ variables, we have

$$
\begin{aligned}
d\left(\tilde{f}_{\ell, F}\right)\left(\left.\xi_{j}\right|_{\varphi(x, 0)}\right) & =\left.\frac{\partial}{\partial y_{j}}\right|_{(x, 0)}\left[\rho_{1}(x)\left(\varphi^{*} f_{\ell-1}\right)(x, y)+\rho_{2}(x)\left(f_{\ell, F}(x)-\sum_{i=1}^{n-\ell} y_{i}\right)\right] \\
& =\rho_{1}(x)\left(d f_{\ell-1}\right)\left(\left.\xi_{j}\right|_{x}\right)-\rho_{2}(x) \\
& <0
\end{aligned}
$$

as desired, since $\left(d f_{\ell-1}\left(\left.\xi_{j}\right|_{x}\right)<0\right.$ by assumption and at least one of $\rho_{1}$ or $\rho_{2}$ must be positive at any $x \in F$. This completes the proof of the claim and hence of the statements (A) and (B) in Proposition A.1.

It remains to justify the statement (C) of Proposition A.1. In a small enough neighborhood of the prescribed critical point $x_{F}$ of a face $F$, the function defined in (A.23) has the property that $\rho \equiv 0$ and hence, along the face $F$, is equal to $\zeta$. The explicit formula for $\zeta$ in (A.22) shows that, in appropriate coordinates along the face $F$, the function $\zeta$ is quadratic in the coordinates up to an affine translation in $\mathbb{R}$, as desired. Moreover, the explicit formula for $\tilde{f}_{\ell, F}$ in (A.26) shows that with respect to the coordinates $(x, y)$ in (A.25) is linear in the coordinates $y_{j}$ and decreases in the directions pointing into the polytope $F$, again as desired. Since the vector fields $\xi_{j}$ are also arranged to be constant sufficiently near $x_{F}$, the coordinates $(x, y)$ are in fact affine. This concludes the proof of part (C) of Proposition A.1 and hence of the entire proposition. 


\section{References}

[1] J. Agapito and L. Godinho, New polytope decompositions and EulerMaclaurin formulas for simple integral polytopes, Adv. Math. 214(1) (2007), 379-416.

[2] M.F. Atiyah and R. Bott, The moment map and equivariant cohomology, Topology 23 (1984), 1-28.

[3] N. Berline and M. Vergne, Classes caractéristiques équivariantes. Formule de localisation en cohomologie équivariante, C. R. Acad. Sci. Paris Sér. I Math. 295 (1982), 539-541.

[4] N. Berline and M. Vergne, Zéros d'un champs de vecteurs et classes caractéristiques équivariantes, Duke Math. J. 50 (1983), 539-549.

[5] R. Bott, Vector fields and characteristic numbers, Mich. Math. J. 14 (1967), 231-244.

[6] R. Bott, A residue formula for holomorphic vector fields, J. Differential Geom. 1 (1967), 311-330.

[7] M. Braverman, Index theorem for equivariant Dirac operators on noncompact manifolds, K-Theory 27(1) (2002), 61-101.

[8] C.J. Brianchon, Théorème nouveau sur les polyèdres, J. Ecole Polytechnique, 15 (1837), 317-319.

[9] Th. Bröcker and K. Jänich, Introduction to differential topology, Cambridge University Press, 1982.

[10] J. Carrell, A remark on the Grothendieck residue map, Proc. Amer. Math. Soc. 70(1) (1978), 43-48.

[11] A. Cannas da Silva and V. Guillemin, On the Kostant multiplicity formula for group actions with non-isolated fixed points, Adv. Math. 123(1) (1996), 1-15.

[12] A. Cannas da Silva, Lectures on symplectic geometry, Springer-Verlag, Lecture Notes in Mathematics, 2001.

[13] J.J. Duistermaat and G.J. Heckman. On the variation in the cohomology of the symplectic form of the reduced phase space, Invent. Math. 69(2) (1982), 259-268. 
[14] J.J. Duistermaat and G.J. Heckman. Addendum to: On the variation in the cohomology of the symplectic form of the reduced phase space, Invent. Math. 72(1) (1983), 153-158.

[15] V. Ginzburg, V. Guillemin and Y. Karshon, Cobordism theory and localization formulas for Hamiltonian group actions, Int. Math. Res. Notices 5 (1996), 221-234.

[16] V. Ginzburg, V. Guillemin and Y. Karshon, Moment maps, Cobordisms, and Hamiltonian group actions, Amer. Math. Soc., Math. Surveys and Monographs vol. 98, Providence, RI, 2002.

[17] J.P. Gram, Om rumvinklerne $i$ et polyeder, Tidsskrift for Math. (Copenhagen) 4(3) (1874), 161-163.

[18] V. Guillemin, E. Lerman and S. Sternberg, Symplectic fibrations and multiplicity diagrams, Cambridge University Press, 1994.

[19] V. Guillemin and A. Pollack, Differential topology, Prentice-Hall, 1974.

[20] V. Guillemin and S. Sternberg, Convexity properties of the moment mapping, Invent. Math. 67 (1982), 491-513.

[21] V. Guillemin and S. Sternberg, Cohomological properties of the moment mapping, unpublished preprint 1982.

[22] C. Haase, Polar decomposition and Brion's theorem, in Integer points in polyhedra - geometry, number theory, algebra, optimization, volume 374 of Contemp. Math., 91-99. Amer. Math. Soc., Providence, RI, 2005.

[23] A. Hatcher, Algebraic topology, Cambridge University Press, 2002.

[24] L. Jeffrey and F. Kirwan, Localization for nonabelian group actions, Topology 34(2) (1995), 291-327.

[25] Y. Karshon, Moment maps and non-compact cobordisms, J. Differential Geometry 49 (1998), 183-201.

[26] J. Lawrence, Polytope volume computation, Math. Comp. 57(195) (1991), 259-271.

[27] K. Liu, Remarks on nonabelian localization, Int. Math. Res. Notices 13 (1995), 683-691.

[28] S. Martin, Transversality theory, cobordisms, and invariants of symplectic quotients, arXiv:math.SG/0001001. 
[29] P.-E. Paradan, The moment map and equivariant cohomology with generalized coefficients, Topology 39(2) (2000), 401-444.

[30] E. Prato and S. Wu, Duistermaat-Heckman measures in a noncompact setting, Composit. Math. 94(2) (1994), 113-128.

[31] G.C. Shephard, An elementary proof of Grams theorem for convex polytopes, Can. J. Math. 19 (1967), 1214-1217.

[32] A.N. Varchenko, Combinatorics and topology of the arrangement of ane hyperplanes in the real space, Funktsional. Anal. i Prilozhen. 21(1) (1987), 11-22.

[33] E. Witten, Two dimensional gauge theories revisited, J. Geom. Phys. 9 (1992), 303-368.

[34] C.T. Woodward, Localization for the norm-square of the moment map and the two-dimensional Yang-Mills integral, J. Symplectic Geom. 3(1) (2005), 17-54.

Department of Mathematics and Statistics

MCMASTER UNIVERSITY

HAMILTON

Ontario L8S 4K1, CANAda

E-mail address: Megumi.Harada@math.mcmaster.ca

URL: http://www.math.mcmaster.ca/Megumi.Harada/

Department of Mathematics

UNIVERSITY OF TORONTO

Toronto Ontario M5S 2E4, CAnAdA

E-mail address: karshon@math.toronto.edu

URL: http://www.math.toronto.edu/karshon

RECEIVED JANUARY 27, 2011 
